\title{
The Laser Alignment System for the CMS Silicon Microstrip Tracker
}

Von der Fakultät für Mathematik, Informatik und Naturwissenschaften der

Rheinisch-Westfälischen Technischen Hochschule Aachen zur Erlangung des akademischen Grades eines Doktors der Naturwissenschaften genehmigte Dissertation

vorgelegt von

Diplom-Physiker

Bruno Wittmer

aus Lissabon, Portugal

Berichter: Universitätsprofessor Dr. Stefan Schael

Universitätsprofessor Dr. Klaus Lübelsmeyer

Tag der mündlichen Prüfung: 13. Februar 2002

Diese Dissertation ist auf den Internetseiten der Hochschulbibliothek online verfügbar. 


\section{Contents}

1 Introduction 3

1.1 The Search for the Higgs Boson . . . . . . . . . . . . . . . . 3

1.2 The LHC Collider . . . . . . . . . . . . . . . . . . . . 3

1.2.1 Design Parasmeters . . . . . . . . . . . . . . . . . 3

1.2.2 Higgs Production at the LHC . . . . . . . . . . . . 4

1.3 The CMS Experiment . . . . . . . . . . . . . . . . 5

1.3.1 Physics goals of CMS . . . . . . . . . . . . . . . . 5

1.3.2 The CMS Detector . . . . . . . . . . . . . . . 6

1.4 The Inner Tracker . . . . . . . . . . . . . . . . . . . . . . . . . . . . . . . 9

1.4.1 Specifications and layout . . . . . . . . . . . . . 9 9

1.4.2 The silicon strip detectors . . . . . . . . . . . . . . . . 12

1.4.3 The Mechanical Support Structure . . . . . . . . . . . . 15

1.5 Purpose of the Alignment System . . . . . . . . . . . . . 18

1.6 Working Principle of the Alignment System _ . . . . . . . . . 19

2 Design Considerations for the Alignment System 21

2.1 Light Sources . . . . . . . . . . . . . . . . . . . . . . 21

2.2 Transparent Detectors . . . . . . . . . . . . . . . . 26

2.3 Transmission and Absorption of the laser beam . . . . . . . . 27

2.3.1 Absorption in silicon . . . . . . . . . . . . . . 27

2.3.2 Reflection at silicon-air interface . . . . . . . . . . . . 28

2.3 .3 Measurement of Transmission . . . . . . . . . . . . . . . . . . . . 29

2.3.4 Interference Effects . . . . . . . . . . . . . . . 30

2.4 Refraction of the beam . . . . . . . . . . . . . . . . 34

2.5 Accuracy in determining the laser spot position . . . . . . . . 36

2.5.1 Accuracy of the fit . . . . . . . . . . . . 37

2.5.2 Using statistics to improve $\mathrm{S} / \mathrm{N}$ ratio $\ldots \ldots \ldots$

2.5.3 Effects of distorted profiles . . . . . . . . . . . . . . 40

3 The CMS Laser Alignment System 48

3.1 Layout . . . . . . . . . . . . . . . . . . . . . 48

3.1.1 Number and Position of Beams . . . . . . . . . . . . . 48

3.1.2 Nd-YAG Laser / Fiber Coupling . . . . . . . . . . . . 50

3.2 Performance Parameters . . . . . . . . . . . . . . . . . . 51 
3.2.1 Refraction ...................... 51

3.2.2 Laser spot accuracy . . . . . . . . . . . . . . . . . . 52

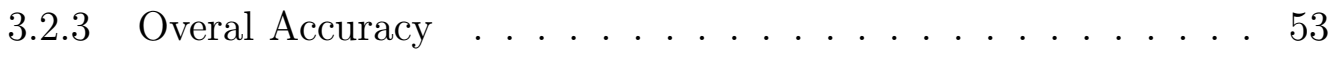

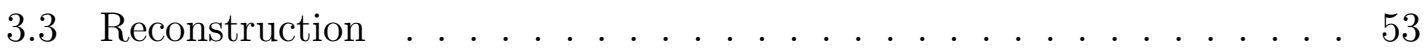

3.3.1 Layout and Parametrisation . . . . . . . . . . . . 54

3.3 .2 Symmetries . . . . . . . . . . . . . . . 57

3.3.3 Error function and minimization . . . . . . . . . . . . 57

3.4 Simulation of the Performance . . . . . . . . . . . . . . . 59

3.4.1 Simulation Principle . . . . . . . . . . . . . . . . 59

3.4.2 Results of the simulation . . . . . . . . . . . . . 59

4 Conclusion $\quad 65$

4.1 Summary ............................. 65

4.2 Open Issues . . . . . . . . . . . . . . . . . . . . 66

A Accuracy in determining the laser spot on the module $\quad 69$

$\begin{array}{ll}\text { B Equations used for the reconstruction } & 71\end{array}$ 


\section{Chapter 1}

\section{Introduction}

\subsection{The Search for the Higgs Boson}

In the last few decades, the standard model of particle physics (SM) has been confirmed in many experiments at various colliders. One of the last missing pieces in the standard model is the Higgs Boson, which was introduced to give rise to the different particle masses. In the minimal SM the Higgs Boson is a neutral scalar particle.

The search for the Higgs Boson has so far been unsuccessful, except for a few candidate events found at LEP in its last months of data taking. An excess of 2 standard deviations above the expected background was found [1]. Figure 1.1 shows the log-likelihood ratio for the measured data, the expected background and the expected signal+background as a function of the Higgs mass. The fourth curve is a test, where the signal of a $115 \mathrm{GeV}$ Higgs Boson was added to the background and propagated through the likelihood calculation. The obseved events suggest that there might be a Higgs particle with a mass around $115 \mathrm{GeV}$. The data also allows to set a lower bound of $114.1 \mathrm{GeV}$ for the Higgs mass $m_{H}$. Upper limits for $m_{H}$ come from precision measurements of electroweak parameters, yielding an upper bound of $196 \mathrm{GeV}$ for $m_{H}$ with a $95 \%$ confidence level.

\subsection{The LHC Collider}

\subsubsection{Design Parasmeters}

To find clear evidence for the Higgs Boson and gather enough statistics to determine its mass and width, accelerators with enough center of mass energy are needed. For this purpose, as well as for the search of physics beyond the standard model, the Large Hadron Collider (LHC) will be built at CERN, and is scheduled to start running in 2005 [3].

The LHC will collide protons accelerated to an energy of $7 \mathrm{TeV}$, resulting in a center of mass energy $\sqrt{s}$ of $14 \mathrm{TeV}$. Since the proton consists of many quarks 


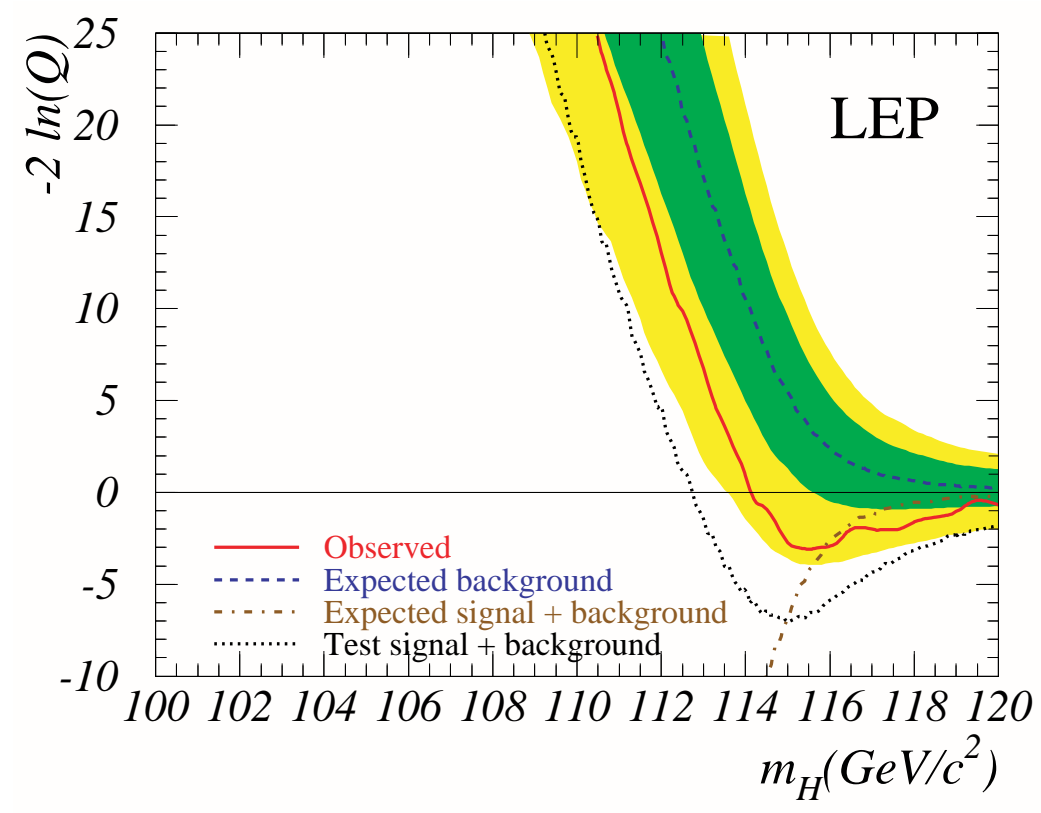

Figure 1.1: Log-likelihood ratio as function of the Higgs mass [2]. The shaded regions denominate the $1 \sigma$ and $2 \sigma$ range around the background.

and gluons, only a fraction of $\sqrt{s}$ will participate in a p-p interaction, so that only particles with masses up to about $1 \mathrm{TeV}$ may be produced in the collisions.

The luminosity of the LHC collider will be staged in two phases. During the first years of operation the collider will run at one 10th of the design luminosity which is $10^{34} \mathrm{~cm}^{-2} \mathrm{~s}^{-1}$. At the final luminosity the bunches will collide at a rate of $40 \mathrm{MHz}$ with $10^{11}$ protons per bunch. In average there will be some 20 interactions per bunch crossing spread over a region with an extent of $7.5 \mathrm{~cm}$ rms along the beam direction.

Apart from protons the LHC will also be able to accelerate and collide lead ions and other heavy nuclei at energies up to $1000 \mathrm{TeV}$. These heavy ion collisions will allow to study the formation of quark-gluon plasma.

Four experiments will be installed at the LHC. Two of them (CMS and ATLAS) are general purpose detectors, while LHCb and ALICE are dedicated to b-physics and heavy ion collisions respectively. In section 1.3 the CMS experiment will be described in more detail.

\subsubsection{Higgs Production at the LHC}

At the LHC the Higgs Boson will be produced in several different processes $[4,5,6,7]$. Figure 1.2 shows the crossections for the most relevant production mechanisms as function of $m_{H}$. As can be seen they are dominated by the gluon-gluon fusion where the crossection almost becomes $100 \mathrm{pb}$ at Higgs masses of $115 \mathrm{GeV}$. The crossection falls quickly with increasing Higgs mass. At masses near $1 \mathrm{TeV}$ the production 
via vector boson fusion ( $\mathrm{qq} \rightarrow \mathrm{Hqq}$ ) becomes comparable to the gluon-gluon fusion. The crossection for Higgsstrahlung and bremsstrahlung off top quarks only becomes sizeable for Higgs masses smaller than $100 \mathrm{GeV}$ which have already been ruled out by the LEP searches.

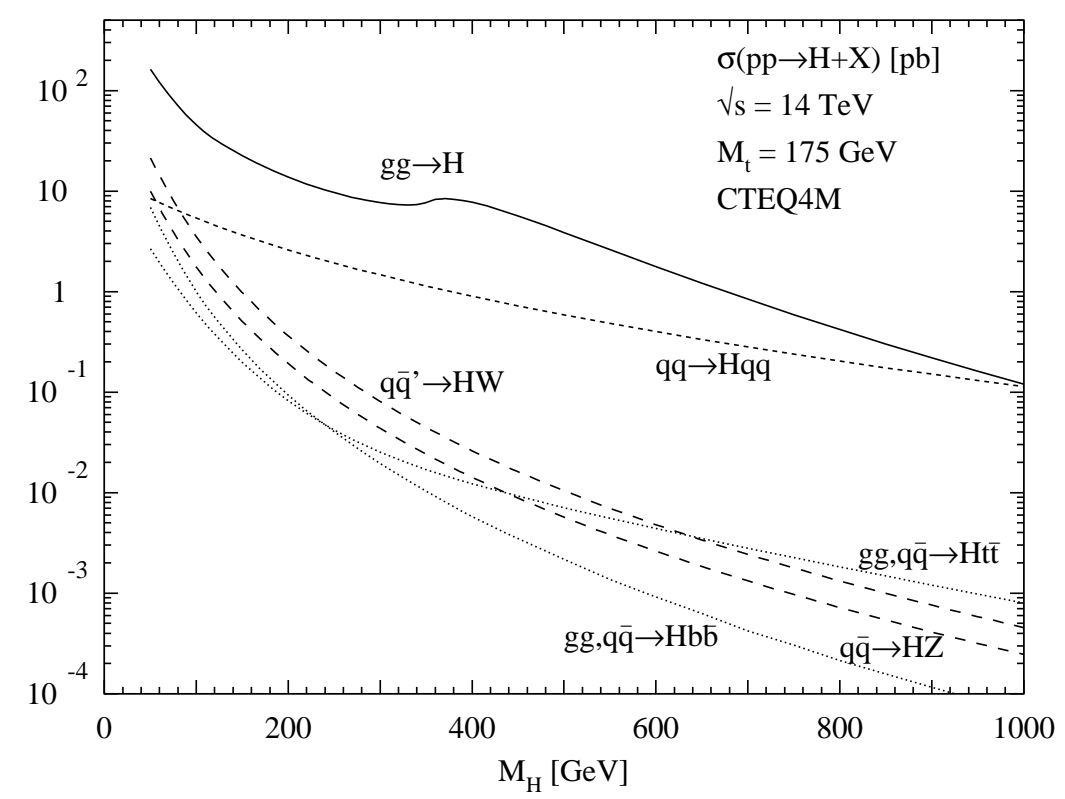

Figure 1.2: Crossections for the Higgs production at LHC as function of the Higgs mass $m_{H}[4]$.

\subsection{The CMS Experiment}

\subsubsection{Physics goals of CMS}

The Compact Muon Solenoid (CMS) experiment has been designed to find clear signatures of the Higgs boson in a large mass range, to search for new physics, e.g. supersymmetric particles, perform studies of the physics of bottom (b) and top ( $t$ ) quarks and study heavy ion collisions [8].

Depending on the Higgs mass, there are several decay channels that can be used for the Higgs search. Figure 1.3 shows the branching ratio for the different decay channels as function of $m_{H}$. At low masses, the two photon decay will allow the Higgs discovery for masses up to $150 \mathrm{GeV}$. Although the branching ratio for this process is very small, its background is small enough and well known, making it suitable for Higgs searches. For this channel an electromagnetic calorimeter with high granularity and good energy resolution is needed. At higher masses, the decays of the Higgs into a $\mathrm{W}$ or $\mathrm{Z}$ pair with four leptons or two leptons and two jets in the final state become more important. CMS must therefore be able to measure 
tracks of muons and electrons with high transverse momentum $p_{t}$, reconstruct jets with high efficiency and detect missing energy in the case that a neutrino is present in the final state. A tracking system with good momentum resolution, an efficient muon identification and good calorimetry are the prerequisites for this search.

Missing energy and high energy jets are also the signatures for the presence of supersymmetric particles. For the b- and t-physics a high vertex resolution is necessary to allow a good measurement of the impact parameter needed for tagging. For the study of quark-gluon plasma in the case of heavy ion collisions, the reconstruction of $\Upsilon$ mesons is needed. For this purpose a good tracking system is needed, with the possibility to reconstruct low momentum muons. All these requirements lead to the design of a very flexible general purpose detector.

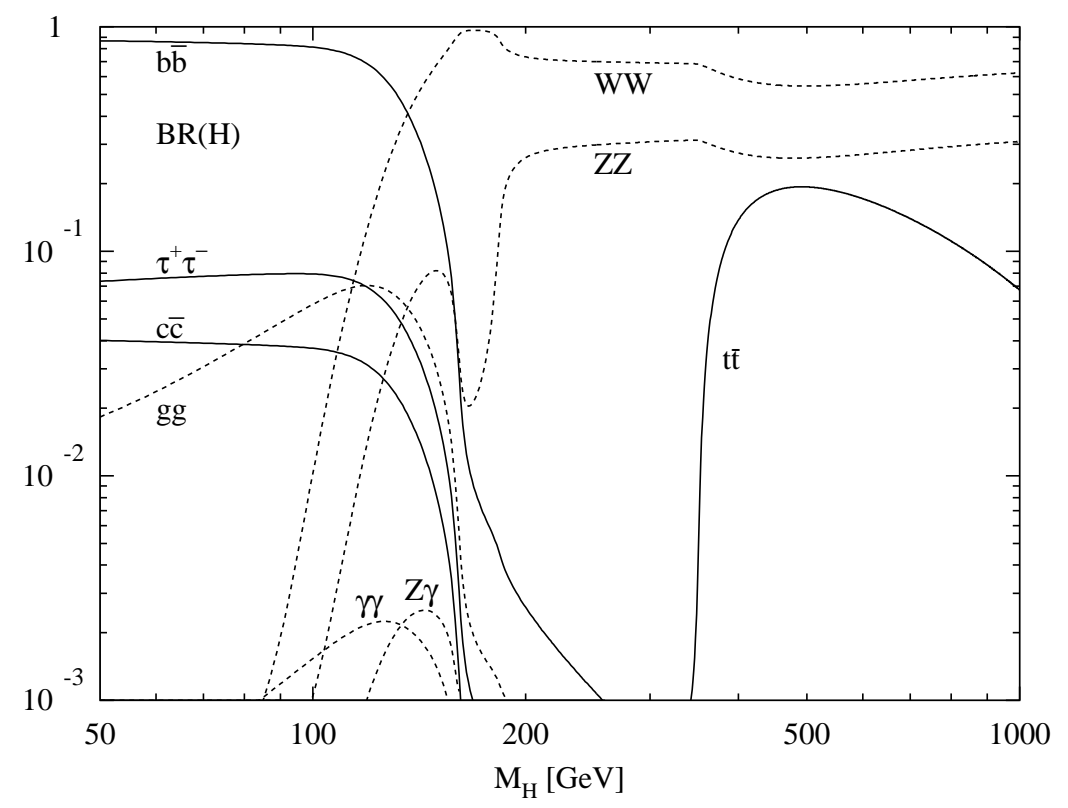

Figure 1.3: Branching ratios for the different decay channels of the Higgs boson [4].

\subsubsection{The CMS Detector}

The CMS detector consists of several subdetectors arranged inside and around a solenoid magnet (fig.1.4). The superconducting coil generates a field of $4 \mathrm{~T}$ in its inner region and around $2 \mathrm{~T}$ in the return yoke [9]. This allows a very good momentum resolution when measuring charged particle tracks with high transversal momentum $p_{t}$. The strong magnetic field also confines tracks of low momentum particles to the forward direction, which helps to get a robust trigger on muons with high $p_{t}$. Finally the $4 \mathrm{~T}$ field allows an efficient calibration of the electromagnetic calorimeter with electron tracks.

The return yoke of the magnet is instrumented with muon detectors, consisting of drift tubes, cathode strip chambers and resistive plate chambers [10]. The drift 
tubes are installed in the barrel region where they allow a very ggod measurement of the momentum of high $p_{t}$ muons. In the two endcaps cathode strip chambers are used for the momentum measurement, which are better suited for the operation in a varying magnetic field. They are also faster and have a smaller segmentation than the drift tubes, which is important for the higher particle rate in the forward direction. The resistive plate chambers are very fast and highly segmented sensors, which are used for triggering and bunch cross identification. The muon system identifies the muons and measures their momentum with sufficient resolution $(\approx$ $12 \% \delta p_{t} / p_{t}$ at $10 \mathrm{GeV}$ and $30 \% \delta p_{t} / p_{t}$ at $\left.1 \mathrm{TeV}\right)$ to allow triggering at various thresholds.

Inside the magnet coil are the hadronic calorimeter (HCAL), the electromagnetic calorimeter (ECAL) and the tracker. The task of the calorimeters is to measure the direction and the energy of jets and of isolated electrons or photons. They also measure missing energy flow which is an indication for the presence of neutrinos or new particles like the supersymmetric partners of quarks. Furthermore they allow particle identification in conjunction with the muon system and the tracker. The calorimeters are placed inside the magnet coil to minimize the material that is crossed by the particles before their energy is measured. In this way the energy resolution can be optimized.

The HCAL is a sampling calorimeter made of copper absorber plates and plastic scintillators [11]. Its thickness at $\eta=0$ is $79 \mathrm{~cm}$ which corresponds to 5.15 hadronic interaction lengths. To cover high rapidity ranges $(3<\eta<5)$, additional forward hadronic calorimeters are placed outside the muon system. The active components of these forward calorimeters are quartz fibres, which are very radiation hard.

The ECAL is made of $\mathrm{PbWO}_{4}$ crystals, which provide a good energy resolution and are radiation hard at the same time [12]. They also have a short radiation length and a small Molière radius. In the barrel the crystals are $23 \mathrm{~cm}$ long, which corresponds to 26 radiation lengths. In the endcaps the crystals can be slightly shorter, since they are preceeded by a preshower detector containing three radiation lengths of lead. In the barrel region the crystals are read out by avalanche photodiodes (APD's) which can be operated at a high transverse magnetic field. In the endcaps the high radiation levels impose the use of a different technology. Here the crystals are read out by vacuum phototriodes (VPT's). The overall energy resolution $\sigma_{E} / E$ of the ECAL is around $2 \%$ at $10 \mathrm{GeV}$ going down to $0.55 \%$ at $1 \mathrm{TeV}$.

The innermost part of the CMS detector is the tracker which will be described in detail in the next section. 


\section{C.M.S. \\ A Compact Solenoidal Detector for L.H.C.}

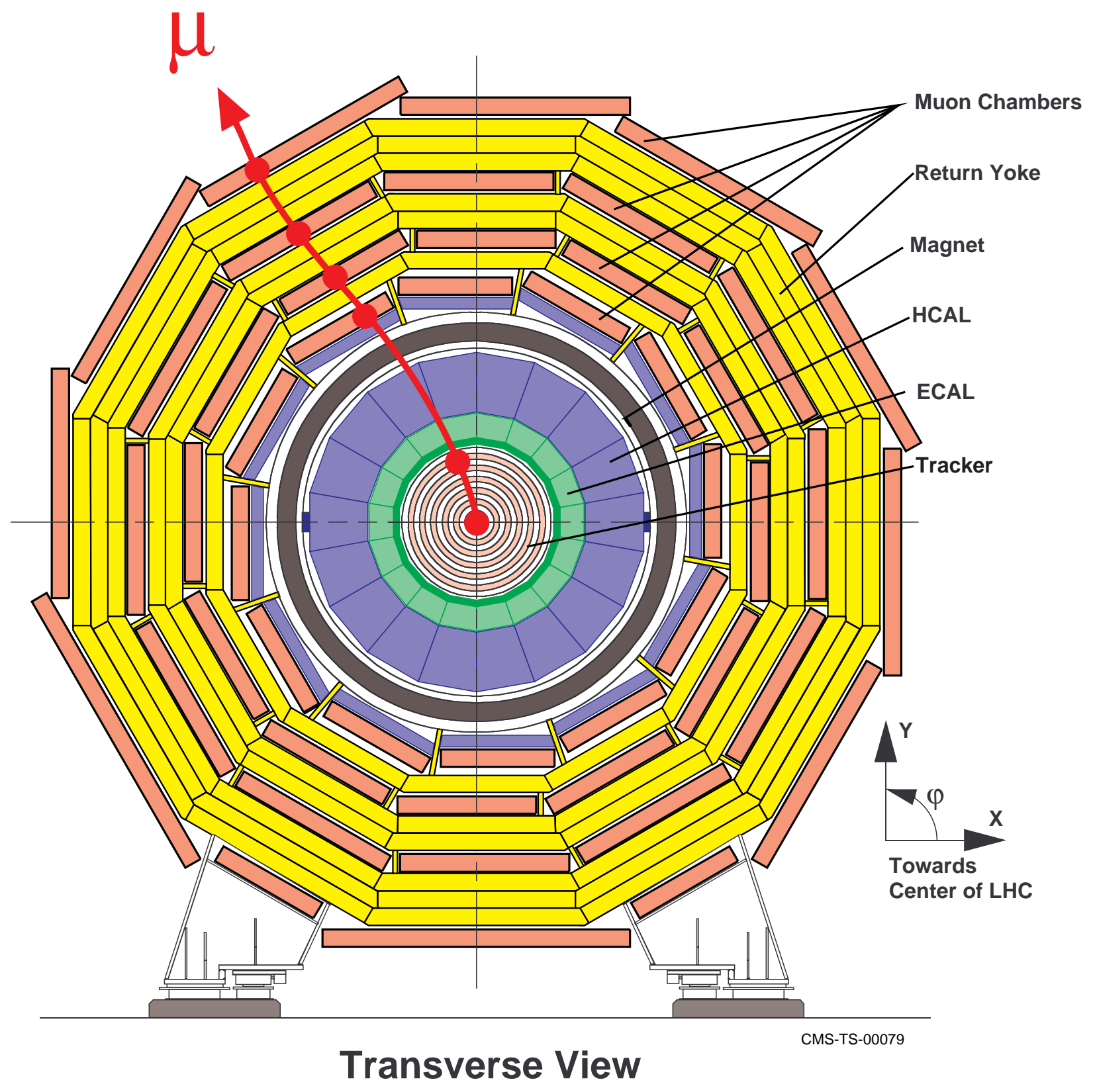

Figure 1.4: Transverse view of the CMS detector. 


\subsection{The Inner Tracker}

\subsubsection{Specifications and layout}

The CMS tracker has been designed to allow effective pattern recognition for tracks of charged particles, good vertex resolution and precise momentum measurement [13, 14]. It is organized in several subsystems. In the innermost region there are 2 layers of pixel detectors, which have a very high granularity and provide 3-dimensional hit information. They will be used to measure impact parameters for the b-tagging and to simplify the pattern recognition task for the track reconstruction.

The layout of the silicon microstrip tracker is shown in fig.1.5. ${ }^{1}$ It extends from a radius of $200 \mathrm{~mm}$ up to $1100 \mathrm{~mm}$, and is divided into a barrel region and two endcaps. Each endcap (TEC) has 9 disks equipped with up to 7 rings of wedge-shaped detector modules. They provide full azimuthal coverage in the complete rapidity range. They also provide some radial information coming from stereo modules in the rings 1,2 and 5. These modules consist of two planes of silicon sensors of which one is tilted by $100 \mathrm{mrad}\left(5.7^{\circ}\right)$. The barrel part is subdivided into an inner (TIB) and an outer (TOB) part. The inner part has 4 layers with full azimuthal coverage and stereo modules in layers 1 and 2. The outer barrel has 6 layers, the 2 innermost being equipped with stereo modules. To optimize the transition between barrel and endcaps, two inner endcaps (TID) are foreseen, each one consisting of 3 disks equipped with 3 rings of sensors.

The tracker performance depends on the number of points per track that can be measured. Figure 1.6a shows the number of points collected in the silicon microstrip tracker as a function of the rapidity. The tracker will be able to reconstruct isolated high $p_{t}$ tracks with an efficiency better than $95 \%$ and high $p_{t}$ tracks within jets with an efficiency better than $90 \%$. The occupancy in the tracker has to be $1 \%$ or less to allow for efficient track finding. This limits the strip length of the innermost silicon modules. The momentum resolution for high $p_{t}$ leptons is $\Delta p_{t} / p_{t}=0.1 p_{t}$, where $p_{t}$ is given in $\mathrm{TeV}$. For the impact parameter a resolution of $35 \mu \mathrm{m}$ is achieved in the plane perpendicular to the beamline. Along the beams this resolution goes up to $75 \mu \mathrm{m}$.

The tracker has been designed to be as light as possible. This is crucial to keep multiple scattering of the charged particles as small as possible and to minimize the material inserted in front of the calorimeters. Figure $1.6 \mathrm{~b}$ shows the tracker material in radiation lengths as function of the rapidity [15]. At $\eta \approx 1.2$ it peaks due to the routing of cables and cooling pipes inside the tracker volume.

\footnotetext{
${ }^{1}$ In its first design, this part of the tracker contained two types of sensors, silicon microstrip detectors and microstrip gas chambers (MSGCs), as described in the technical design report (TDR) [13]. The advantages of having a single sensor technoogy for the whole microstrip tracker led to the decision to replace the MSGCs with silicon microstrip detectors. This change has been described in an addendum to the TDR [14].
} 


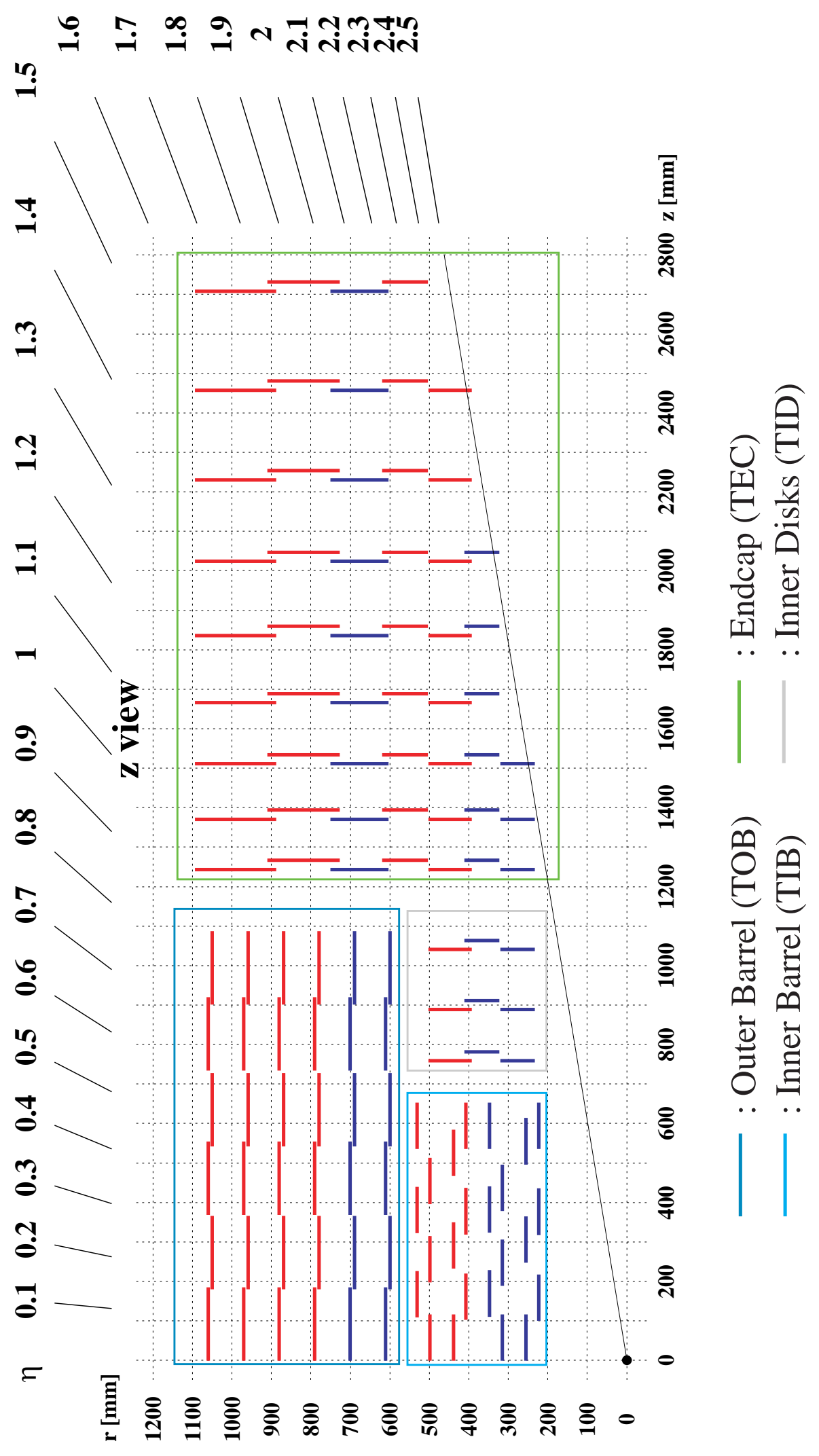

Figure 1.5: General layout of the CMS silicon microstrip tracker. The single-sided modules are marked in red, the stereo modules in blue. 

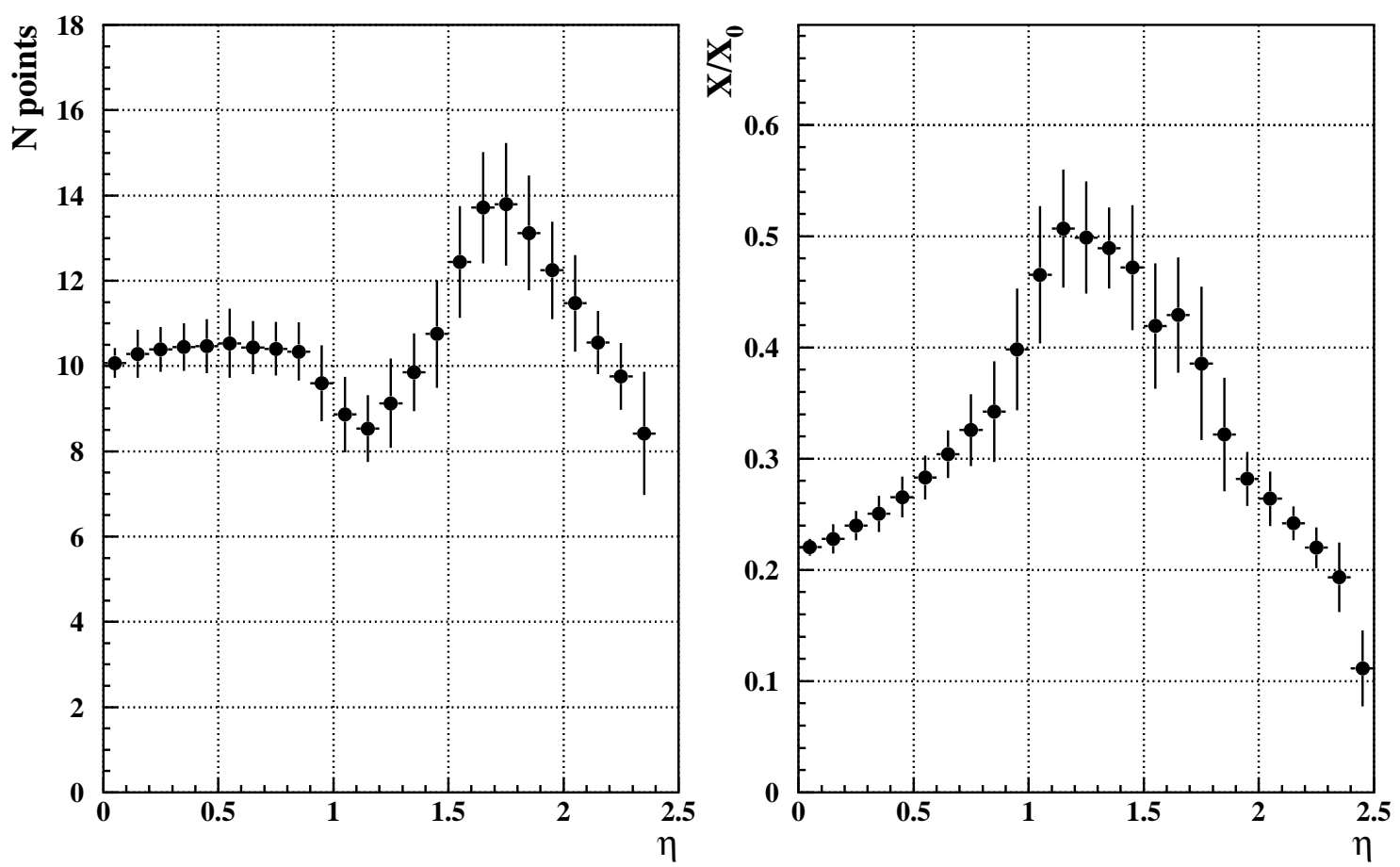

Figure 1.6: a: Number of points collected by tracks as a function of rapidity. Pixels are not included and stereo points are counted once. b: Material budget excluding pixels and material outside the tracking volume. [15] 


\subsubsection{The silicon strip detectors}

The working principle of a silicon strip sensor is sketched in fig.1.7a . It consists of a p-n junction to which a reverse bias voltage is applied. This removes the charge carriers out of the silicon and generates an electric field in the bulk. The voltage at which the electric field reaches through the entire sensor is called depletion voltage. A charged particle crossing the sensor will generate a track of electron-hole pairs in the depleted region. These charge carriers then drift in the electric field, inducing a current pulse in the electrodes. The pulse is fed through a coupling capacitor into a charge sensitive amplifier after which it is further processed by the readout system. The p-n junctions are structured into strips, each connected to its own preamplifier, thus allowing a position-dependent measurement.
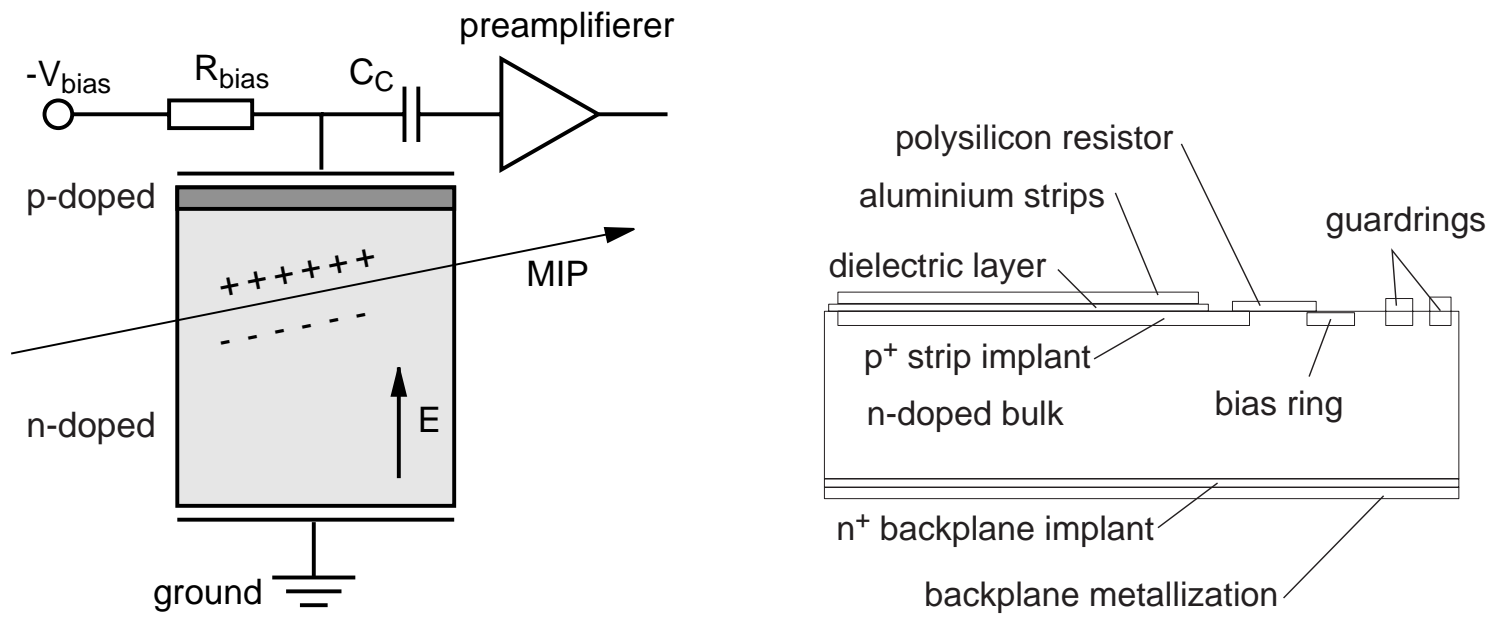

Figure 1.7: a) Working principle of a silicon particle detector. b) Schematic structure of the CMS silicon strip sensors

\section{The Sensors}

The silicon sensors that will be used in the CMS tracker [16] are based on n-type silicon with a resistivity of $3-5 \mathrm{k} \Omega$. The strips are $\mathrm{p}^{+}$implants which are processed on one side of the sensor (see fig.1.7b). The other side (backside) is coated with an unstructured $\mathrm{n}^{+}$implant and covered with an aluminium layer for protection and electrical contacting. The strips are connected to a bias ring via integrated polysilicon resistors. Strips and bias ring are surrounded by a set of guard rings which improve the breakdown performance of the sensors. The strip implants are covered with a dielectric (thin layers of $\mathrm{Si}_{3} \mathrm{~N}_{4}$ and $\mathrm{SiO}_{2}$ ) to achieve $\mathrm{AC}$ coupling. On top of the dielectric layer come the metal strips connected to the frontend chips. The capacity between metal and $\mathrm{p}^{+}$strips is $>1.2 \mathrm{pF}$ per cm strip length and $\mu \mathrm{m}$ strip width, giving rise to total coupling capacities from $200 \mathrm{pF}$ to $1 \mathrm{nF}$. 


\section{Radiation Hardness}

Due to the high luminosity of LHC, one major concern for the operation of the detector will be radiation damage. The silicon sensors are required to operate at up to $500 \mathrm{~V}$ before and after irradiation. The integrated flux throughout the 10 years of LHC operation will be $2.4 \cdot 10^{14} \mathrm{~cm}^{-2}$ of $1 \mathrm{MeV}$ equivalent neutrons for the innermost strip sensors. There are three important parameters which will change as a function of the irradiation:

- Leakage current

- Depletion voltage $V_{d e p}$

- Charge collection efficiency

The leakage current increases by several orders of magnitude during the 10 years of operation. The dissipated heat has to be cooled away effectively to avoid thermal runaway of the sensors.

The depletion voltage changes due to the effective doping concentration $N_{\text {eff }}$ in the bulk silicon. The non-ionizing energy loss of the particles crossing the sensors will cause lattice damage in the silicon, which results in an effective introduction of acceptors in the material. Therefore the originally n-type doping will slowly decrease, become zero, and with further irradiation change to p-type doping. This phenomenon is called type inversion [17]. The $p-n$ junction before type inversion will be between the $\mathrm{p}^{+}$strips and the n-type bulk, while after irradiation it will be formed by the $\mathrm{n}^{+}$backplane and the $\mathrm{p}$-type bulk. Since the depletion voltage $V_{d e p}$ is proportional to $N_{e f f}$, it will decrease before type inversion and then rise continuously. The starting doping concentration of the sensors is chosen such that depletion voltage will always stay below $500 \mathrm{~V}$ during the 10 years of LHC operation.

The introduction of effective acceptors into the silicon bulk will continue, even if irradiation has stopped [18]. This phenomenon is called reverse annealing, and it will accelerate the changes of $V_{d e p}$. Since this effect is strongly dependent on temperature, it can be effectively controlled by keeping the silicon at cold temperatures. For this reason, and to avoid the thermal runaway, the silicon modules are permanently kept at $-10{ }^{\circ} \mathrm{C}$, except for short periods of maintenance.

The charge collection efficiency also degrades during irradiation, due to the introduction of lattice defects which may trap the charge carriers. This loss of charge can be partially recovered by operating the sensors above their depletion voltage.

\section{The Tracker Modules}

The silicon sensors are assembled onto a carbon fiber frame, which also carries the front-end electronics (fig.1.9). The stereo modules are made out of two stacked single-sided modules. The strips of the two modules form an angle of $100 \mathrm{mrad}$ to each other. A module from the inner barrel and one from the endcaps is shown in fig.1.8. The carbon fiber used for the frame has a very high thermal conductivity 
$\left(\approx 400 \mathrm{~W} \mathrm{~m}^{-1} \mathrm{~K}^{-1}\right)$ to improve the cooling performance. To keep the warmest spot on the silicon wafers at $-10{ }^{\circ} \mathrm{C}$ the cooling system circulates its fluid at $-20{ }^{\circ} \mathrm{C}$. The modules have precision inserts, which fit into matching pieces on the mechanical structure. The strips are up to $200 \mathrm{~mm}$ long, the strip pitch being $80 \mu \mathrm{m}$ for the innermost modules and $200 \mu \mathrm{m}$ for the outermost.
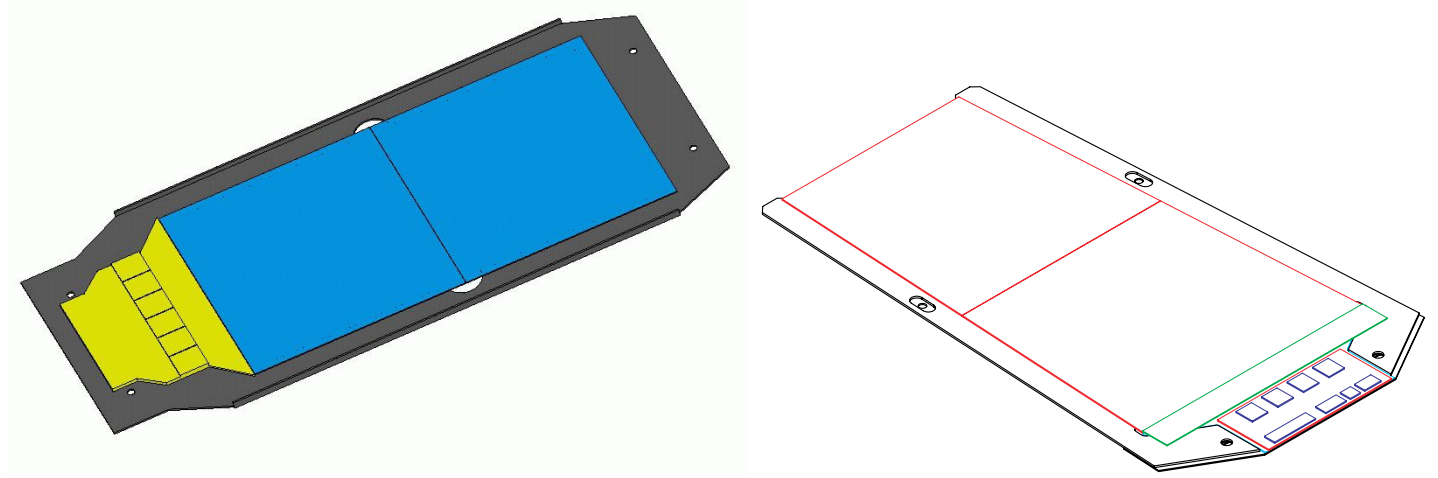

Figure 1.8: Silicon microstrip modules for the inner barrel and the endcap.

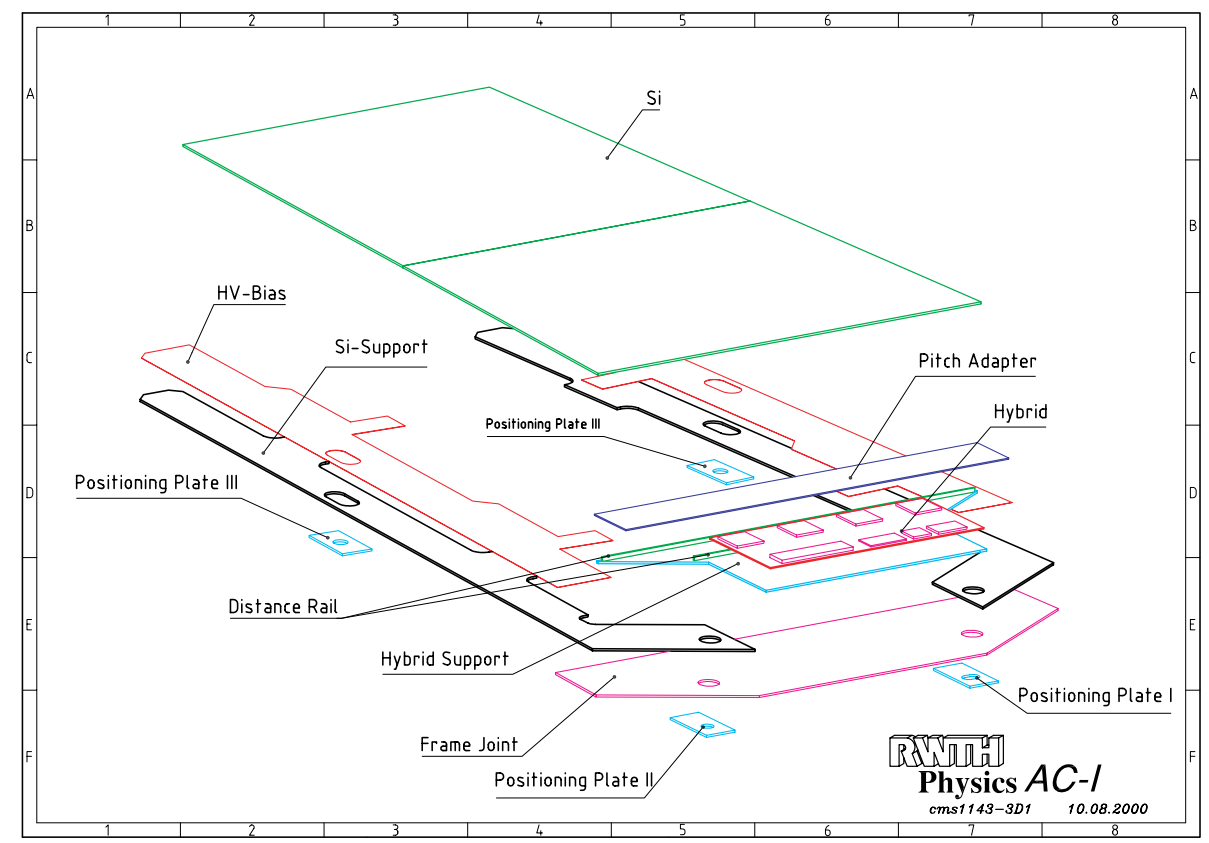

Figure 1.9: Exploded 3D-View of an endcap tracker module. 


\subsubsection{The Mechanical Support Structure}

The CMS silicon microstrip tracker is organized into 4 subdetectors:

- Outer barrel

- Inner barrel

- Two endcaps

- Two mini-endcaps

The outer barrel consists of 4 flanges (fig.1.10). So-called rods stretch out in between these flanges, connecting them. The rods support the detector modules and route the services to them. The inner barrel is built up in a similar way, having

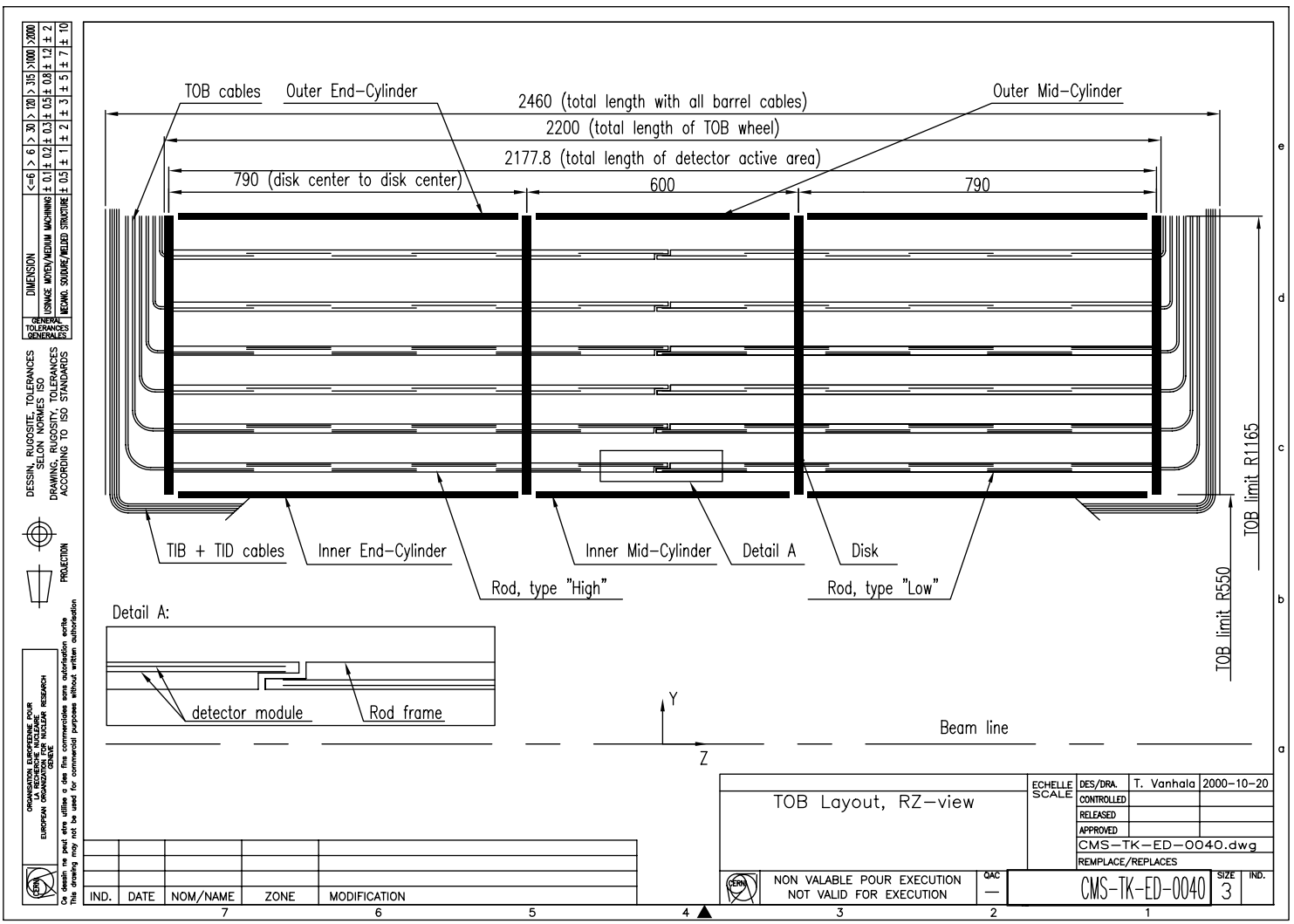

Figure 1.10: Mechanical support structure of the Tracker Outer barrel (TOB).

cylindrical carbon fiber sheets instead of the rods. These sheets support the modules and have many openings to save weight and run cables and cooling pipes through them (fig.1.11 left). The endcaps have 9 disks made out of honeycomb sandwiched in between two carbon fiber skins (fig.1.11 right). Each disk carries 16 so-called petals, which are wedge-shaped structures made out of thinner honeycomb plates. These petals support the modules and the services (fig.1.12). The cooling pipes 
are integrated into the honeycomb structure and are in thermal contact with the modules. Specially designed printed circuit boards route the power and signal lines from the outer rim of the petals to the modules. These boards also carry optical drivers and control electronics.

The detector modules are fixed on the mechanical support structure with precisely machined inserts. In this way, the precision of the assembly of the modules onto the mechanical structure can be brought down to $\approx 50 \mu \mathrm{m}$. Following the assembly it is planned to perform a survey in which the position of the modules is measured with a coordinate measuring machine. After this survey the relative position of the modules with respect to each other will be known with a precision of $\approx 20 \mu \mathrm{m}$.
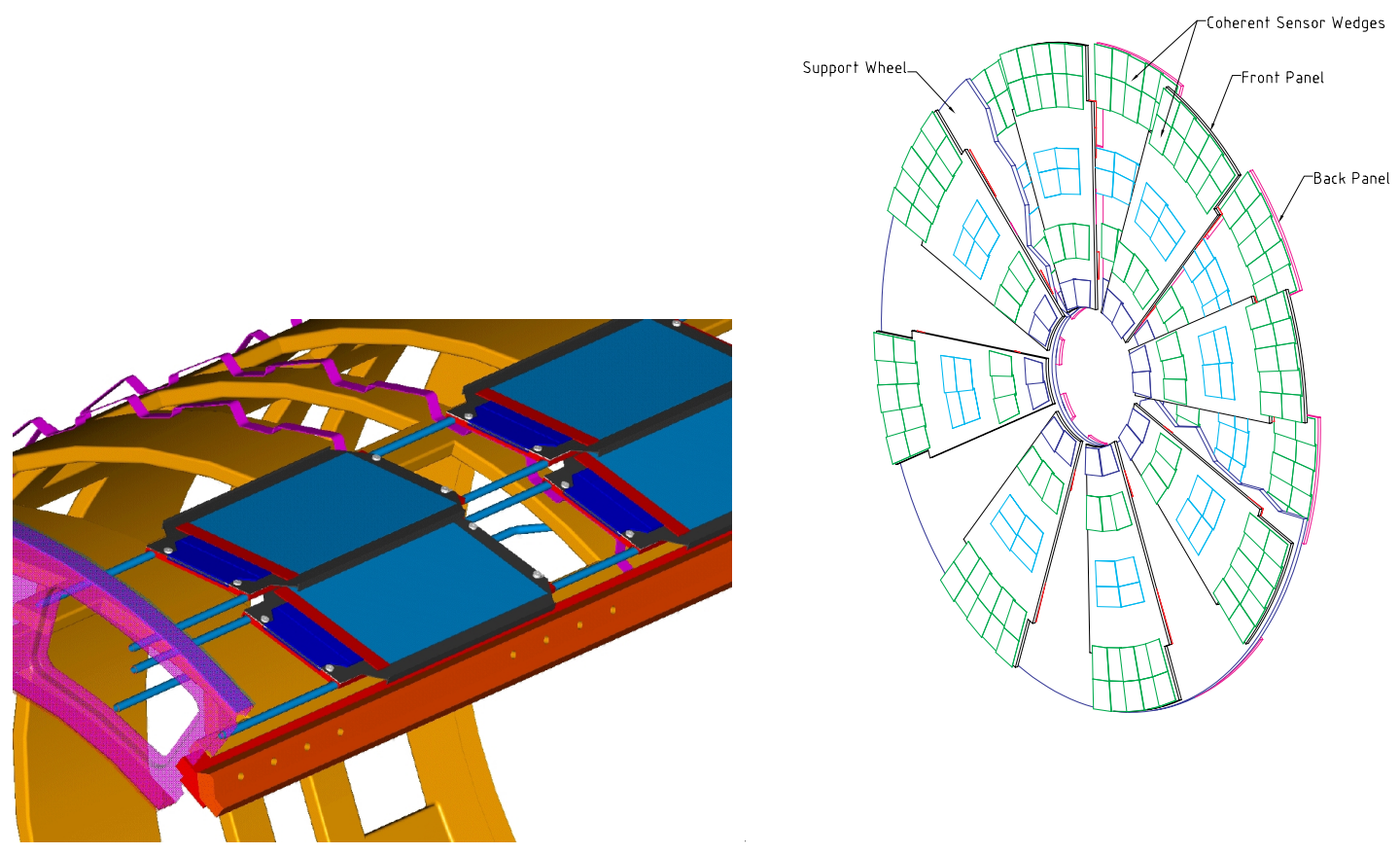

Figure 1.11: Left: 3D detail of the mechanical support structure of the inner barrel (TIB). The modules are supported on carbon fiber half cylinders which have openings to save weight and route the services to the modules. Right: Endcap disk with the petal layout. The wedge-shaped sensors are mounted on the petals. 


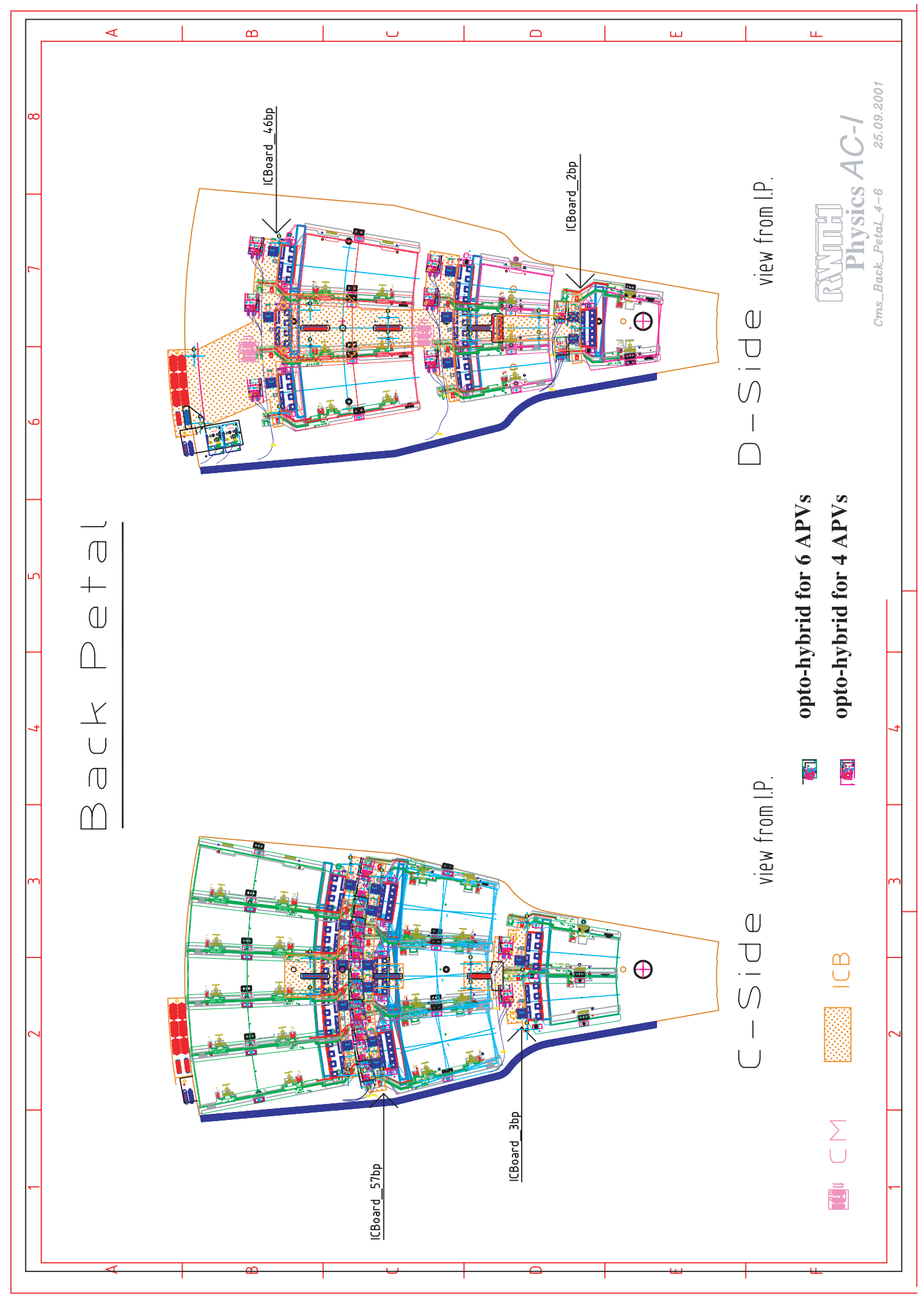

Figure 1.12: Endcap petal supporting the modules. 


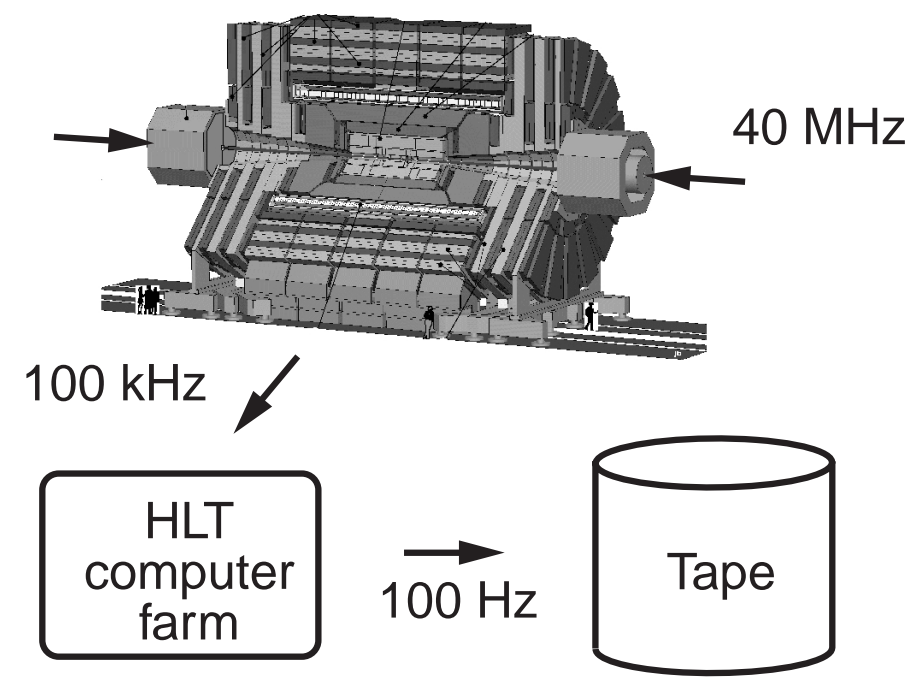

Figure 1.13: The CMS trigger is organized into several levels. Level 1 is a hardware and the higher level triggers (HLT) are software triggers. For the HLT data of reconstructed tracks is used. This makes it necessary to do part of the track reconstruction online.

\subsection{Purpose of the Alignment System}

There are two main reasons, why a good inter-alignment of the CMS tracker is required, namely the need for online track reconstruction and the precise measurement of track momenta.

The first reason is a most crucial one and has to do with the CMS triggering scheme (see fig.1.13). The CMS trigger is organized into several levels [19]. The first level trigger is implemented in the hardware of the detector. It evaluates informations coming from the muon system and the calorimeters, allowing to trigger on high $p_{t}$ muons, jets, photons and missing energy. This trigger will reduce the $40 \mathrm{MHz}$ bunch crossing rate to a maximum of $100 \mathrm{kHz}$ of accepted triggers. At this rate the CMS detector is read out, and the data is fed into a computer farm. Here the high level triggers (HLT) are applied, bringing the event rate which is written to tape down to $100 \mathrm{~Hz}$. These triggers, which are software implemented, make use of the tracker data. This makes it necessary to reconstruct part of the tracks online at rates up to $10 \mathrm{kHz}$. Powerful pattern recognition algorithms have been developed for this purpose. These algorithms can only work properly, if the position of the sensors is known to a precision better than a few $100 \mu \mathrm{m}$ [20].

Also the precise measurement of the track momenta requires a good alignment of the silicon modules. Since this evaluation is done offline, one could in principle use track data to align the sensors. To do this effectively however, a good starting 
position for the modules is needed that could be supplied by an alignment system.

There are several sources of possible deformations of the tracker mechanical structure. The tracker is assembled at room temperature and operated at $-10{ }^{\circ} \mathrm{C}$, the cooling pipes being even colder $\left(-20^{\circ} \mathrm{C}\right)$. This will cause thermal stresses due to different thermal expansion coefficients. Gravitational forces may cause sagging of parts of the tracker structure which is supported only at a few points. Stresses also arise from the many cables and pipes which bring the services into the tracker and carry the data signals to the data acquisition. Finally moisture adsorption may also deform the carbon fiber parts.

In principle the mechanical structure of the tracker is designed in a way to keep deformations below the critical few $100 \mu \mathrm{m}$. But there are several unknowns, which make it advisable to monitor possible deformations. First of all, the large size of the CMS silicon tracker is unique, with its overall length of $6 \mathrm{~m}$ and a diameter of $2.5 \mathrm{~m}$. There is little experience on the behavior of the carbon fiber of such large and light structures over the planned operation period of 10 years. Furthermore, the high irradiation doses may change the material properties in an unforeseen way. If for some reason the cooling power has to be increased, the thermal stresses will become larger.

Since the good alignment of the modules is crucial for the CMS trigger, it is preferrable not to rely only on the stability of the design, but to introduce some additional alignment system. This system should fulfill several requirements. First of all it should measure possible deformations with an accuracy of $100 \mu \mathrm{m}$ or better. Then there should be a straightforward way to relate the measured deformations to the track data. The introduction of additional components should be kept to a minimum to save on the material budget. The system should be simple enough to be easily integrated into the existing data acquisition system. Finally this system should remain financially affordable. In the next section the working principle of such an alignment system based on infrared laser beams is presented.

\subsection{Working Principle of the Alignment System}

The alignment system foreseen for the CMS tracker was inspired by a similar system implemented in the AMS experiment[21, 22]. Its concept is based on the fact, that silicon is partially transparent to infrared light. If the wavelength of the light is chosen carefully, the absorbed light will generate a signal in the silicon sensor, while the transmitted light can travel on to another sensor. In this way one can traverse several layers of Si-microstrip detectors with laser beams (see fig.1.14). These beams are straight tracks and every deviation from a straight line measured in one of the layers, indicates a displacement or deformation of the mechanical support structure. Since the laser beam hits sensors on several layers, it becomes possible to separate the displacement of one layer from movements of the beam. With several laser beams passing through the tracker, it becomes possible to measure the global deformations of the mechanical support structure. 


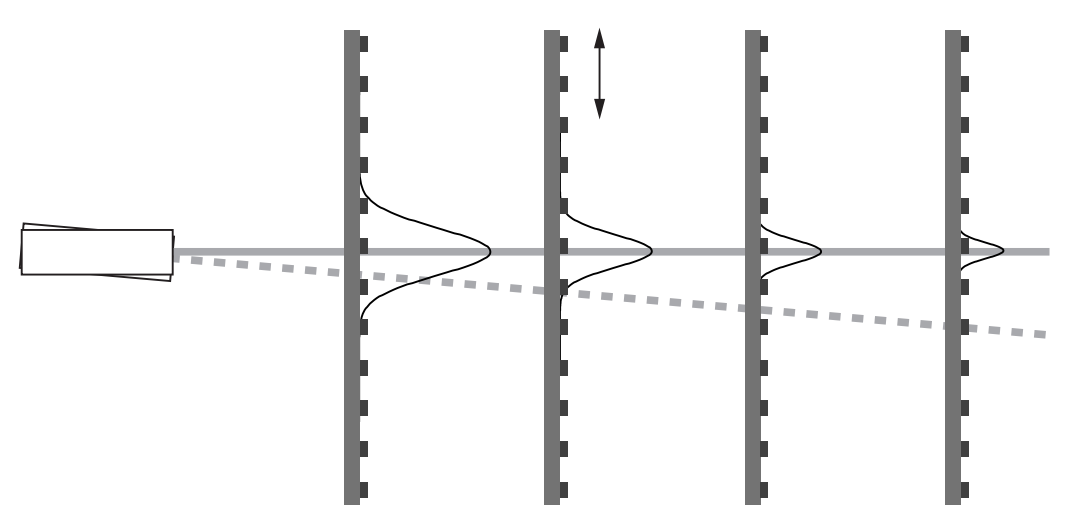

Figure 1.14: Sketch of the working principle of the alignment system. The laser beam passes through several silicon layers, allowing to measure the displacement of each layer. Beam tilts can be separated from individual layer movements.

One key feature of such a system is that the same modules which measure the particle tracks are also used as alignment sensors. In this way the additional material is kept to a minimum, reducing costs and keeping the material budget small. It also simplifies the task of transferring the measured deformations to correction parameters for the particle tracks.

In the next chapter the design considerations for the alignment system will be discussed in detail. Section 2.1 will treat the requirements imposed on the light sources, followed by a section dedicated to the preparation of the silicon sensors used for the alignment. In section 2.3 the absorption of light in silicon will be discussed, together with the reflection and interference effects occuring when light passes through a sensor. This will allow the calculation of the overall amount of light transmitted through one sensor. The subsequent section deals with refraction of the laser beams and the consequences for the alignment system. The chapter ends with section 2.5 consisting of a discussion of the accuracy with which one can find the laser spot on a sensor and the precision that can be achieved in reconstructing beam and sensor movements.

Chapter 3 will then present the laser alignment system for the CMS tracker. In a first section the general layout is described. The next section will apply the results of chapter 2 to the layout and discuss the consequences for the alignment system. Section 3.3 will then treat in detail the reconstruction of the tracker deformation from the measured data. In 3.4 a simulation of the performance of the alignment system will be described, followed by a section presenting and discussing the simulation results.

Finally chapter 4 will give a summary of this work and point out some open issues on which further work has to be done. 


\section{Chapter 2}

\section{Design Considerations for the Alignment System}

In order to design an alignment system which meets the required precision, we have to discuss in detail the aspects which affect the performance of the system. First of all a suitable light source has to be identified and the sensors have to be made transparent. Then there are several effects which take place when a laser beam passes through a silicon layer:

- Reflection and absorption limit the amount of light that is transmitted through one sensor.

- The amount of signal induced in one sensor is proportional to the absorption.

- The interference of the reflections at the two silicon-air interfaces changes the absorbed and transmitted intensities.

- Refraction at the silicon-air interfaces may create a kink in the originally straight laser beam.

For the alignment system the ultimatively relevant figures are the accuracy with which the position of the laser spot is measured on the module, and the kinks which are induced in the beams when passing through a silicon layer. These kinks, which are caused by refraction, will be discussed in section 2.4. The position accuracy is a function of the signal-to-noise $(\mathrm{S} / \mathrm{N})$-ratio, the beam size and is also affected by deformations of the beam profile. By adjusting the beam intensity, the beam diameter and by averaging over many laser pulses, the influence of the S/N-ratio can be made negligible. The remaining deformations of the beam profile, caused by interference, will then be the dominant effect.

\subsection{Light Sources}

There are several requirements that the light source of the alignment system should fulfil. The emitted wavelength should be in the infrared range from 1000 to $1100 \mathrm{~nm}$. 
For these wavelengths the light absorption in silicon is small enough for a beam to cross several layers, while still enough energy is deposited to induce a useful signal. The light should be pulsed and the pulselength be shorter than $40 \mathrm{~ns}$, to be compatible with the readout electronics of the tracker modules. To get enough signal in this short time, the energy density should be high. Last but not least, we need a stable beam with a gaussian profile, to get a good precision in finding the beam spot on the microstrip sensors. All these requirements are met by lasers, of which the most promising candidates are laser diodes and Nd-YAG lasers.

\section{Laser diodes}

With laser diodes it is easy to achieve pulse lengths shorter than 40 ns. With typical optical emission powers of a few $10 \mathrm{~mW}$, one obtains pulse energies in the order of $1 \mathrm{~nJ}$. An advantage of laser diodes is the possibilty of triggering with very small time jitter $(<1 \mathrm{~ns})$. This makes it easy to integrate and synchronise them in the electronic readout system. Laser diodes in the required spectral range have the big disadvantage of being rare. The infrared diodes used in telecommunications have typically wavelengths of $980 \mathrm{~nm}, 1300 \mathrm{~nm}$ and $1550 \mathrm{~nm}$. There exist diodes emitting at $1060 \mathrm{~nm}$ and $1080 \mathrm{~nm}$, which are used to simulate Nd-YAG lasers. Due to their rather exotic status, they are quite expensive. Nevertheless they are possible candidates for the alignment system.

\section{Nd-YAG laser}

The Nd-YAG laser is a solid state laser based on a $\mathrm{Nd}^{3+}$-doped Yttrium-Aluminium garnet. It is a 4-level laser with an emission wavelength of $1064 \mathrm{~nm}$. This wavelength lies within the range of suitable photon energies for the alignment system. For this application we chose a pulsed laser with passive Q-switched crystal. Typical pulse lengths are shorter than $20 \mathrm{~ns}$ at a repetition rate up to $5 \mathrm{kHz}$. The pulse energy lies around a few $\mu \mathrm{J}$. This high pulse energy makes it possible to get all the needed beams for the alignment system out of one single laser, reducing the costs dramatically. One disadvantage of the Nd-YAG laser however, is the large time jitter in triggered mode. This comes from the time variations in the pumping process. Using diode pumped lasers reduces the jitter, but it still lies around $200 \mathrm{~ns}$, being too large for the CMS readout system, which is clocked with $40 \mathrm{MHz}$. It is therefore necessary to derive a triggering signal from the emitted light pulses. With fast photodiodes this can be done easily with a time jitter $<2$ ns, at the expense of losing synchronisation with the readout system. The consequences for the alignment system arising from this are dsiscussed in section 3.1.

\section{Laser stability}

Most of the measurements presented in this work were performed with a Nd-YAG laser. We therefore take a closer look at the stability of this laser. For the laser 
alignment system, it is important to have a source with a stable beam profile. Furthermore, it is helpful if the beam profile is gaussian, since it is easy to fit a curve to such a profile. This gives better precision in finding the position of the laser spot than a simple center of gravity algorithm. To achieve a clean gaussian profile one needs a beam with just one mode. We therefore consider to transport the light with single mode fibers.

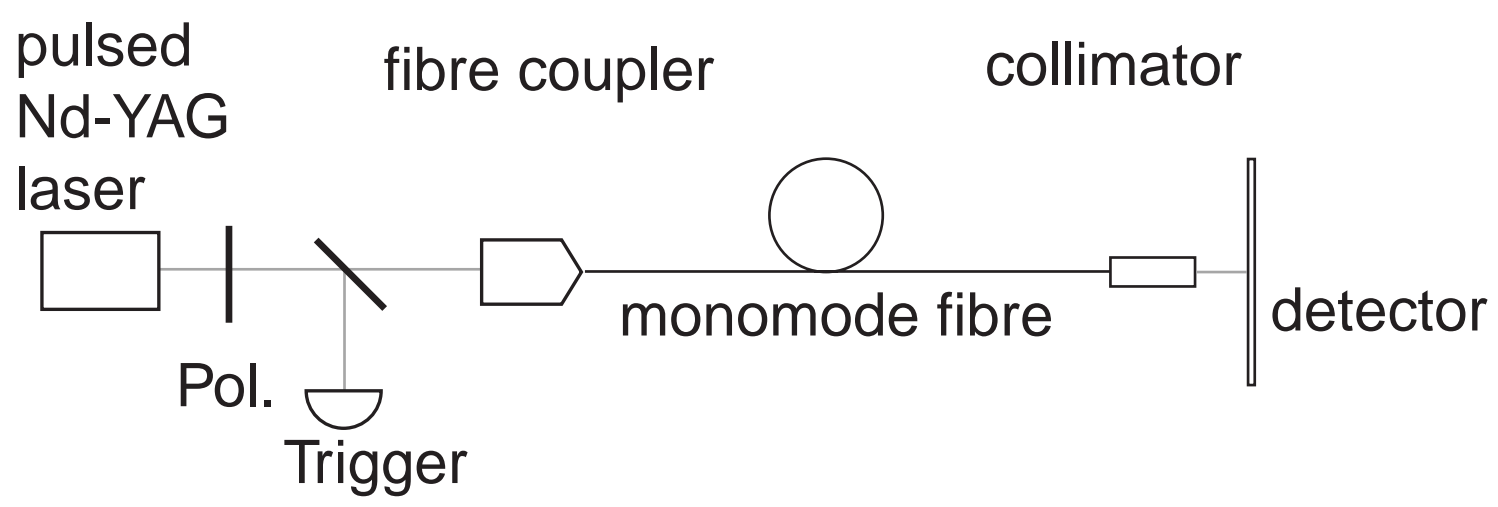

Figure 2.1: Setup for measuring the laser stability

The test setup to measure the profile stability is sketched in fig.2.1. The laser beam is aimed onto a microstrip detector, which is read out by a VME data acquisition system. The triggering signal is obtained by splitting the beam and sending half of it to a fast photodiode. The intensity can be adjusted with the help of a polarizing filter which is brought into the beam. The beam shines always on the same spot, and a few hundred pulses are sampled. The typical pulse shape coming from the front-end electronics is shown in fig.2.2 a). The pulse height on the different strips is shown in fig. $2.2 \mathrm{~b}$, displaying a clean gaussian profile. The data points are the measured ADC-values and the solid line is a gaussian fitted to the data. Note that these measurements were made in a region of the sensor with homogeneous thickness. Variations in the thickness would distort the profile, as will be discussed in section 2.3.4. To quantify the profile stability a gaussian curve was fitted to each sampled pulse. The distributions of the obtained fit parameters are shown in fig.2.3 and 2.4. As can be seen, the width and height of the profile vary only on the percent level, while the position stays the same to the level of $\approx 1 \mu \mathrm{m}$. The Nd-YAG laser is thus well suited for the alignment system. 

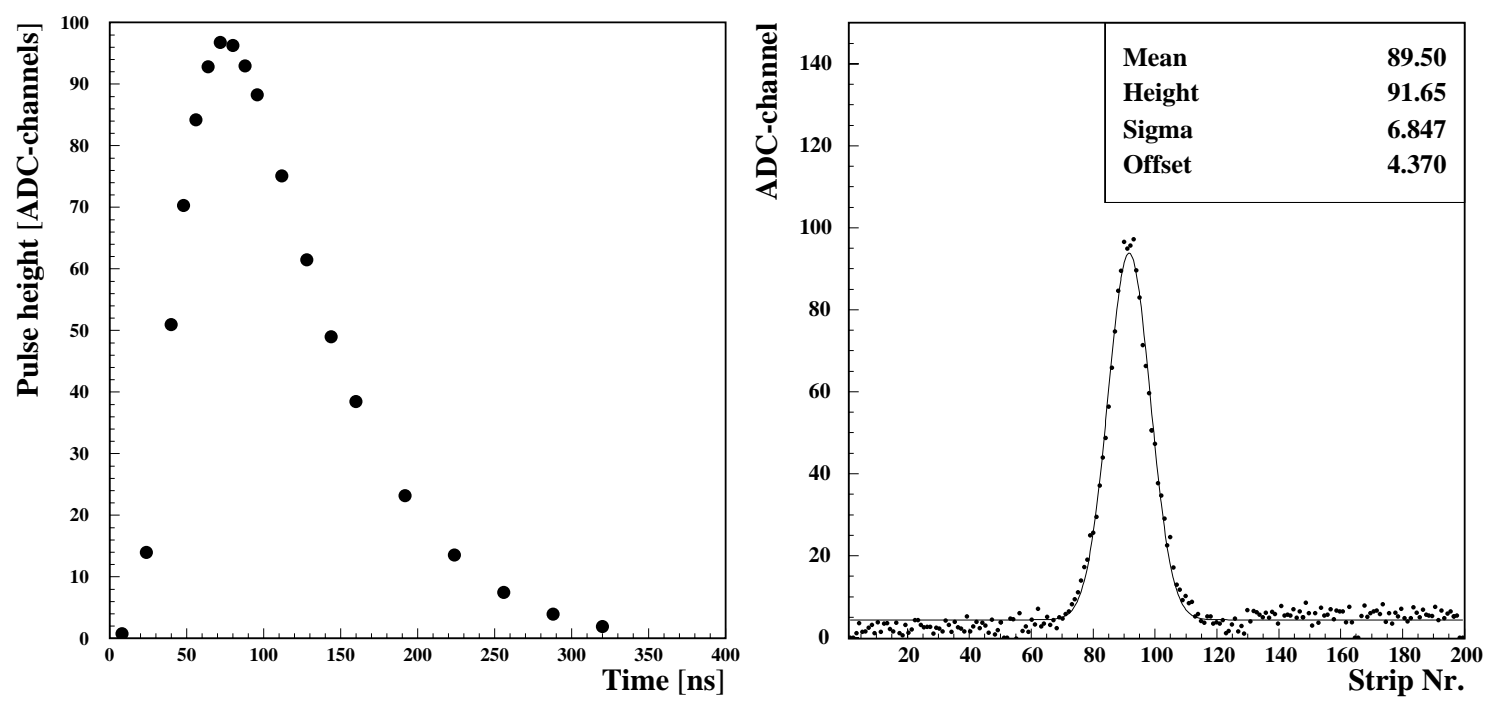

Figure 2.2: a) Electrical pulse at the end of the fromt-end amplifier from a laser pulse signal. b) Laser profile measured by a silicon microstrip detector.
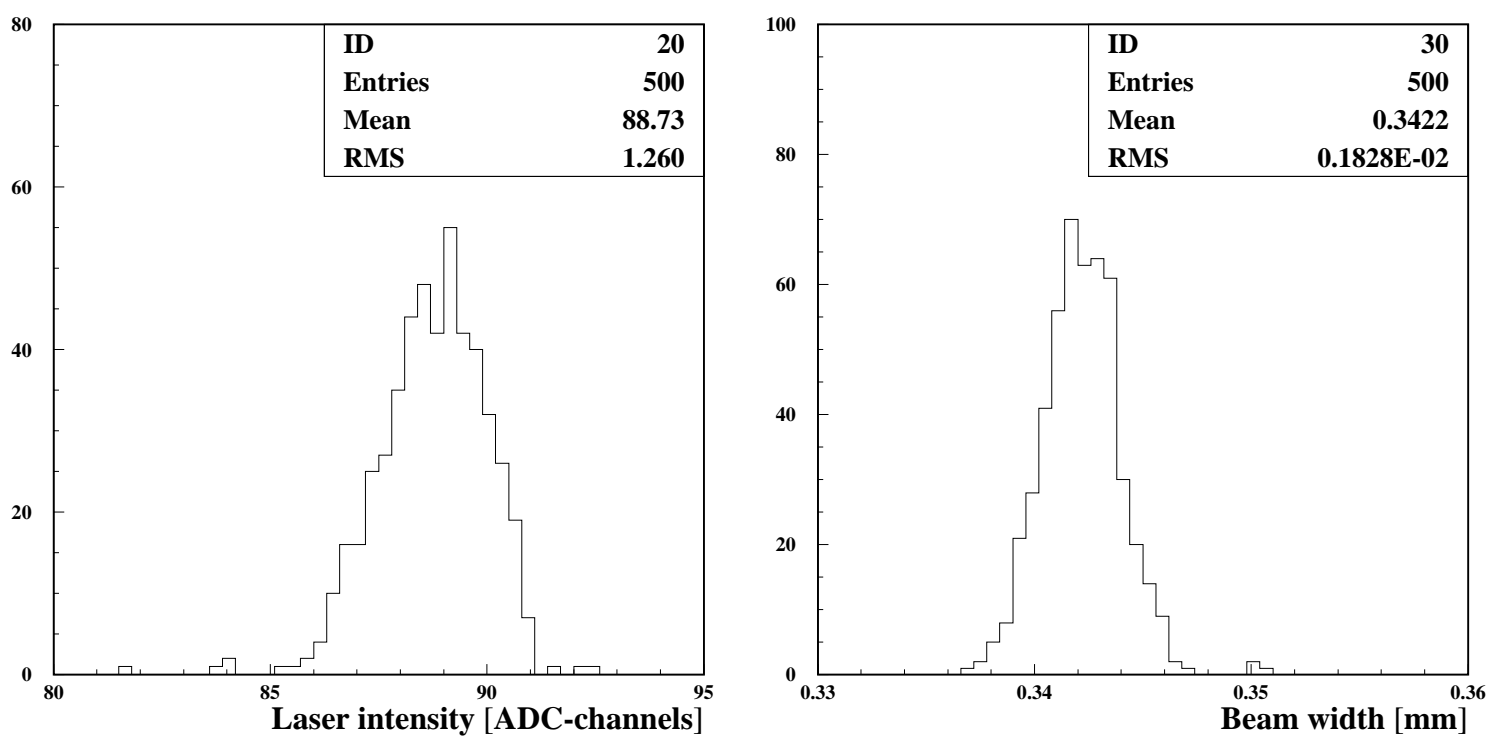

Figure 2.3: The distributions of the height ( $\propto$ laser intensity) and the $\sigma$ of the gaussians fitted to the laser pulses. 


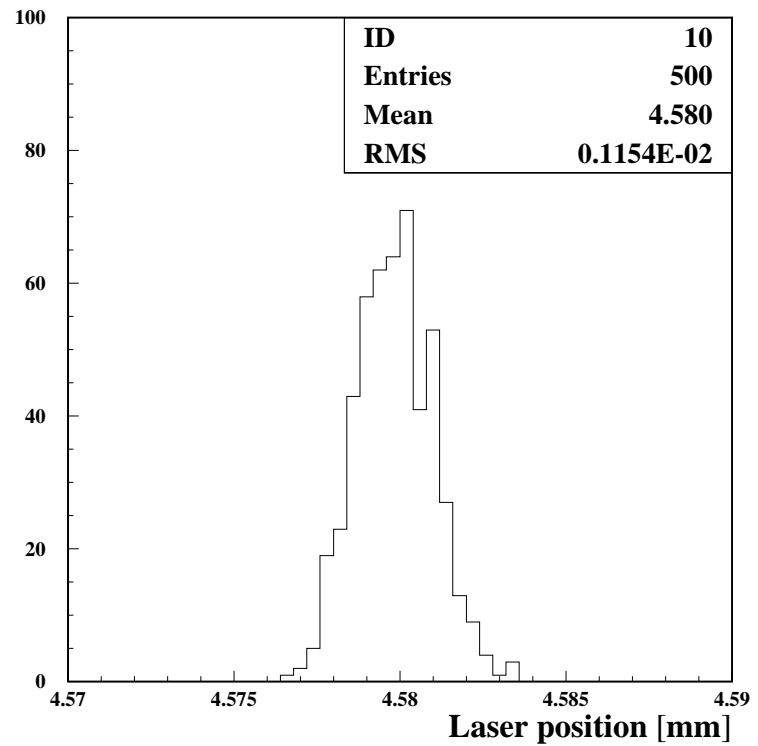

Figure 2.4: The distributions of the fitted laser spot. 


\subsection{Transparent Detectors}

In section 1.4.2 a description of the silicon sensors used in the CMS tracker was given. Fig. 2.5 shows a section of such a sensor perpendicular to the strips. Except for the aluminium on the front- and backside, all materials are transparent for infrared light. To get useful sensors for the alignment system, the metallization

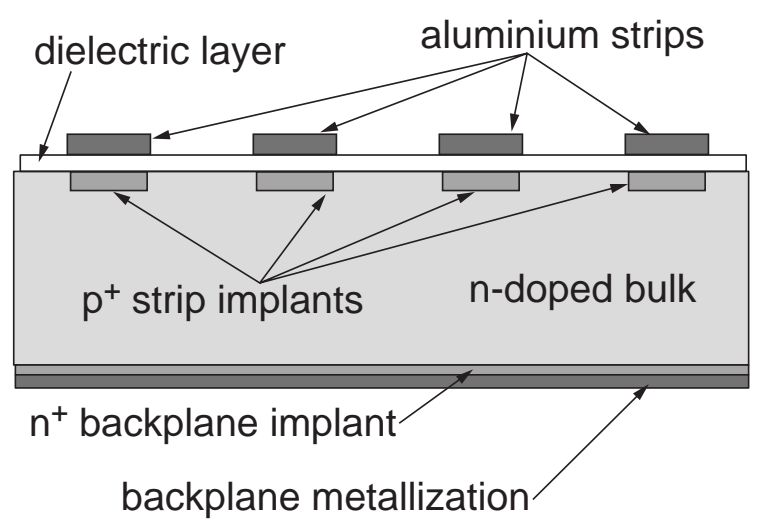

Figure 2.5: Section of a silicon strip sensor. Except for the aluminium, all parts are transparent for infrared light.

on the backplane has to be removed in the region where the laser beam will pass through the sensor. This does not affect the functioning of the sensor, since it is the $\mathrm{n}^{+}$implant which forms the ohmic contact before and the junction after type inversion. The $\mathrm{n}^{+}$implant has a very high charge carrier density and thus a good electrical conductivity, so that the region without metallization remains electrically connected to the rest of the backplane. The aluminium strips on the frontside cannot be removed, even in a small region, because an interruption in the strip metallization would reduce the capacity of the coupling capacitors.

The metallization on the backplane is removed by etching away the aluminium (see fig.2.6). This is done with a mixture of acids that act in a selective way, etching away the aluminium and leaving the silicon intact. It consists mainly of phosphoric acid $\left(\mathrm{H}_{3} \mathrm{PO}_{4}\right)$ to which nitric acid $\left(\mathrm{HNO}_{3}\right)$ and acetic acid $\left(\mathrm{CH}_{3} \mathrm{COOH}\right)$ is added. The additives act as buffers to keep the $\mathrm{pH}$-value constant. An alternative way to etching would be to process transparent contacts. This would require special masks and additional steps in sensor processing.

As a further improvement we consider to cover the etched holes with an antireflective coating. This minimizes reflective losses and reduces interference effects (see sect. 2.3). 


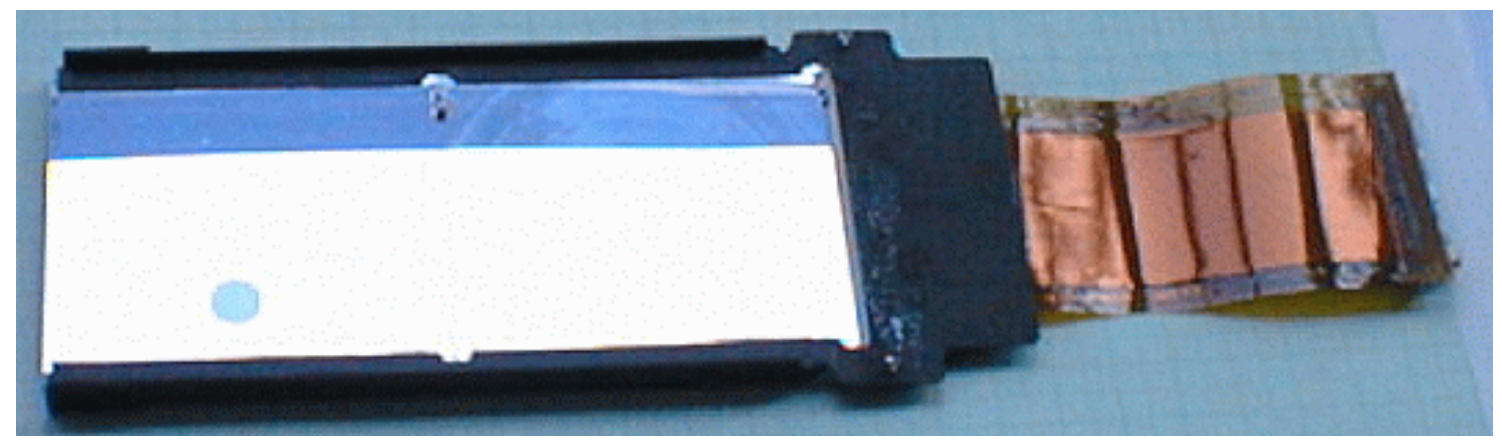

Figure 2.6: Silicon strip sensor with a hole etched into the backplane metallization

\subsection{Transmission and Absorption of the laser beam}

\subsubsection{Absorption in silicon}

The absorption of electromagnetic radiation in a medium is described by the absorption coefficient $K$ :

$$
I(d)=I(0) e^{-K \cdot d}
$$

where $I(d)$ is the intensity at depth $d$. The absorption coefficient is related to the extinction coefficient $k$ by:

$$
k=\frac{K \cdot \lambda}{4 \pi}
$$

$k$ describes the exponential fading of the wave amplitude:

$$
\left|E_{t}(z)\right|=\left|E_{t}(0)\right| e^{-k / z}
$$

In a semiconductor photon absorption takes place through the transition of electrons from the valence band into the conduction band. Silicon is a semiconductor with an indirect bandgap, which means that in addition to the energy that is acquired by the electron from the photon, also a momentumn transfer from a phonon has to take place, for the electron to reach the conduction band. The gap energy $E_{\text {gap }}$ of silicon is temperature dependent, dropping from $1.16 \mathrm{eV}$ at $0 \mathrm{~K}$ to $1.11 \mathrm{eV}$ at $300 \mathrm{~K}$. In the temperature range from $-20{ }^{\circ} \mathrm{C}$ to $+20{ }^{\circ} \mathrm{C}$ the gap energy changes by roughly $1 \%$. Due to the indirect bandgap the absorption of photons in silicon involves phonons and becomes dependent on the phonon spectra. Jellison et al. have derived a formula describing photon absorption in silicon [23]. They consider the temperature dependent gap energy and 3 different phonon energies. The result is shown in fig.2.7, where the absorption coefficient $K$ is plotted as a function of the wavelength. Curves are shown for $260 \mathrm{~K}$ and $300 \mathrm{~K}$. The increased transparency with dropping temperature is due to the increasing energy gap and the reduction of the amount of phonons, the latter being the dominant factor. 


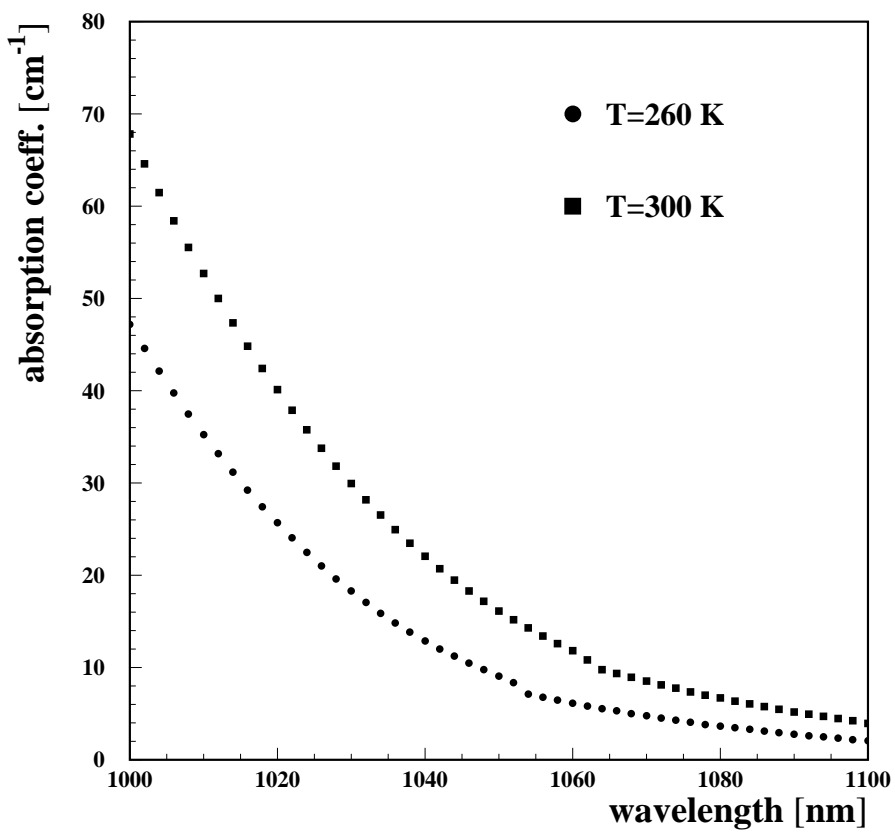

Figure 2.7: Absorption coefficient in silicon after [23].

\subsubsection{Reflection at silicon-air interface}

An electromagnetic wave incident on a boundary of two media with different optical properties is split into a reflected and a transmitted part. The amount of light that is reflected or transmitted depends on the refractive indices of the materials, the absorption coefficients and the polarization of the incident wave. The ratios of reflected and transmitted energies to the incident energy are given by:

$$
\begin{aligned}
R & =\left|\frac{\left(N_{0}-N_{1}\right)^{2}}{\left(N_{0}+N_{1}\right)^{2}}\right| \\
T & =\left|\frac{4 N_{0} N_{1}}{\left(N_{0}+N_{1}\right)^{2}}\right|
\end{aligned}
$$

Here $N_{0}$ and $N_{1}$ are the complex indices of refraction of the two media, which are related to the optical constants by $N_{j}=n_{j}+i \cdot k_{j}$, with $n_{j}$ designating the standard index of refraction and $k_{j}$ the extinction coefficient. For the air-silicon interface, where $n_{0}=1$ and $k_{0} \approx 0$, equations 2.4 and 2.5 reduce to:

$$
\begin{aligned}
R & =\frac{\left(1-n_{S i}\right)^{2}+k_{S i}^{2}}{\left(1+n_{S i}\right)^{2}+k_{S i}^{2}} \\
T & =\frac{4 \sqrt{n_{S i}^{2}+k_{S i}^{2}}}{\left(1+n_{S i}\right)^{2}+k_{S i}^{2}}
\end{aligned}
$$

The extinction coefficient of silicon is $k_{S i} \approx 10^{-4}$ and can thus be neglected in 2.6 and 2.7. We obtain for the air-silicon interface $R=0.31$ and $T=0.69$. It should 
be emphasized, that this values hold for a single interface. Interference effects that occur when the reflection takes place at several interfaces will be discussed in section 2.3.4.

\subsubsection{Measurement of Transmission}

To estimate the intensity which is transmitted through one silicon layer, we consider a simple sheet of silicon with perfectly reflecting strips on one side. $R_{\text {strip }}=\frac{\text { stripwidth }}{\text { pitch }}$ is the fraction of the intensity which is reflected by the strips, $T_{s a}$ is the transmitted fraction at each silicon-air interface and $T_{S i}$ the light transmitted through $300 \mu \mathrm{m}$ of silicon. The overall transmission $T$ through the sensor is then given by $T=(1-$ $\left.R_{\text {strip }}\right) \cdot T_{s a}^{2} \cdot T_{S i}$. Table 2.1 summarizes all these values for the wavelengths of $1064 \mathrm{~nm}$ and $1080 \mathrm{~nm}$. The transmission was measured with the setup sketched in fig.2.8.

\begin{tabular}{|l|c|c|}
\hline & $\lambda=1060 \mathrm{~nm}$ & $\lambda=1080 \mathrm{~nm}$ \\
\hline$T_{S i}$ & 0.70 & 0.82 \\
\hline$T_{s a}$ & 0.69 & 0.69 \\
\hline$T$ & 0.25 & 0.29 \\
\hline
\end{tabular}

Table 2.1: Transmission of light through a silicon layer of $300 \mu \mathrm{m}$ thickness and $R_{\text {strip }}=0.25$. $R_{\text {strip }}$ has the same value for the modules of the final CMS microstrip sensors.

The Nd-YAG laser, which operates in pulsed mode at a pulse rate of $5 \mathrm{kHz}$, shines directly onto the sensor. The laser diode is operated in $\mathrm{cw}$ mode and first coupled into a fiber. At the end of the fiber a collimator forms a parallel beam which shines onto the sensor. The beam shape is measured with a CCD chip in the beam profiler.

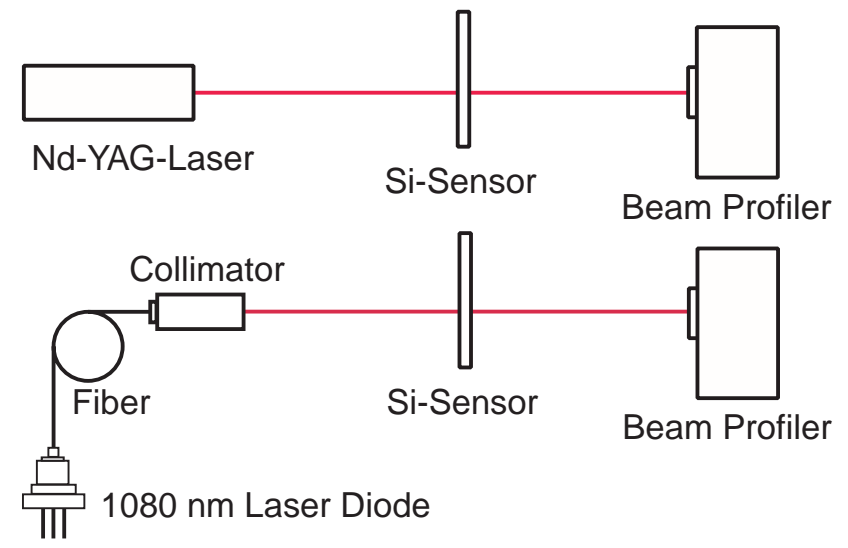

Figure 2.8: Setup for the measurement of the light transmission through a silicon sensor. The Nd-YAG laser operates in pulsed mode, and the $1080 \mathrm{~nm}$ diode in $\mathrm{cw}$ mode. The beam profile is measured with a CCD chip in the beam profiler. 

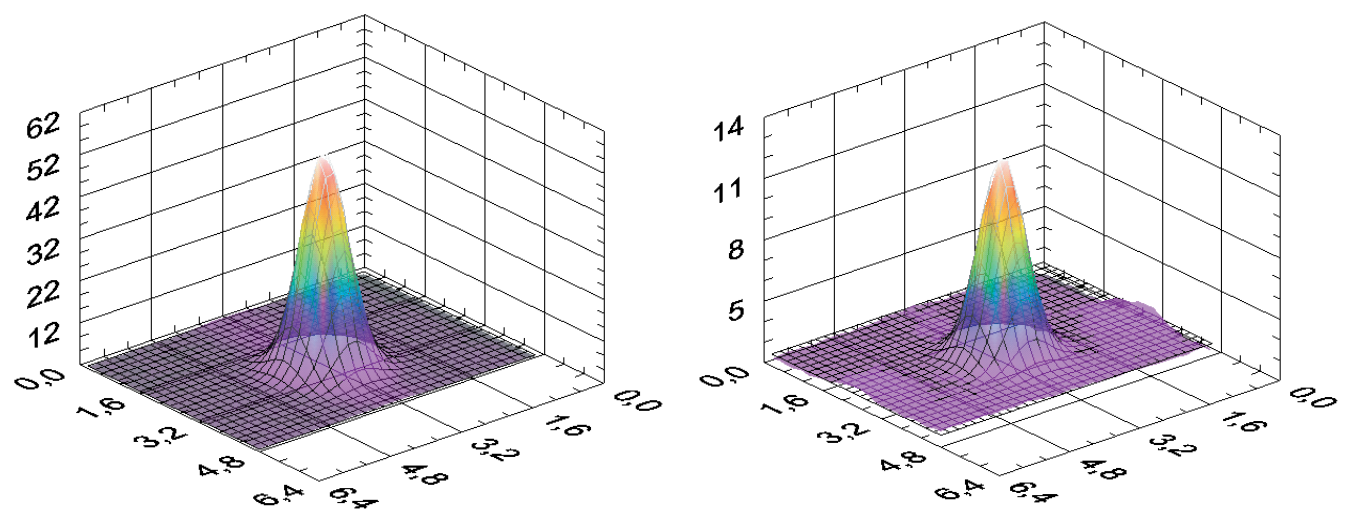

Figure 2.9: Beam profiles measured directly and after passing through a silicon layer. The axes in the horizontal plane give the position in $\mathrm{mm}$, the vertical axis the CCD response in ADC counts. The surface formed by the shades is the measurement, the superimposed grid a two-dimensinal gaussian which was fitted to the data. As transmission coefficient we take the ratio of the volumes under the gaussians, which are proportional to $h \cdot \sigma^{2}$.

A rotation-symmetric gaussian with offset is fitted to the data:

$$
f(x, y)=z_{0}+h \cdot \exp \left(-\frac{\left(x-x_{0}\right)^{2}+\left(y-y_{0}\right)^{2}}{2 \sigma^{2}}\right)
$$

As a measure of the transmitted intensity we take the product $h \cdot \sigma^{2}$ which is proportional to the volume under the gaussian. Fig.2.9 shows two measured profiles, the first being the Nd-YAG laser shining directly onto the beam profiler, while in the second measurement the beam passes through a silicon sensor. The measured values form the shaded surface, while the superimposed grid gives the gaussian fit. Table 2.2 summarizes measurements which were made on different sensors at different locations. The transmission for a wavelength of $1064 \mathrm{~nm}$ is about $20 \%$, and for $1080 \mathrm{~nm}$ we obtain about $24 \%$. The better transmission at $1080 \mathrm{~nm}$ is due to the smaller absorption coefficient at that wavelength. The measured values are a little smaller than the calculated ones from table 2.1 which could be due to the absorption in the dielectric layer between implants and aluminium strips.

\subsubsection{Interference Effects}

If the coherence length of the laser light is longer than $\frac{2 d}{n_{S i}}$, where $d$ denotes the thickness of the silicon layer, then the light reflected on the front- and the backside of the sensor interferes. The multiply reflected laser rays, which exit the sensor on either side, may interfere in a constructive or destructive way (Fig.2.10). As described in sec. 1.4.2, the sensors are covered with a dielectric layer giving rise to additional reflections at the boundary between silicon and the dielectric thus causing further interference. The total intensities, which are reflected, absorbed and 


\begin{tabular}{|c|c|l|l|l|l|}
\hline \multicolumn{6}{|l|}{$1080 \mathrm{~nm}$ Laser Diode } \\
\hline Sensor Nr. & Hole Nr. & $\sigma[\mathrm{mm}]$ & $\mathrm{h}$ & $\mathrm{h} \times \sigma^{2}$ & $\%$ \\
\hline \hline directly & - & 0.472 & 53.05 & 11.8 & 100 \\
\hline 1 & 0 & 0.450 & 12.7 & 2.57 & 22 \\
\hline 1 & 0 & 0.452 & 12.9 & 2.64 & 22 \\
\hline 1 & 0 & 0.482 & 12.9 & 3.01 & 26 \\
\hline 1 & 1 & 0.424 & 15.7 & 2.82 & 24 \\
\hline 1 & 2 & 0.406 & 17.0 & 2.80 & 24 \\
\hline 1 & 3 & 0.420 & 17.0 & 3.00 & 25 \\
\hline 1 & 4 & 0.412 & 15.7 & 2.66 & 23 \\
\hline \hline
\end{tabular}

\begin{tabular}{|c|c|l|l|l|l|}
\hline \multicolumn{2}{|c|}{ Nd-YAG (1064 nm) } \\
\hline Sensor & Hole Nr. & $\sigma$ & $\mathrm{h}$ & $\mathrm{h} \times \sigma^{2}$ & $\%$ \\
\hline \hline directly & - & 0.414 & 58.8 & 10.1 & 100 \\
\hline 1 & 0 & 0.416 & 12.4 & 2.15 & 21 \\
\hline \hline directly & - & 0.414 & 51.7 & 8.86 & 100 \\
\hline 2 & 1 & 0.424 & 9.47 & 1.70 & 19 \\
\hline 2 & 1 & 0.428 & 8.61 & 1.58 & 18 \\
\hline 2 & 1 & 0.410 & 9.79 & 1.65 & 19 \\
\hline 2 & 1 & 0.420 & 8.96 & 1.58 & 18 \\
\hline 2 & 1 & 0.446 & 9.29 & 1.84 & 21 \\
\hline
\end{tabular}

Table 2.2: Transmission through $300 \mu \mathrm{m}$ thick sensors measured for a laser diode with $\lambda=1080 \mathrm{~nm}$ and a NdYAG laser with $\lambda=1064 \mathrm{~nm}$. The transmission was measured at several different locations on the sensors.

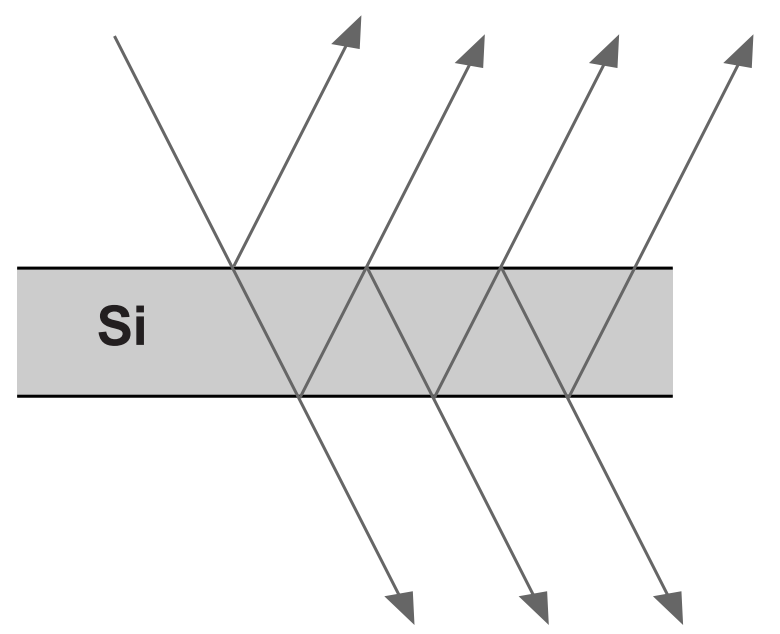

Figure 2.10: The beams reflected at the surfaces may interfere. This will modulate the reflected, transmitted and absorbed intensities. 

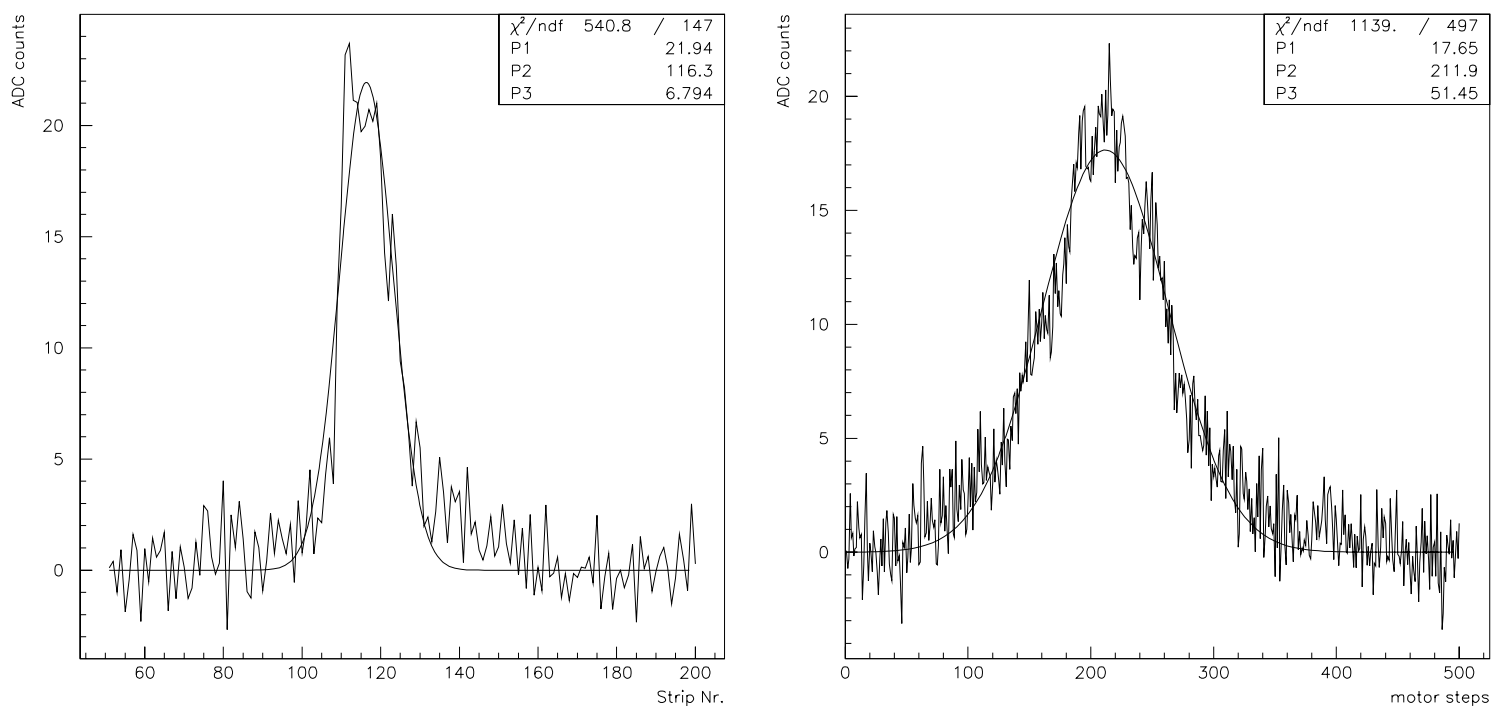

Figure 2.11: Beam profiles measured with a strip detector. The left profile is distorted due to interference effects. In the right plot the signal of a single strip is plotted while the laser beam is scanned across it. The height of the gaussian is a measure of the signal response of that strip to the laser light.

transmitted by the sensor, are a function of the silicon thickness. If the thickness of the sensor varies in the order of $\frac{\lambda}{4 n_{S} i}$ or more, the reflected intensity will be modulated, having maxima at positions of constructive and minima at positions of destructive interference. The same is true for the transmitted and absorbed intensities. This modulation of the absorbed light can cause a deformation of the beam profile [24]. Fig.2.11a shows such a distorted beam profile that was measured with the setup of fig.2.1. Again the pulsed Nd-YAG laser is coupled into a monomode fiber which ends in a collimator. In addition the detector module could be moved with a stepping motor, allowing scans perpendicular to the strips. The aim is now to measure the absorbed light as a function of the position on the sensor. In principle the signal height is a measure of the absorbed energy, but since the beam profile is very distorted by the interference, it is not possible to fit a gaussian curve to it. We therefore scan the beam across the strips and look at the output of one individual strip. The result is shown in fig. $2.11 \mathrm{~b}$ together with a gaussian fitted to the data. As can be seen, this curve is not distorted, and the maximal value can be used as a measure of the energy absorbed at this strip position. If this is done for every single strip on the sensor, and the values for the heights of the gaussians are plotted versus the strip positions, one obtains curves like the one shown in fig.2.12. We observe a very strong modulation with a factor of almost 3 between minima and maxima.

To show, that this pattern is caused mainly by the interference of the beams reflected on the front- and backside of the sensor, and not so much from the dielectric layer, a scan was performed on a detector before and after etching a hole into the backplane metallization. The result is shown in fig.2.13, where the dotted curve 


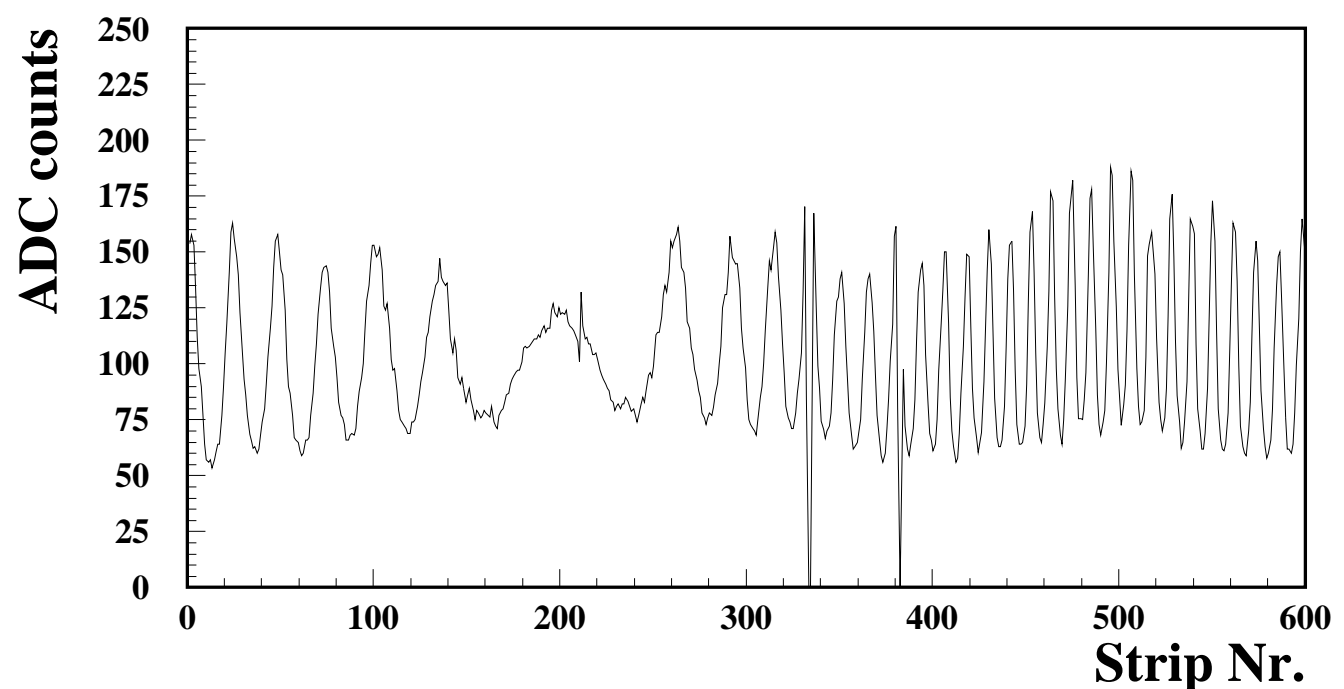

Figure 2.12: Signal response of the detector strips when a laser is scanned across the sensor. The modulation is an interference effect. The strips with zero response are bad channels.

indicates the measurement after the etching. In this measurements, the maxima in the pattern outside the hole region were normalized to each other. The hole on the backplane has three effects on the interference pattern:

- The average signal height is reduced, because part of the beam leaves through the backplane.

- The ratio between maxima and minima has changed to a factor of 2. This is due to the reduced reflectivity of the backplane.

- In the hole region the maxima have turned into minima and vice versa. This comes from the fact, that now the backside is an interface to a medium with smaller index of refraction (air). The phase factor between the incident and reflected wave changes by $180^{\circ}$ with respect to the reflection on the metal surface.

This is a clear indication, that the interference is dominated by the reflection at the sensor backside.

We will now take a closer look at the measured interference patterns. From these patterns one can obtain information on the variation of the sensor thickness. Fig.2.14 shows scans which were made on several sensors. The dark regions indicate absorption maxima, the light ones absorption minima. From the very regular patterns we can conclude, that the sensor thickness varies gradually over large distances, rather than being wavy. We also see, that the sensors are not very homogeneous in their thickness, and that they are generally flatter in the middle than at the edges. For the Nd-YAG wavelength of $1064 \mathrm{~nm}$, the difference in thickness between two adjacent maxima is $\frac{\lambda}{2 n_{S i}}=152 \mathrm{~nm}$. In fig. 2.14 for example we count 20 maxima from 


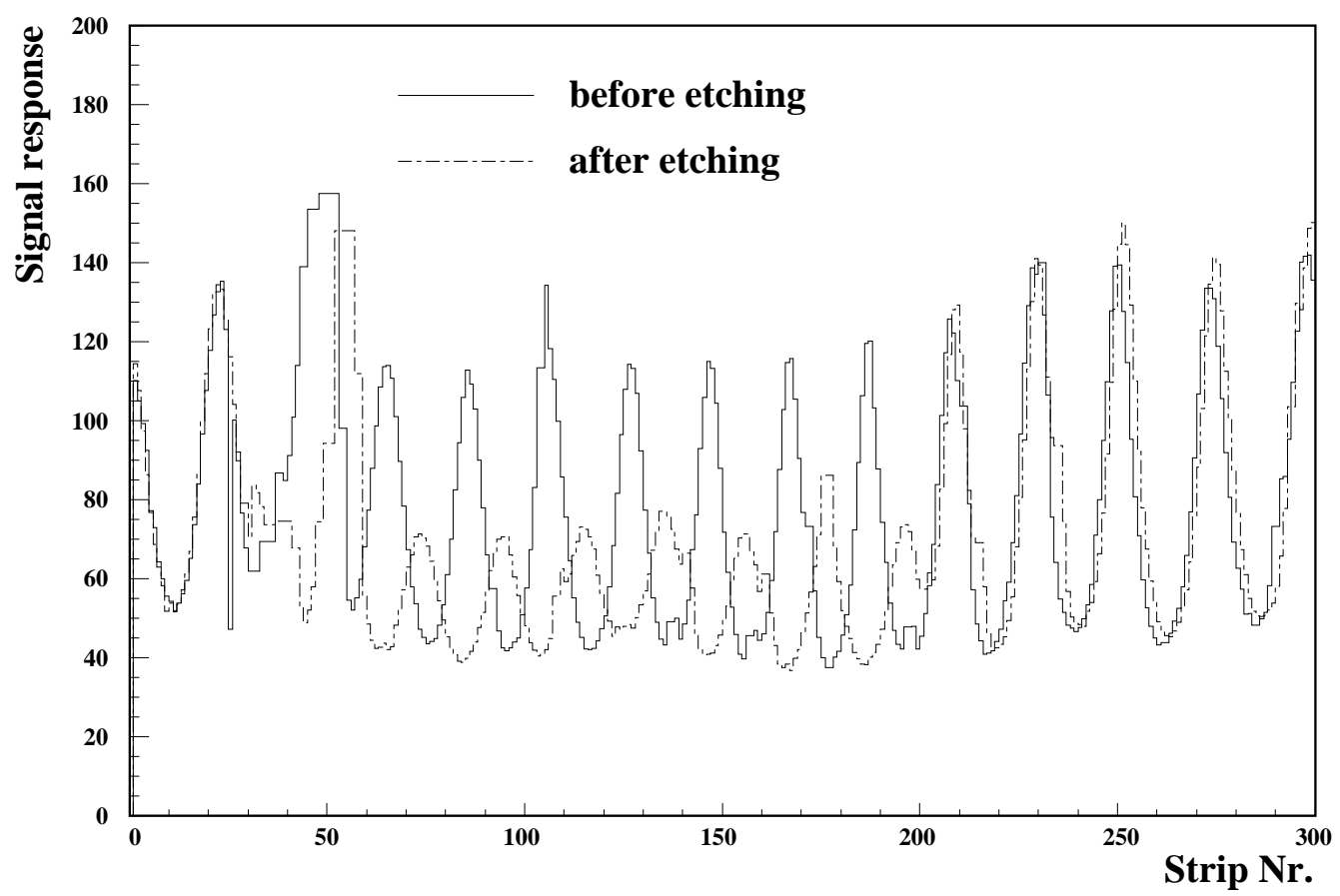

Figure 2.13: Interference pattern before and after etching a hole into the backplane.

the center to the sensor edge, which correspond to a change of $3 \mu \mathrm{m}$ in thickness. For all the measured sensors, the thickness variations stayed within the range of a few microns. These variations in sensor thickness are caused by the polishing and etching of the wafers during processing. A change of $76 \mathrm{~nm}$ in thickness already causes a change from maximal to minimal absorption. It is not realistic to get the sensor thickness so homogeneous that interference does not cause changes in the absorbed energy over an area of a few $\mathrm{cm}^{2}$. There are two ways to reduce the interference effects. The first is to cover the sensor with an antireflective coating. This would minimize the amount of reflected light and reduce the interference. One could also use a laser with a coherence length smaller than $2 \cdot d \cdot n_{S i}$ which corresponds to a spectral width of approximately $0.5 \mathrm{~nm}$. This is not achievable with the NdYAG laser. Laser diodes on the other hand can have spectral widths up to $2 \mathrm{~nm}$. Regarding minimization of the interference, such diodes would be better suited for the alignment system.

\subsection{Refraction of the beam}

If the incident laser beam is not perfectly perpendicular to the silicon surface, it will be refracted. If both silicon surfaces of the sensor are parallel, the ray will suffer just a parallel displacement, which for small angles of incidence $\alpha$ is given by:

$$
\delta=d \sin \alpha\left[1-\frac{n^{\prime}}{n}\right]
$$




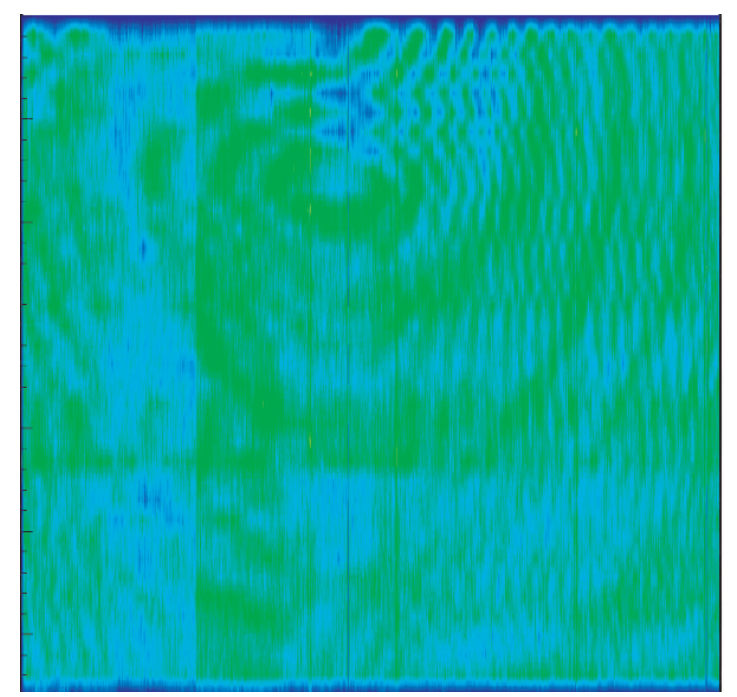

a

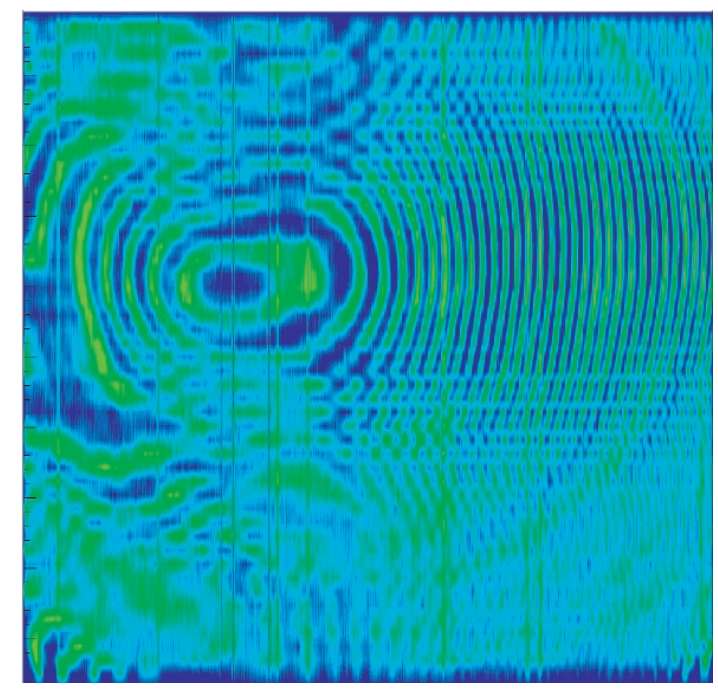

C

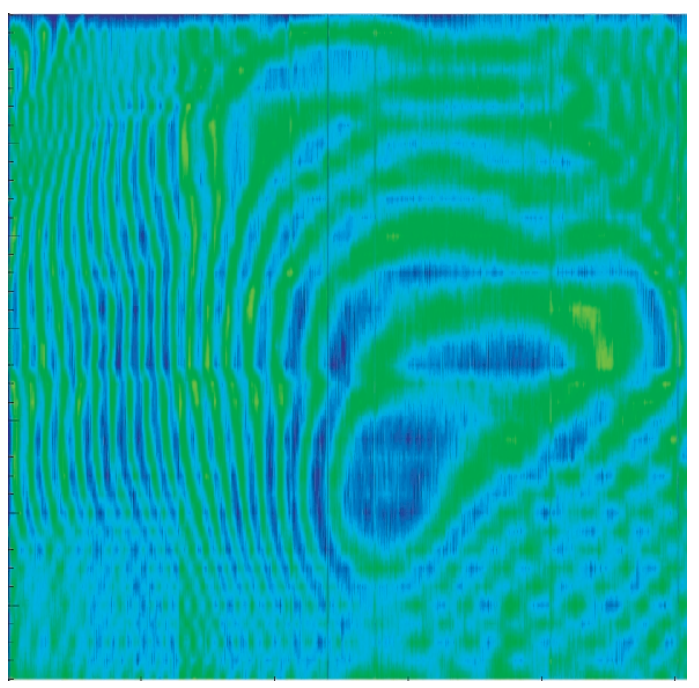

b

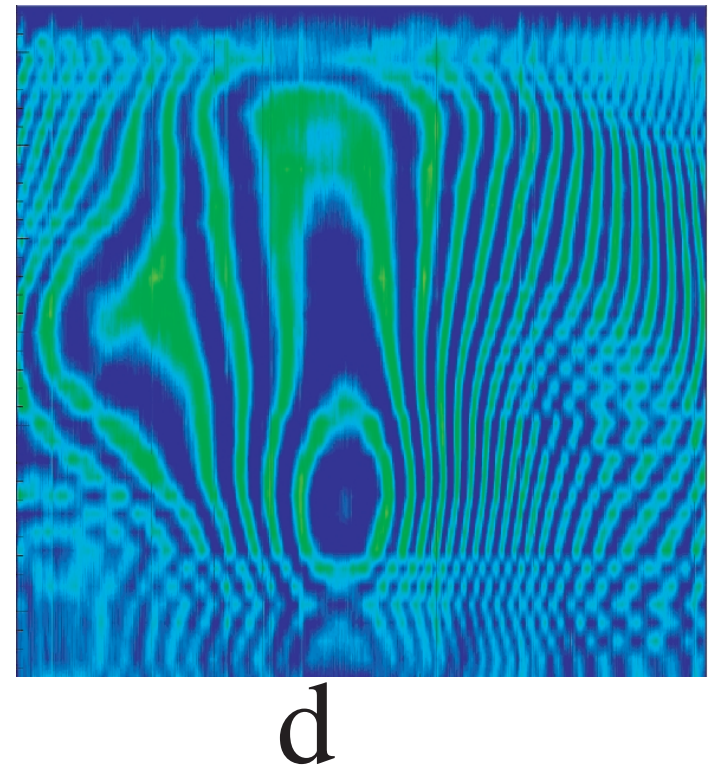

Figure 2.14: Interference patterns observed on various wafers. Dark regions correspond to absorption maxima, light regions to absorption minima. 
with $d=$ silicon thickness, and $n, n^{\prime}$ being the refractive indices of silicon and air respectively. With $n=3.5$ for silicon and $\alpha=1^{\circ}$ we obtain $\delta=3.7 \mu \mathrm{m}$. The mounting precision of the sensors will be far better than $1^{\circ}$, and we also do not expect any distortion or movement of this magnitude during operation. Any uncertainty in the beam position due to the parallel displacement can thus be neglected for our purposes. A tilt angle as foreseen for the mounting of the tracker barrel modules, can be accounted for in the calculations.

A more complicated situation is the case, where the two silicon surfaces are not perfectly parallel. The sensor then acts as a prism and changes the beam direction. As shown in sec. 2.3.4, the thickness of the sensor changes with position. The thickness profile can be inferred from the interference pattern. This is shown in figure 2.15 where the first plot shows an interference pattern measured on a strip sensor (see section 2.3.4), while the second shows the changes in thickness $\Delta y$ as a function of the position across the sensor. Note that neither the absolute thickness nor the sign of $\Delta y$ can be obtained from the measurement. $\Delta y$ was defined to be zero in the center region of the sensor, and the sign was arbitrarily set positive. The fact that the sensor thickness varies with position, means that the two surfaces form an angle $\epsilon$. The deviation angle $\Delta \alpha$, that the laser beam suffers by passing through the silicon wafer, is then given by

$$
\Delta \alpha=\epsilon-\alpha+\arcsin \left(\cos \epsilon \sin \alpha-\sin \epsilon \sqrt{n^{2}-\sin ^{2} \alpha}\right)
$$

To estimate the angle $\epsilon$, we need the distance $\Delta x$ between two interference maxima (or minima), which is found to be about $1.2 \mathrm{~mm}$. The path difference $\Delta$ in the silicon bulk between these two maxima is then $\Delta=\frac{\lambda}{n}$, yielding for the angle $\epsilon$ between the two surfaces:

$$
\tan \epsilon=\frac{\lambda}{2 n \cdot \Delta x}
$$

With the numbers given above we obtain values of $0.3 \mathrm{mrad}$ for the deviation angle $\Delta \alpha$, which corresponds to a transversal displacement of $30 \mu \mathrm{m}$ at a distance of $10 \mathrm{~cm}$.

\subsection{Accuracy in determining the laser spot posi- tion}

There are two effects, which affect the accuracy with which one can determine the position of the laser spot on the sensor:

- The accuracy of the fit to a perfect gaussian profile.

- Distortions in the gaussian profile.

The first effect can be minimized by choosing a beam diameter adapted to the sensor pitch and by using sufficient statistics as discussed in the next two subsections. Subsequently a closer look will be taken at deformations of the gaussian profile and their consequences on position accuracy. 

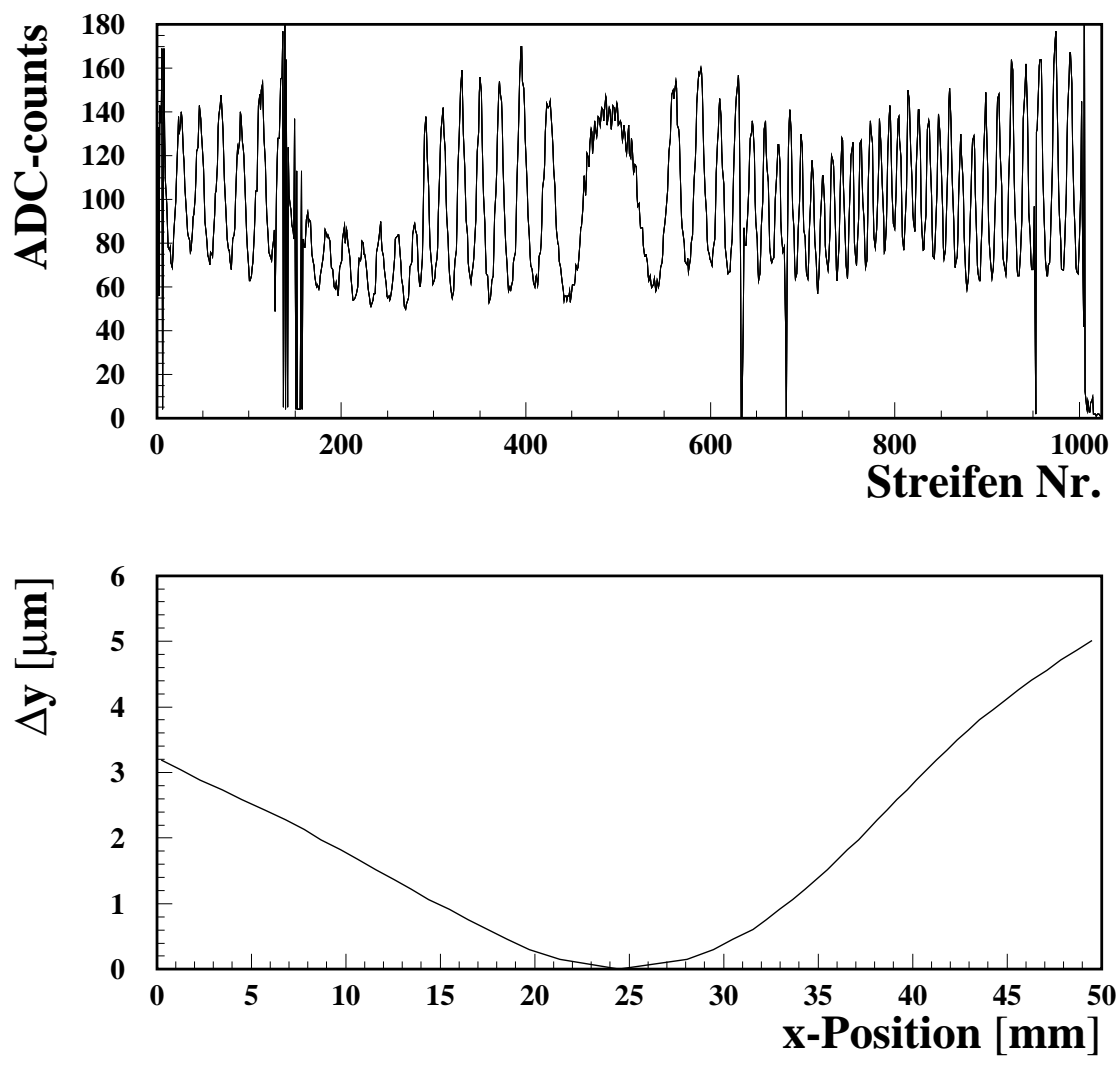

Figure 2.15: Interference pattern as measured on a strip detector, and the thickness change $\Delta y$ inferred hereof. Note that the sign of $\Delta y$ is ambiguous and has been arbitrarily chosen to be positive in this plot.

\subsubsection{Accuracy of the fit}

A simple calculation shows that for an ideal gaussian the position accuracy $\Delta m$ of the laser spot is a function of $\mathrm{S} / \mathrm{N}$ ratio, pitch $p$ and the width of the gaussian laser profile $\sigma_{L}$ (for a detailed derivation see appendix A):

$$
\Delta m=\frac{S}{N}^{-1} \cdot \sqrt{\frac{8}{3 \pi}} \sqrt{p \cdot \sigma_{L}}
$$

Here $\mathrm{S} / \mathrm{N}$ is defined as the signal at the maximum of the gaussian over the average strip noise. This result was derived for the case that the position of the laser spot is given by the center of gravity of the strip signals, but it is still a good approximation if a gaussian curve is fitted to the beam profile. As can be seen from eq.2.12, the accuracy gets worse with increasing pitch. The accuracy also deteriorates with increasing laser width $\sigma_{L}$. This is due to the fact that the maximum of the profile gets flatter and thus less well defined. Figure 2.16 shows a measurement of $\Delta m$ 
for several $\mathrm{S} / \mathrm{N}$ values. The solid line is the calculated precision from equation 2.12. The dashed line is a hyperbola fitted to the data of the gaussian fit. This measurement was performed by aiming the laser always on the same spot. Gaussian curves were fitted to several hundred pulses and their central values were filled into a histogram. The standard deviation of this distribution is $\Delta m$. In this way, possible deformations of the laser profile were always kept the same and did not affect the measurement of $\Delta m$. As one can see, the calculated curve fits very well to the COG measurement, for which it was derived. As one woul expect, the gaussian fit gives slightly better results.

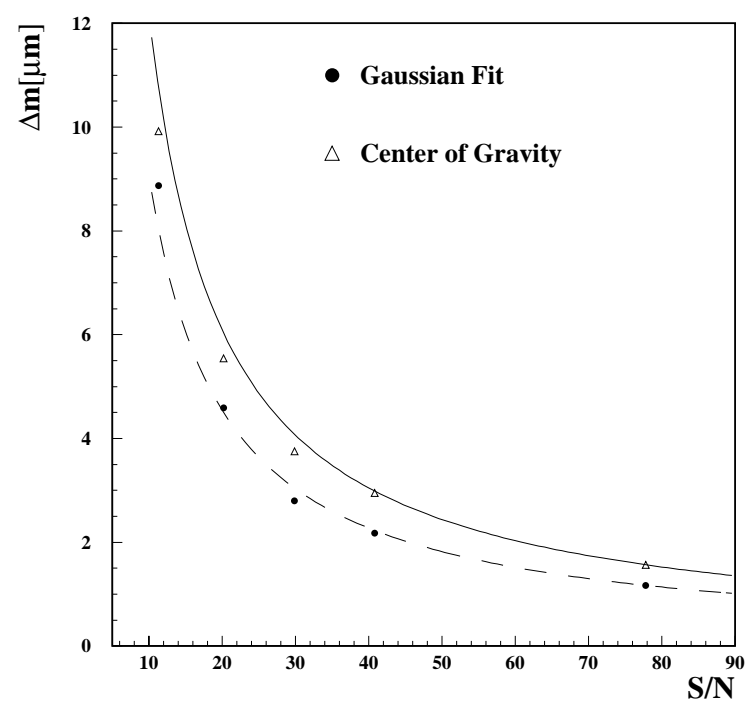

Figure 2.16: Measured accuracy for finding the laser spot as a function of $\mathrm{S} / \mathrm{N}$ ratio. The laser spot was kept at the same position, while the intesity was changed. The solid curve is the calculated precision, the dashed curve is a hyperbola fitted to the results of the gaussian fit.

In the setup that was used it was not possible to vary the width $\sigma_{L}$ of the laser beam. The effects of a change in $\sigma_{L}$ were asessed with the help of a simulation. In this simulation a gaussian profile with given width and height but random position is generated. Then gaussian noise is added to account for the electronics noise. The center of the profile is determined with a gaussian fit and the COG algorithm. The residual is then the difference between the reconstructed position and the position that was put in. As in the measurement, this was repeated several hundred times and the width of the residual distribution was taken as the precision $\Delta m$.

Figure 2.17 shows the simulated precision as function of $\sigma_{L}$ for a S/N-ratio of 10 . The curve is the calculation from eq.2.12. Again the gaussian fit gives slightly better results than the COG algorithm. The calculation doesn't fit the COG values as well as in the measurement presented before. In the calculation the assumption is made that the data points are distributed perfectly symmetrical around the center of the profile. In practice this can never be achieved due to the discrete strip positions 
and a continously varying laser spot position. In the measurement presented before however, this only causes a systematic shift in the reconstructed positions, since the laser spot did not move. $\Delta m$ in this case was the distribution width of the reconstructed positions. The systematic shift of the distribution does not affect its width. In the simulation the situation was different. Here the position of the profile was random and thus the shift in the reconstructed positions was also random and not systematic as in the measurement. This additional uncertainty causes the values of the simulated $\Delta m$ to be slightly higher than the calculated and the measured ones. These should also be the values taken for the design of the alignment system, since in practice the laser spot position can vary continously.

Note that in figure 2.17 the accuracy $\Delta m$ will not become infinetly small for very narrow beams. If the signal of the profile is confined to one single strip one would expect an accuracy $\Delta m=\frac{p}{\sqrt{12}}$ (i.e. the r.m.s. value of a flat distribution which is one strip wide).

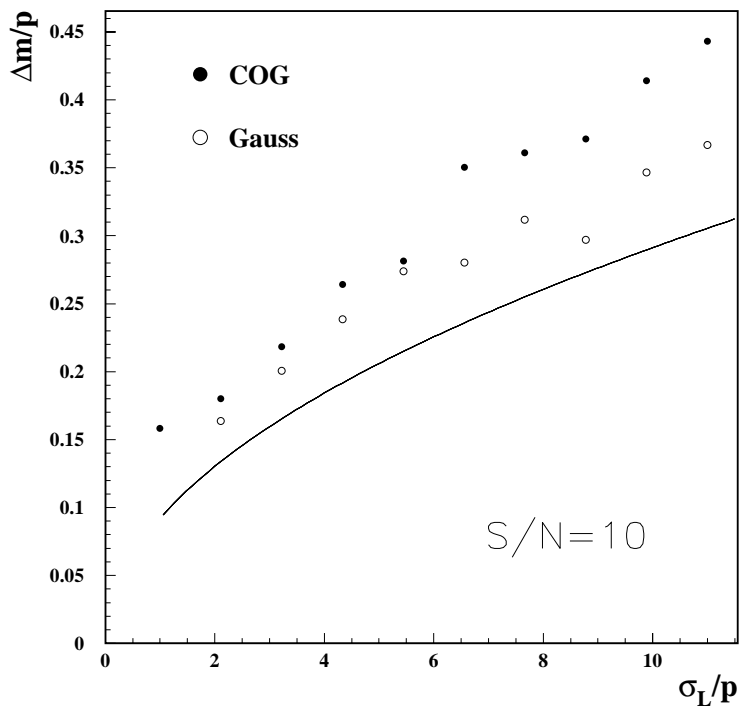

Figure 2.17: Simulation of the reconstruction of the laser spot position. The accuracy $\Delta m$ and the laser width $\sigma_{L}$ are given in pitch units. Results for a gaussian fit and the center of gravity algorithm are shown. The curve is the calculated precision (eq.2.12).

\subsubsection{Using statistics to improve $\mathrm{S} / \mathrm{N}$ ratio}

For an accurate determination of the position of the laser spot on a sensor, a sufficiently high $\mathrm{S} / \mathrm{N}$ ratio is needed. The $\mathrm{S} / \mathrm{N}$ ratio decreases for consecutive silicon layers, due to the absorption and reflection of the laser light. This loss in signal can be compensated to some extent, by sampling many pulses and adding them up as shown in figure 2.18. The left plot shows a single pulse sample measured with a strip sensor where the signal height is of the same order as the average noise 

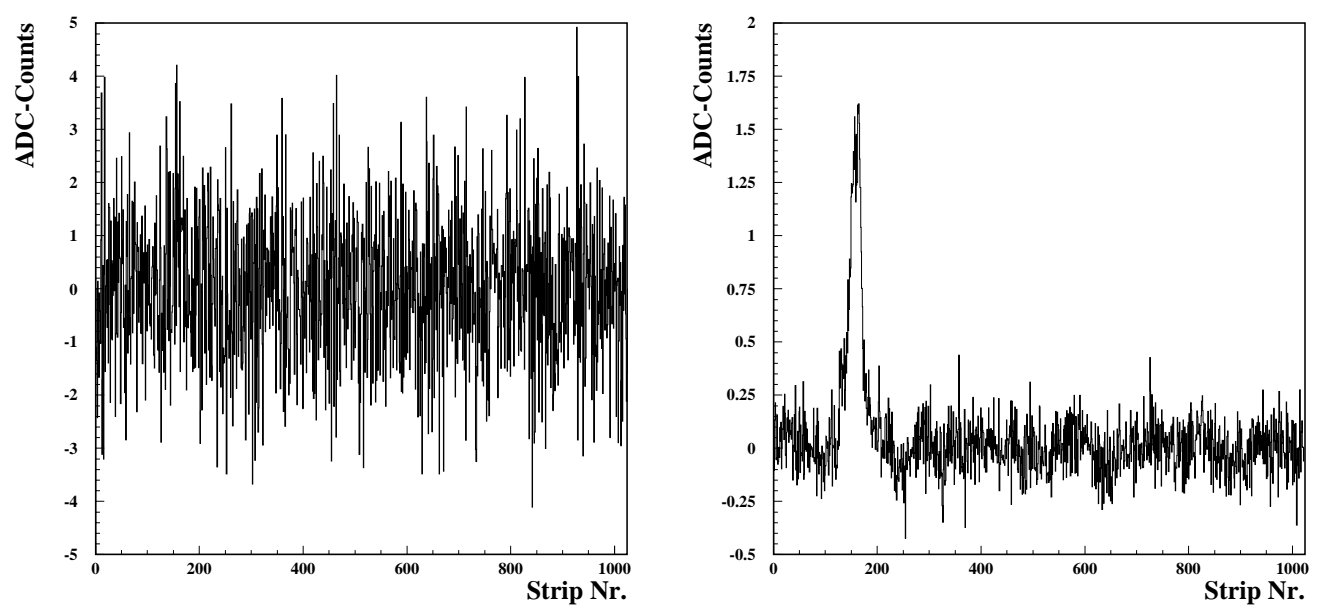

Figure 2.18: Comparison of a single pulse with $\mathrm{S} / \mathrm{N}=1$ and the average over 500 pulses.

$(\mathrm{S} / \mathrm{N}$-ratio $\approx 1)$. The beam profile is completely obliterated by the strip noise. The second plot shows the average over 500 pulses. As can be seen from the scale on the axes the noise has dropped dramatically and the signal is now clearly visible. Figure 2.19 shows the measured effective S/N-ratio as a function of the number of pulses over which the average is taken. The $\mathrm{S} / \mathrm{N}$ ratio of a single pulse is around 1 , while averaging over 500 pulses brings the effective $\mathrm{S} / \mathrm{N}$ ratio well above 10 . When adding up pulses, the signal should ideally increase linearly with the number of pulses $N_{\text {pulse }}$, while the noise goes with $\sqrt{N_{\text {pulse }}}$. This measured effective $\mathrm{S} / \mathrm{N}$ ratio does not follow exactly a square root function. It turns out, that there is a constant noise component, which does not decrease with statistics. This could originate from quantisation noise in the ADC.

\subsubsection{Effects of distorted profiles}

The effects of a distorted gaussian profile on the spot finding accuracy are hard to predict and have to be measured. For this purpose, the setup shown in figure 2.20 was used. The laser is mounted on a support which can be tilted, while the first silicon detector can be moved perpendicularly to the strips with a precision stage. The laser spot position is measured simultaneously on both sensors. In this way we have two degrees of freedom and two measurements, which makes it possible to reconstruct the tilt and the displacement. We will first study the two degrees of freedom separately and then show a combined measurement. In the following measurements the $\mathrm{S} / \mathrm{N}$ ratio was around 80 on the first layer and around 16 on the second. Additionally the average over a few hundred pulses was taken. In this way the effect of the $\mathrm{S} / \mathrm{N}$-ratio on the position accuracy becomes negligible. 


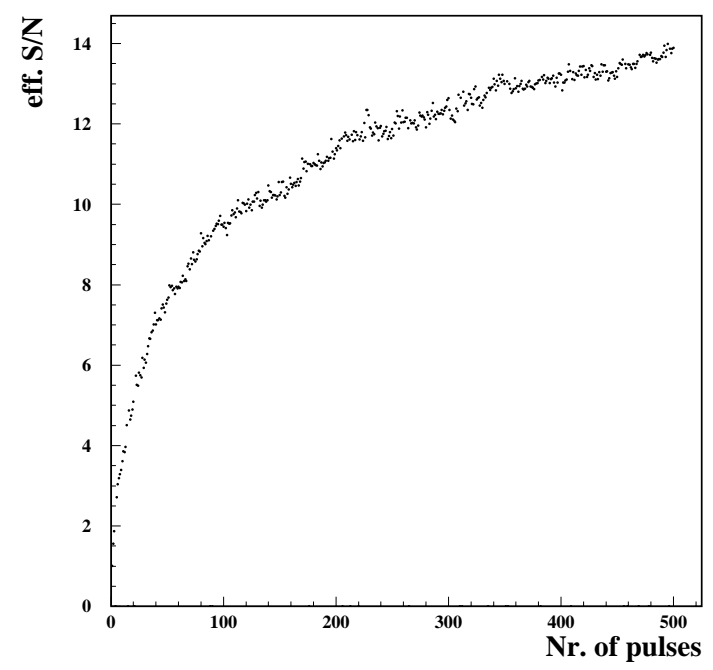

Figure 2.19: Effective signal to noise ratio versus the number of sampled pulses. The curve follows only in the beginning the expected square root function.

\section{Moving the first layer}

In this measurement the first sensor is scanned across the laser beam. The laser spot position is reconstructed only on the first detector by fitting a gaussian to the measured profile. Figure 2.21a shows the laser spot position plotted against the stage position for one scan across the sensor. After fitting a straight line to the data, we obtain for the slope the value of 1 . The distribution of the slope values obtained from various scans is shown in fig.2.21b. The mean value is equal to 1 with a precision better than 1 permille. The residuals are defined as the distance of the measured data points from the fitted straight line. Since the translation stage has a positioning accuracy around $5 \mu \mathrm{m}$, we can take the residuals as resulting almost entirely from the inaccuracies in determining the laser spot position. This measurement was repeated at various positions along the strips on the same sensor. The obtained residuals on the wafer are plotted on the left side in figure 2.22. This plot exhibits structures similar to the interference patterns discussed in section 2.3.4. This is an indication that the precision with which the laser spot position is measured, is affected by the distortions of the beam profile caused by the interference effects. The residuals should therefore be smaller in regions with smaller interference effects. Figure 2.23 shows a comparison of residuals measured in a region before and after a hole was etched into the backplane metallization. The residuals drop from peak values around $30 \mu \mathrm{m}$ with metallization to peak values around $10 \mu \mathrm{m}$ for the case when the hole is present. The plots on the right side in figure 2.22 show the distribution of the residuals. In the upper plot all the residuals that were measured are plotted. For the second plot the upper left and lower right quadrant of the sensor 

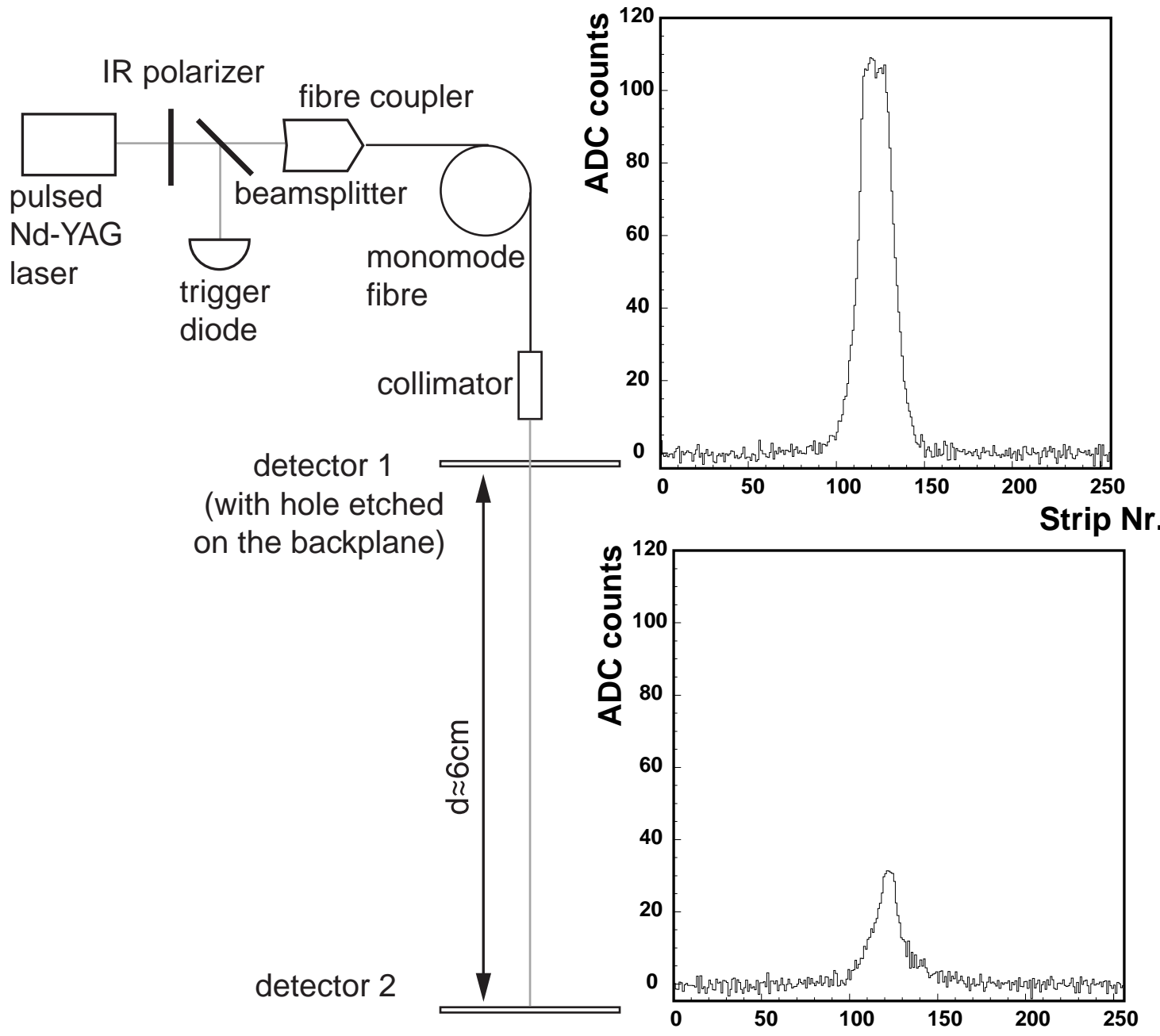

Strip Nr.

Figure 2.20: Setup used for determining the position accuracy of the laser spot. The support of the collimator can be tilted and the first detector can be moved with a precision translation stage. 

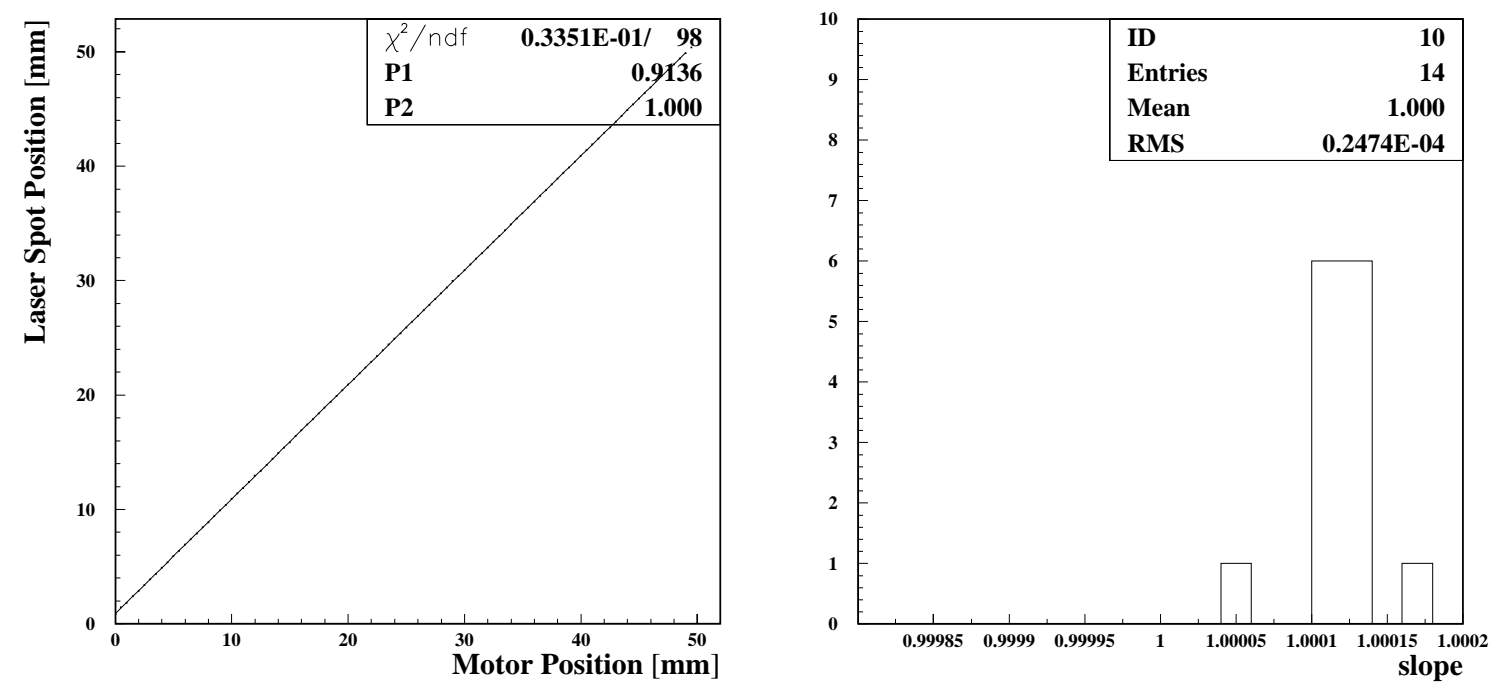

Figure 2.21: Left: Laser spot position plotted against the precision stage position for one scan. Right: Distribution of the slopes of the fitted straights for several scans.
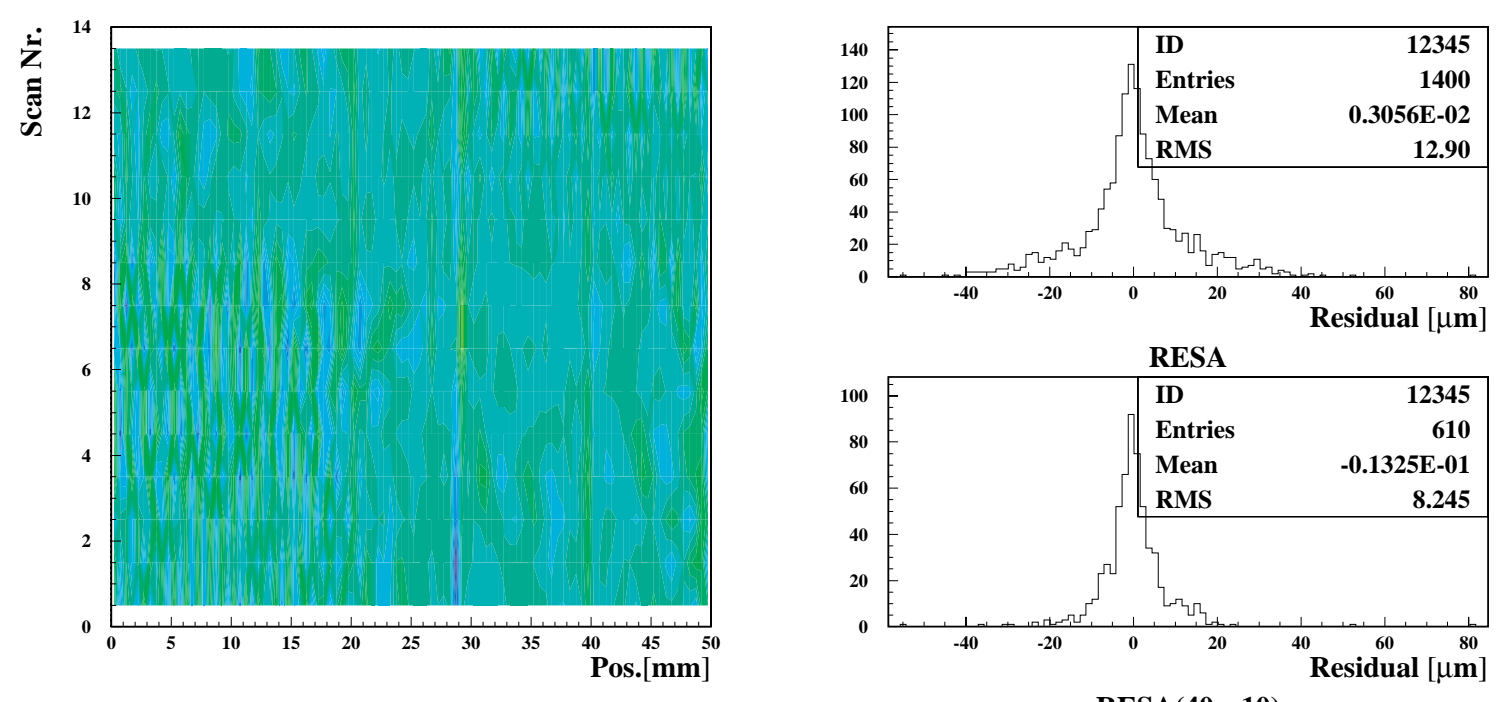

Figure 2.22: Left: Residuals measured on a wafer as function of the position. Light and dark areas correspond to $+50 \mu \mathrm{m}$ and $-50 \mu \mathrm{m}$. The observed structures are similar to the interference patterns. Top right: Distribution of the residuals on the whole wafer. Bottom right: Distribution of the residuals in upper left and lower right quadrant. 

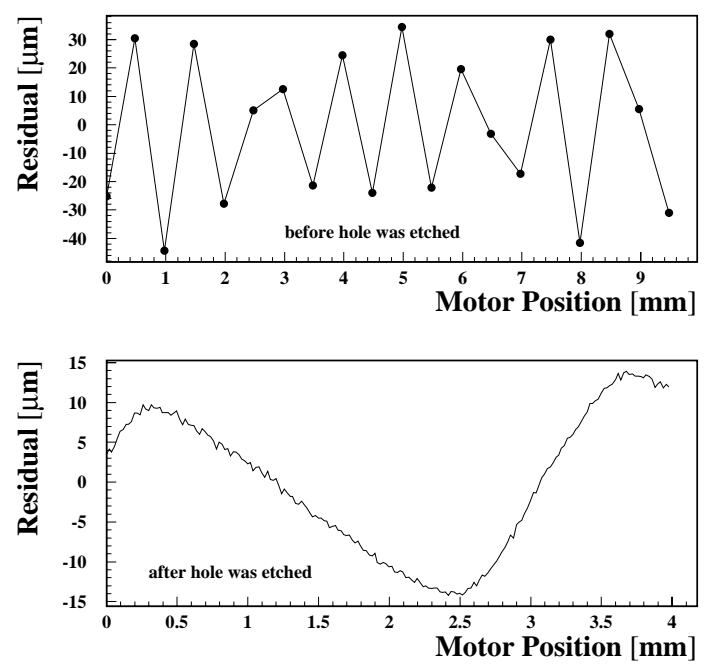

Figure 2.23: Residuals measured in the same sensor region before and after the hole in the backplane was etched.

were taken, where the interference is less strong. Over the whole module the rms of the residuals is about $13 \mu \mathrm{m}$ with tails to larger values. In the regions with less interference, the rms of the residuals becomes less than $10 \mu \mathrm{m}$.

We now examine the residuals on the second layer. Here the position should always stay the same while the first layer is moved. The residual is defined as the deviation of the laser spot from its average position. The measured residuals for the second layer are shown in figure 2.24. In the first plot the residual and the beam intensity are shown as function of the position of the precision stage which moves layer 1 . The intensity rises sharply as the hole moves into the beam region, then stays roughly constant and drops again sharply as the hole moves away from the laser beam. The observed residuals are largest at the edges of the hole, since here the beam is partially shadowed by the backplane metalisation causing a strong distortion of the beam profile. In the central region the residuals still display a systematic change coming from the distortion of the profile due to interference in the first layer. The distribution of the residuals is shown in figure 2.24b. For the whole region over which the intensity is constant $(2-8 \mathrm{~mm})$, the residuals stay within $25 \mu \mathrm{m}$. Since in the final system we expect the laser beam to wander at most a millimeter or so on a module, the residuals of a smaller region (4-6 mm) are also shown in the plot. For this region the residuals stay within $10 \mu \mathrm{m}$.

\section{Tilting the beam}

When the laser beam is tilted a correlated displacement of the beam spots on both sensors is observed. A measurement is shown in figure 2.25 on the left. $\Delta m$ is the 

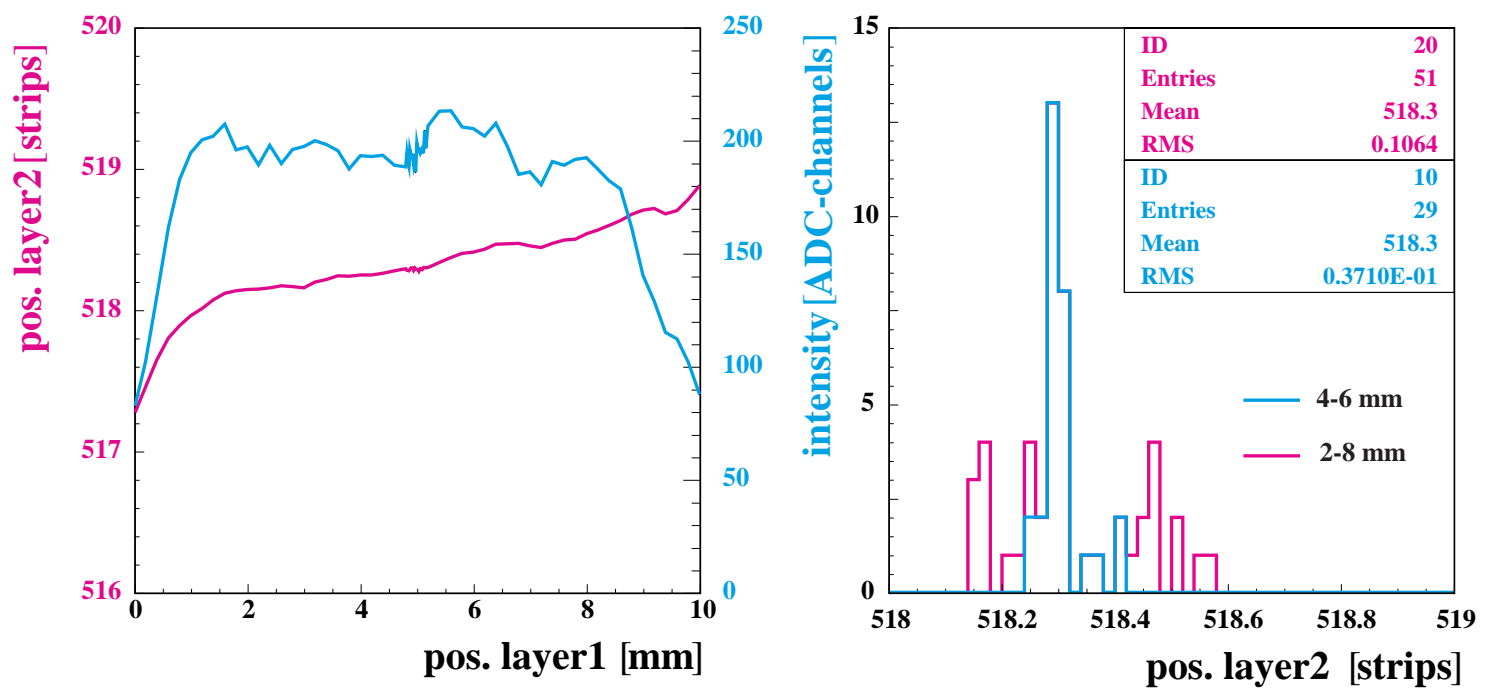

Figure 2.24: Residuals measured on the second layer while the first one is displaced. The pitch of the sensors is $50 \mu \mathrm{m}$.

setting of the micrometer screw which tilts the collimator support as shown in the insert on the bottom right. Straight lines were fitted to the laser spot positions and again the deviation of the measured position from the fitted straight line was taken as the residual. The distribution of the residuals on both layers is shown on th right side of the figure. The RMS of the residuals is about 0.2 in strip units. With a pitch of $50 \mu \mathrm{m}$, this corresponds to $10 \mu \mathrm{m}$. This is consistent with the measurements shown above.

\section{Combined measurement}

Finally a combined measurement was performed, in which the beam was tilted simultaneously with a displacement of the first sensor. To avoid any systematic effects a random walk was generated for the position of the precision stage and the tilt screw setting. With the straight line fits obtained above from tilting the beam (fig.2.25), it is possible to reconstruct the screw setting $\Delta m$ and the position of the first sensor from the measured laser spot positions. The result is displayed in figure 2.26. In the first plot the curves are the random settings, while the symbols are the reconstructed values. The residuals are defined as the difference between the two and their distribution is shown in the second plot. For the position of the precision stage we get an RMS of $13 \mu \mathrm{m}$. The distribution of the reconstructed screw setting has an RMS of $3.6 \mu \mathrm{m}$, which corresponds to a displacement of $10 \mu \mathrm{m}$ on the second sensor. These results are consistent with the measurements shown above.

Summarizing we can say, that the laser spot can be detected on the modules with an accuracy around $10 \mu \mathrm{m}$. This result holds for $50 \mu \mathrm{m}$ pitch modules, a 

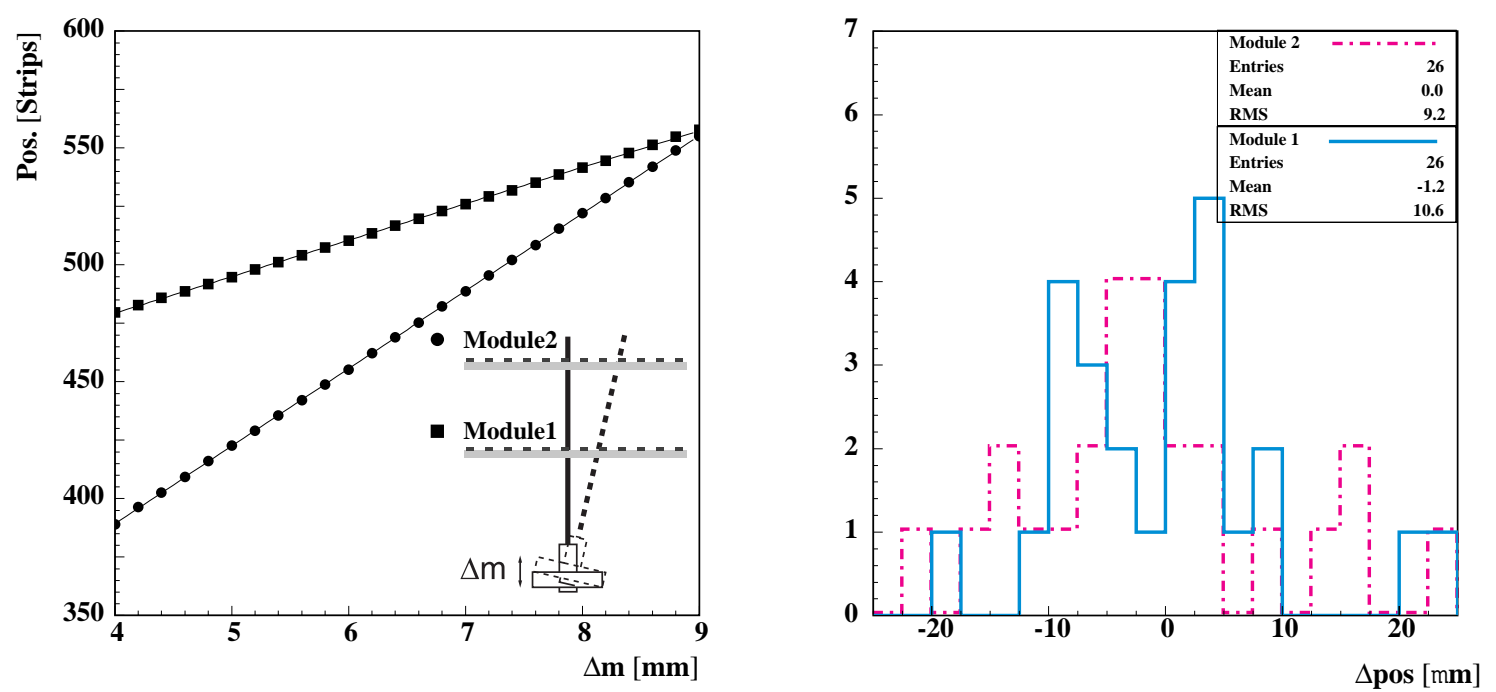

Figure 2.25: The beam can be tilted with a precision screw. $\Delta m$ is the screw setting. The plot on the left shows the measured laser spot positions as function of the screw setting. The histogram on the right shows the distributions of the residuals on both modules.

beam diameter of $1.5 \mathrm{~mm}$ and a $\mathrm{S} / \mathrm{N}$-ratio large enough to neglect the influence on the fit accuracy $\Delta m_{f i t}$. For different pitches, beam diameters and $\mathrm{S} / \mathrm{N}$-ratios this figure has to be rescaled. The uncertainty $\Delta m_{\text {dis }}$ due to the profile distortions scales directly with laser width $\sigma_{L} . \Delta m_{\text {fit }}$ has to be rescaled according to section 2.5.1 (eq.2.12). 

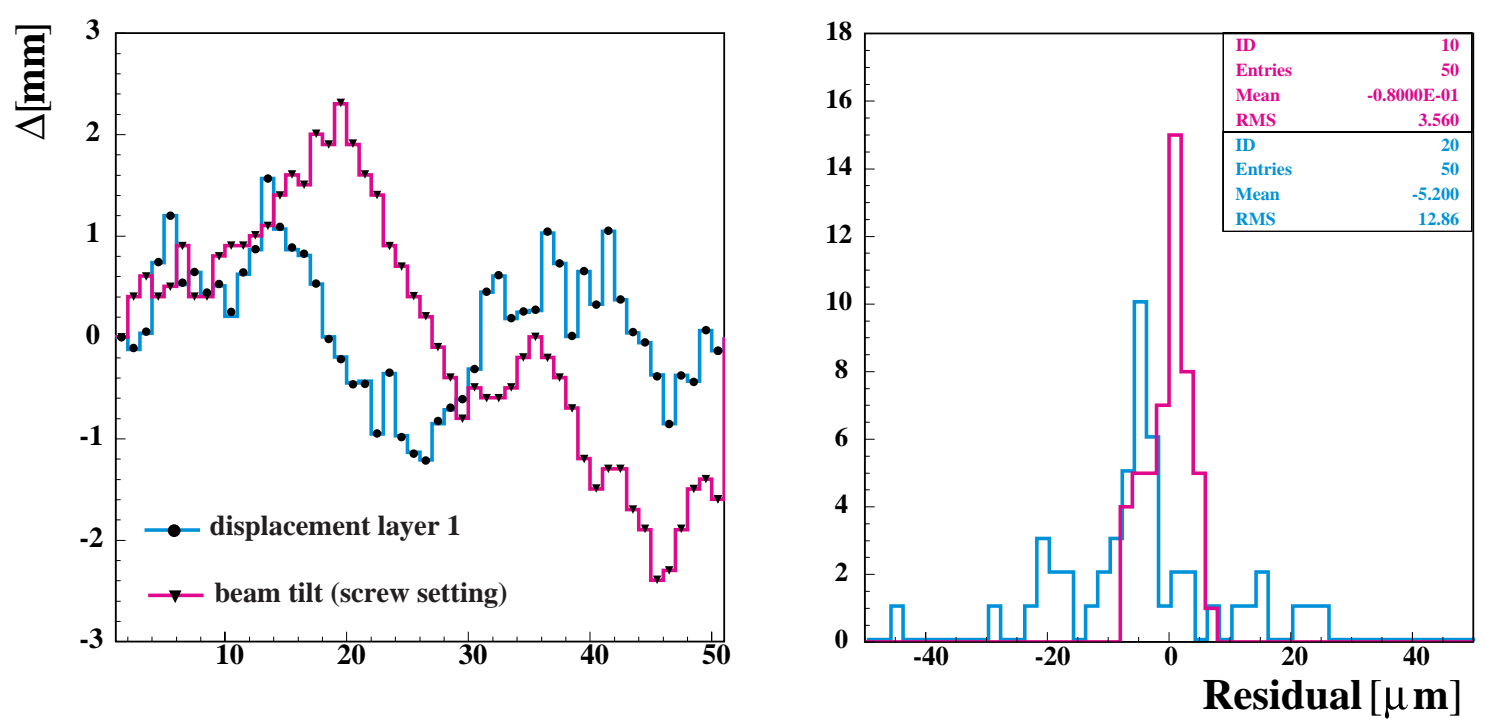

Figure 2.26: Measurement in which a displacement was combined with the beam tilt. The curves on the left are the randomly generated settings while the symbols are the measured positions. On the right the histograms with the residual distributions are shown. 


\section{Chapter 3}

\section{The CMS Laser Alignment System}

\subsection{Layout}

\subsubsection{Number and Position of Beams}

Fig.3.1 shows the laser alignment system that has been proposed for the CMS tracker [25]. There are two sets of beams (rays 2 and 3), which align the endcap disks with respect to each other. These rays hit all the 9 disks and have an 8 -fold azimuthal symmetry. The fibers enter the endcap at the end flange and run along the outer rim of the disks to disk \#5. They then enter in-between the disks and run down to rings 7 and 4, ending in a collimator to which a prism is attached. This prism sends out two back-to-back beams forming rays 2 and 3. For the barrel there are no such rays which hit more than one layer. This is due to the lack of space in the barrel region. This means, that for the relative alignment of the layers the barrel has to rely on the mechanical rigidity of its support structures and on alignment with tracks. The laser alignment system foresees a set of beams (ray 4), to align the two endcaps with respect to each other and with respect to the inner and outer barrel. Here the fibers enter the tracker in-between the endcap and the barrel region. Again a collimator/prism combination generates two back to back beams. The beams oriented toward the endcaps hit disks \#1-3 at the same radius as ray 3 . The beams in the opposite direction pass between inner and outer barrel. Beam splitters are arranged along these rays, reflecting them toward inner and outer barrel. In this way the relative movements of endcaps, inner and outer barrel are seen by several sensors and can be reconstructed. The $\phi$-positions of these rays are given by the module arrangement in the outermost layer of the inner barrel and the innermost layer of the outer barrel and are summarized in table 3.1. The purpose of the last set of rays (Nr.1) shown in fig.3.1 is to measure the relative displacements between the muon chambers and the tracker. Due to the 6-fold symmetry of the muon system there are 6 laser beams on each side. Laser boxes that are attached to the support structure of the electromagnetic calorimeter emit pairs of laser beams toward the tracker and the muon system. In the tracker these beams hit 3 layers of silicon sensors in the endcaps. The position and the orientation of these beams is carefully 


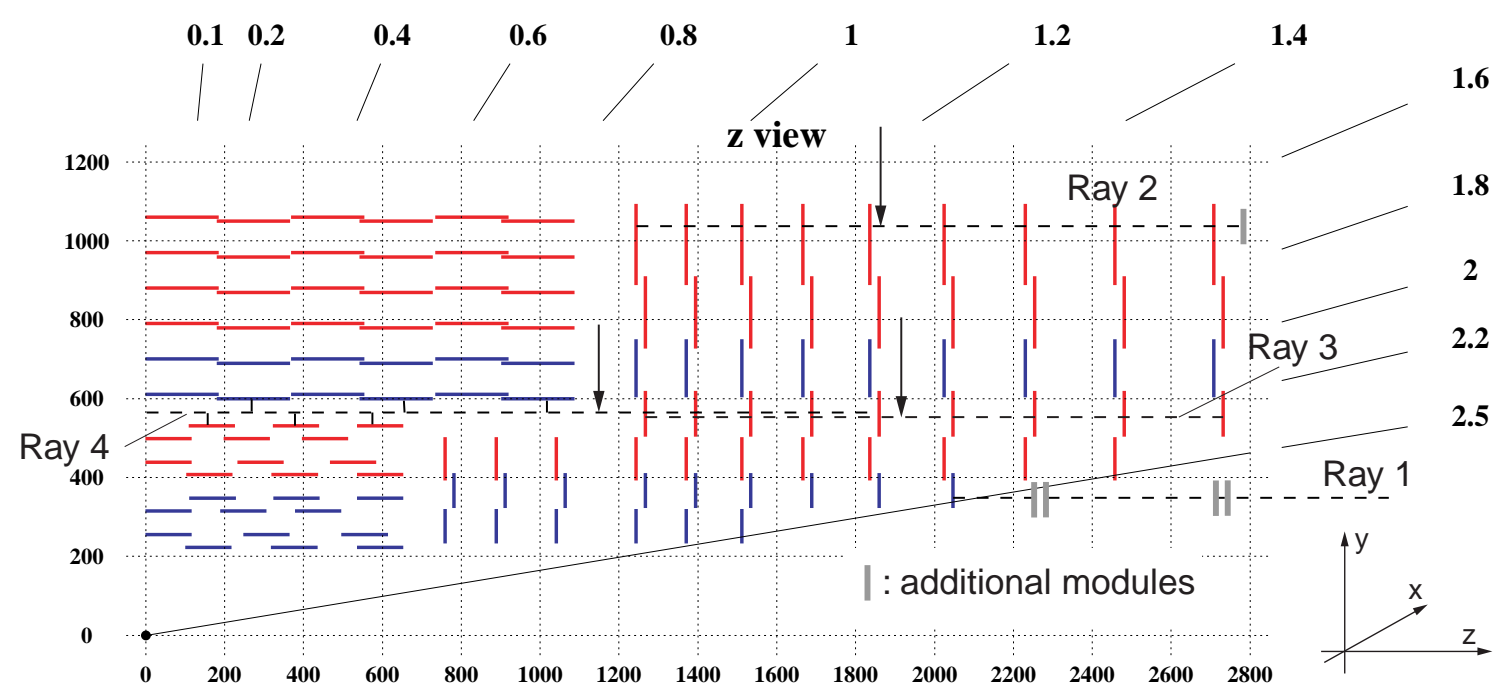

Figure 3.1: Layout of the laser alignment system. The arrows indicate the positions where the fibers run radially inwards.

\begin{tabular}{|l|l|l|}
\hline Channel & $\phi$ position $[\mathrm{rad}]$ & $\phi$ position $[\mathrm{deg}]$ \\
\hline \hline 1 & 0.39 & 22.5 \\
\hline 2 & 1.29 & 73.9 \\
\hline 3 & 1.85 & 106.1 \\
\hline 4 & 2.75 & 157.5 \\
\hline 5 & 3.65 & 208.9 \\
\hline 6 & 4.32 & 247.5 \\
\hline 7 & 5.22 & 298.9 \\
\hline 8 & 5.78 & 331.1 \\
\hline
\end{tabular}

Table 3.1: $\phi$-position of the 8 laser beams of ray 4 . 
measured in the endcaps and in the muon system. In this way the tracker can be aligned with respect to the muon detectors.

Putting all numbers together we have $2 \times 8$ beams for rays 2 and 3,8 beams for ray 4 and $2 \times 6$ beams for ray 1 , adding up to a total of 52 laser beams for the alignment system.

The coordinate system commonly used for CMS is sketched in figure 3.1. It is a right-handed system with the y-axis pointing upward, the $\mathrm{x}$-axis toward the center of the LHC ring and the z-axis along the LHC beam direction. In this frame, the alignment rays 2,3 and 4 all point in the z-direction. They only hit single sided modules and are thus only capable to detect the displacement of the laser spot in the $\phi$-direction. For the alignment of the endcap disks with respect to each other, it will be possible to detect rotations around the $\mathrm{z}$-axis and displacements along $\mathrm{x}$ and $\mathrm{y}$ with the help of rays 2 and 3 . For these rays, rotations around the $\mathrm{x}$ - and $\mathrm{y}$-axis will only be visible in second order, and displacements in z will not be seen at all.

In principle it would be possible to detect also movements in $\mathrm{z}$ and rotations about $\mathrm{x}$ and $\mathrm{y}$, by using laser beams which are not parallel to the z-axis. This would be a severe complication for the alignment system, since it is difficult to find paths for the laser beams, which are not obstructed by support structures, electronic boards or services. Beams running parallel to the z-axis are easier to manage, because all the disks have identical support structures and services. Once an opening for a beam is foreseen in one place, the beam can pass through all 9 disks. For beams which are not pointing in $\mathrm{z}$, the openings would have to be at different positions on each disk. Since $\mathrm{n}$ the accuracy needed for tracking the $\mathrm{z}$-coordinate is the less critical one, these degrees of freedom (rotations about $\mathrm{x}$ and $\mathrm{y}$ and displacements in $\mathrm{z}$ ) will not be considered in the following.

Ray 4, used to monitor relative movements of the endcaps and the barrels with respect to each other, can measure displacements in $\mathrm{x}$ and $\mathrm{y}$ and rotations around all three axes.

\subsubsection{Nd-YAG Laser / Fiber Coupling}

To estimate the pulse energy needed for each beam, we look at a stack made of 5 layers of silicon sensors. We require an effective S/N-ratio of 10 on the last layer. As shown in section 2.5.2 a S/N-rato of 1 for a single pulse is enough to reach the $\mathrm{S} / \mathrm{N}$-ratio of 10 by averaging over a few hundred pulses.

The largest pulse power required on the first layer is then $60 \mathrm{pJ}$, and since each beam is split in two, a power of $120 \mathrm{pJ}$ per beam is required. The pulse power of the Nd-YAG laser is larger than $3 \mu \mathrm{J}$, so it is possible to generate many alignment beams with one laser. We consider using an optical hub which splits the Nd-YAG laser beam into 32 secondary beams that will be coupled into monomode fibers. Two of these hubs would be used to generate the 52 beams needed for the alignment system leaving 12 spare beams. This scenario allows us to have losses of a factor up to 800 in the splitters, fiber couplers and collimators. This is a lot of reserve, since coupling efficiencies of $30 \%$ are easily achievable with monomode fibers and losses 
in beamsplitters and lenses are negligible. The main difficulty lies in achieving a homogeneous intensity for all the beams. The fibers leaving the hub are routed into the tracker volume, where they are collimated and split as described in 3.1.1.

\subsection{Performance Parameters}

The ideal case for the laser alignment system would be if the laser beams were perfectly straight, and the intersection points of these straight lines with the disks could be measured with infinetely high precision. In the real system, we expect the beams to have kinks due to refraction, as discussed in section 2.4. Furthermore the accuracy in determining the intersection points with the disks is limited by the precision in finding the laser spot on a detector module and by the uncertainties with which the modules are located on the mechanical structure. In this section we will discuss all these aspects and finally estimate the precision, with which one can find the intersection points of the beams with the disks.

\subsubsection{Refraction}

In principle it is possible to measure the refraction for each module as a function of the position where the beam passes through a module. Then one could account for the refraction in the reconstruction of the beams. Since this would be very complicated, it is better to make sure that the refraction is small enough to be neglected without destroying the required accuracy of the system. We therefore will consider refraction to be an uncertainty factor and treat it as if the beams were scattered in each sensor. We consider a set of sensors which measure a traversing beam as sketched in fig.3.2. In each sensor the beam suffers a random deviation angle

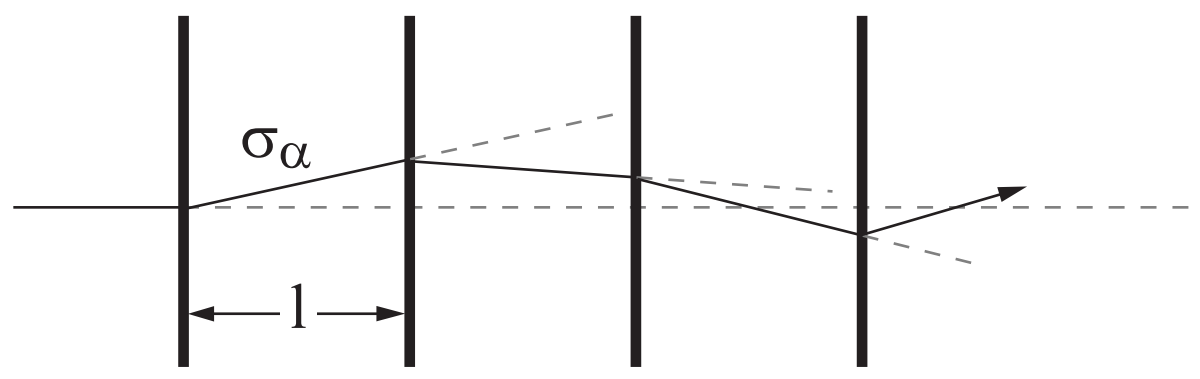

Figure 3.2: Refraction is considered to be an uncertainty and is treated similar to multiple scattering of a particle track.

$\Delta \alpha$ described by a distribution width $\sigma_{\alpha}$. If we look at the deviation of the measured $\mathrm{x}$-positions from the nominal beam position, we easily see, that the deviation grows as one moves toward the last plane. The $n$-th sensor measures a deviation with $\sigma_{x n}=\sigma_{\alpha} \sqrt{\sum_{i=1}^{n-1} l_{i n}^{2}}$, where $l_{i n}$ is the distance between the $i$-th and the $n$-th plane. If however we just fit a straight line through the measured points and then ask how much the points deviate from this straight line, we find that $\sigma_{x}=\frac{l \cdot \sigma_{\alpha}}{\sqrt{6}}$, where 1 is 
the distance between two consecutive planes. This result is valid for small incidence angles and equidistant planes.

In section 3.3 we will see, that the alignment system will just rely on the fact that the laser beams are straight lines, independently of small deviations in their orientation. This justifies the approach described above and we will take $\sigma_{\alpha}=\frac{\Delta \alpha_{\max }}{\sqrt{12}}$, with $\Delta \alpha_{\max }=0.3 \mathrm{mrad}$ as calculated in sect. 2.4 .

\subsubsection{Laser spot accuracy}

In this section we will apply the results from section 2.5 to the modules of the proposed alignment system, and obtain values for the accuracy $\Delta m_{\text {spot }}$ with which the laser spot can be found on a single detector module. This uncertainty is a sum of the contribution from the fitting accuracy and from the distortion of the gaussian profile:

$$
\Delta m_{\text {spot }}=\sqrt{\Delta m_{\text {fit }}^{2}+\Delta m_{\text {dis }}^{2}}
$$

The modules which will be used for the laser alignment system have a larger pitch than the ones which were used in the measurements of section 2.5. Also in the final system the beam diameter may be different. We therefore have to rescale the values obtained for $\Delta m_{\text {dis }}$ with the laser beam width and the fit accuracy $\Delta m_{f i t}$ according to eq.2.12:

$$
\Delta m_{f i t} \propto \frac{S}{N}^{-1} \cdot \sqrt{p \cdot \sigma_{L}}
$$

The results for the different modules are summarized in table 3.2. Two different beam diameters have been considered: $\sigma_{L}=0.25 \mathrm{~mm}$ and $\sigma_{L}=1 \mathrm{~mm}$. The uncertainty coming from the profile distortions is then $7 \mu \mathrm{m}$ and $29 \mu \mathrm{m}$ respectively. The values were calculated for a S/N-ratio of 10 . As can be seen, even for a very wide laser beam and the largest pitch the accuracy with which the laser spot can be found on the module is better than $50 \mu \mathrm{m}$. For the smaller beam diameter it gets below

\begin{tabular}{|c|c|c|c|c|c|c|}
\hline \multirow[t]{2}{*}{ Ray Nr. } & \multirow[t]{2}{*}{ Module } & \multirow[t]{2}{*}{ Pitch $[\mu \mathrm{m}$} & \multicolumn{2}{|c|}{$\sigma_{L}=0.25 \mathrm{~mm}$} & \multicolumn{2}{|c|}{$\sigma_{L}=1 \mathrm{~mm}$} \\
\hline & & & $\begin{array}{l}\Delta m_{f i t} \\
{[\mu \mathrm{m}]}\end{array}$ & $\begin{array}{l}\Delta m_{\text {spot }} \\
{[\mu \mathrm{m}]}\end{array}$ & $\begin{array}{l}\Delta m_{f i t} \\
{[\mu \mathrm{m}]}\end{array}$ & $\begin{array}{l}\Delta m_{\text {spot }} \\
{[\mu \mathrm{m}]}\end{array}$ \\
\hline 1 & TEC Ring 2 & 128 & 16 & 17 & 33 & 44 \\
\hline 2 & TEC Ring 7 & 156 & 18 & 19 & 36 & 46 \\
\hline $3 / 4$ & TEC Ring 4 & 120 & 16 & 17 & 32 & 43 \\
\hline 4 & TIB 2 & 120 & 16 & 17 & 32 & 43 \\
\hline 4 & TOB 1 & 122 & 16 & 17 & 32 & 43 \\
\hline
\end{tabular}
$20 \mu \mathrm{m}$.

Table 3.2: Expected accuracy for finding the position of the laser spot on the modules used in the laser alignment system. A S/N-ratio of 10 was assumed and the values are given for $\sigma_{L}=0.25 \mathrm{~mm}$ and $\sigma_{L}=1 \mathrm{~mm}$. 


\subsubsection{Overal Accuracy}

The reconstruction of the deformations by the alignment system starts from the measured intersection points between the laser beams and the components whose movement is to be monitored. For rays 2 and 3 in figure 3.1 these components are the endcap disks, while for ray 4 they are the endcap disks, the outermost layer of the inner and the innermost layer of the outer barrel. The accuaracy with which these intersection points can be measured depends on the laser spot accuracy $\Delta m_{\text {spot }}$, the uncertainty $\Delta m_{r e f r}$ introduced by kinks in the beam due to refraction, and the precision $\Delta m_{\text {mech }}$ with which the position of a strip sensor is known with respect to the support structure.

There are two kinds of measurements that we will consider in the following, namely absolute measurements, where position and orientation of the components with respect to each other are measured, and relative measurements, where only the changes of these parameters are monitored. For the precision of absolute measurements all the contributions from above add up: $\Delta m=\Delta m_{\text {spot }} \oplus \Delta m_{\text {refr }} \oplus \Delta m_{\text {mech }}$. In the relative measurements only $\Delta m_{\text {spot }}$ is of importance, since the positions of the modules with respect to the mechanical structure and the kinks in the beams due to refraction are not expected to change significantly from measurement to measurement. For the mechanical precision two scenarios are conceivable: First one could rely only on the assembly precision which is around $50 \mu \mathrm{m}$. In the second case, the position of the modules on the structures is measured after assembly with a coordinate measuring machine. In this case a precision $\Delta m_{\text {mech }} \approx 20 \mu \mathrm{m}$ can be reached. For the beams which are used for the internal endcap alignment all these uncertainties are summarized in table 3.3 for a beam diameter $\sigma_{L}=0.25 \mathrm{~mm}$. The numbers for rays 2 and 3 differ slightly. This is due to the modules in ring 7 having larger pitch than those in ring 4 , thus resulting in different values of $\Delta m_{f i t}$. The accuracy for the absolute measurement without metrology is dominated by the mechanical mounting accuracy. With metrology the contribution due to refraction becomes important. In the relative measurement the uncertainties in finding the laser spot on the module is the only contribution.

\subsection{Reconstruction}

The purpose of the laser alignment system is to detect movements and deformations of the mechanical structure, which are inferred from the position of the laser spots on the strip sensors. In this section we shall describe, how this is done for the internal alignment of the endcaps with rays \#2 and \#3. The alignment of the four tracker subdetectors, namely inner and outer barrel and the two endcaps, with ray \#4 can be performed in an analogous way. A description of the alignment of the tracker with respect to the muon chambers using ray 1 can be found in [26].

The first step is to parametrize the movements and deformations. For the alignment of the 9 endcap disks one obtains 49 parameters to which we will also refer as degrees of freedom. These parameters describe individual and collective movements 


\begin{tabular}{|l|c|c|c|}
\hline Quantity & Symbol & $\begin{array}{c}\text { Ring 4 } \\
\text { (ray 3) }\end{array}$ & $\begin{array}{c}\text { Ring 7 } \\
\text { (ray 2) }\end{array}$ \\
\hline \hline pitch & $p$ & $126 \mu \mathrm{m}$ & $156 \mu \mathrm{m}$ \\
\hline beam diameter & $\sigma_{L}$ & $0.25 \mathrm{~mm}$ & $0.25 \mathrm{~mm}$ \\
\hline fitting accuracy & $\Delta m_{\text {fit }}$ & $16 \mu \mathrm{m}$ & $18 \mu \mathrm{m}$ \\
\hline Distortion contrib. & $\Delta m_{\text {dis }}$ & $7 \mu \mathrm{m}$ & $7 \mu \mathrm{m}$ \\
\hline Spot accuracy & $\Delta m_{\text {spot }}$ & $17 \mu \mathrm{m}$ & $19 \mu \mathrm{m}$ \\
\hline Refraction contrib. & $\Delta m_{\text {refr }}$ & $27 \mu \mathrm{m}$ & $27 \mu \mathrm{m}$ \\
\hline $\begin{array}{l}\text { Mechanical accuracy } \\
\text { (without metrology) }\end{array}$ & $\Delta m_{m e c h}$ & $50 \mu \mathrm{m}$ & $50 \mu \mathrm{m}$ \\
\hline $\begin{array}{l}\text { Mechanical accuracy } \\
\text { (with metrology) }\end{array}$ & $\Delta m_{m e c h}$ & $20 \mu \mathrm{m}$ & $20 \mu \mathrm{m}$ \\
\hline \hline $\begin{array}{l}\text { Absolute accuracy } \\
\text { (without metrology) }\end{array}$ & $\Delta m_{\text {abs }}$ & $59 \mu \mathrm{m}$ & $60 \mu \mathrm{m}$ \\
\hline $\begin{array}{l}\text { Absolute accuracy } \\
\text { (with metrology) }\end{array}$ & $\Delta m_{\text {abs }}$ & $38 \mu \mathrm{m}$ & $39 \mu \mathrm{m}$ \\
\hline Relative accuracy & $\Delta m_{\text {rel }}$ & $17 \mu \mathrm{m}$ & $19 \mu \mathrm{m}$ \\
\hline
\end{tabular}

Table 3.3: Summary of all contributions to the overall precision with which the intersecting point of laser beams and disks can be found for the internal endcap alignment. The mechanical accuracies are estimates.

of the disks and beams. A best fit of these parameters is then made to the set of measured intersection points of the beams with the disks, by defining an error function and minimizing it with respect to the degrees of freedom. We get a set of 49 linear equations, the solution being the parameters which describe the disk and beam movements. Such a solution is not unique since, for example, any rotation of all disks around the z-axis cannot be distinguished from an (opposite) rotation of all beams about this axis. To obtain a unique solution all correlated movements have to be described and treated separately, with all their effects removed from the uncorrelated parameters (this will be described in detail in the next section). The 49 parameters split up in 27 parameters describing the movements of the disks with respect to each other, 16 parameters describing the beam movements with respect to each other and 6 parameters describing the correlated movements that the whole endcap and the complete set of beams perform with respect to each other.

\subsubsection{Layout and Parametrisation}

In the endcaps the measured laser spot position is affected by the following:

- Movement of the disks

- Deformation of the disks 
- Movement of the laser beams

- Refraction of the laser beams

- Movement of the modules with respect to the disks

- Accuracy in finding the laser spot on the module

Since there are not enough laser beams to monitor movements of each individual module, we treat the module displacements with respect to the disks as uncertainties in finding the laser spot. In the same way we consider the laser beam refraction as an additional uncertainty in determining the laser spot position. We can therefore consider the disks as rigid planes, each with 5 degrees of freedom, and the laser beams as straight lines, each with 4 degrees of freedom. Since the strip sensors, which are hit by the laser beams, measure only the $\phi$-coordinate, the degrees of freedom for the disks and the beams, which can be detected by the alignment system, are reduced to 3 and 2 respectively. These can be chosen as:

- Disk rotation around z-axis

- Disk displacements in $\mathrm{x}$ - and $\mathrm{y}$-direction

- Beam displacement in $\phi$

- Beam orientation in $\phi$

The degrees of freedom of the disks and the laser beams are not independent of each other. As mentioned above, a collective rotation of all beams around the z-axis is indistinguishible from such a rotation of all disks in the opposite direction. We therefore have to separate collective movements, which cannot be assigned unequivocally to disks or laser beams, from individual displacements which then become zero on average for all 9 disks or all 8 beams.

To give an example consider the displacement in $x$ of the endcap disks illustrated in fig.3.3. For simplicity we assume that the laser beams have kept their nominal positions, forming a ring around the nominal centers of the disks, which lie on the straight line O-L in the figure and are designated with squares. The shifted centers of the disks are designated by the crosses. The displacement of the endcap disks is decomposed into 3 parts: $\Delta x_{0}$, the collective displacement of all disks with respect to the beams, $\Delta x_{t}$, the collective shearing in $x$ of the disks with respect to the beams and $\Delta x_{k}$, the individual $\mathrm{x}$-displacement of each disk. The collective parameters $\Delta x_{0}$ and $\Delta x_{t}$ are defined in such a way that the average value and the average slope of the $\Delta x_{k}$ (and in the general case also the corresponding parametrisations of the individual beam displacements) are zero.

If this kind of separation is performed for all possible correlated movements, we end up with the following degrees of freedom:

$\Delta \phi_{k}$ : Individual disk rotation around z-axis 


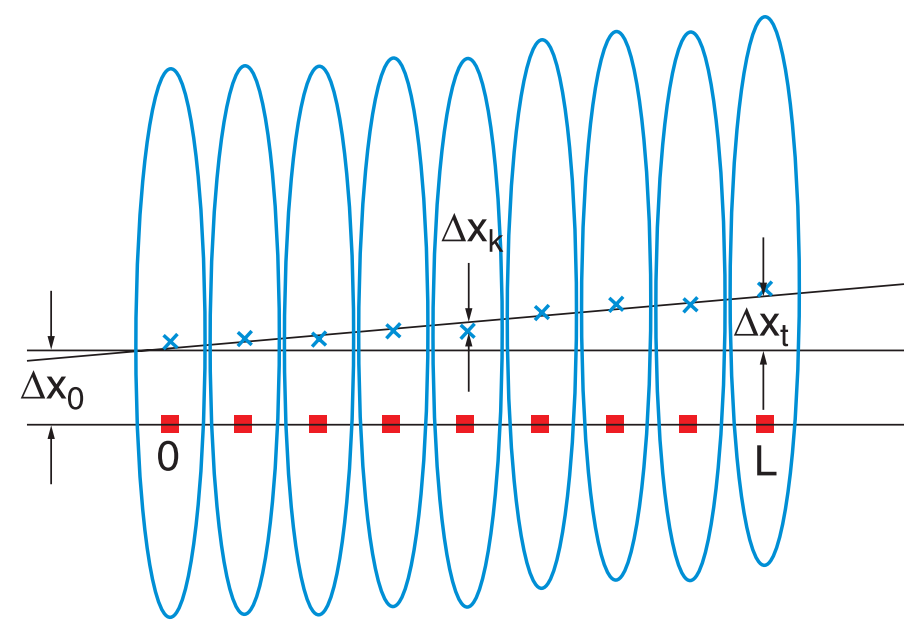

Figure 3.3: Parameters describing the disk translations along the x-axis. The sum of the individual movements $\Delta x_{k}$ is 0 as well as their average slope.

$\Delta x_{k}$ : Individual x-displacement of each disk

$\Delta y_{k}$ : Individual y-displacement of each disk

$\Delta \theta_{a i}$ : Individual beam displacement in $\phi$ on disk 1

$\Delta \theta_{b i}$ : Difference between individual beam displacements in $\phi$ on disk 1 and 9

$\Delta \phi_{0}$ : Rotation of all disks with respect to the beams

$\Delta x_{0}$ : Displacement in $\mathrm{x}$ of all disks with respect to the beams

$\Delta y_{0}$ : Displacement in y of all disks with respect to the beams

$\Delta \phi_{t}:$ Collective torsion of disks with respect to the beams

$\Delta x_{t}$ : Collective shearing in $\mathrm{x}$-direction of disks with respect to the beams

$\Delta y_{t}$ : Collective shearing in y-direction of disks with respect to the beams

Here $k$ are disk indices running from 1 to 9 , while $i$ are the beam indices ranging from 1 to 8 . The separation of the collective movements from the individual ones leads to conditions of the form (for a complete listing see appendix B):

$$
\begin{aligned}
\sum_{k} \Delta \phi_{k} & =0 \\
\sum_{k} \Delta x_{k} & =0 \\
\sum_{i} \Delta \theta_{a i} & =0
\end{aligned}
$$


It is worth noting that these conditions, which define the collective parameters, are not the only ones possible. For example, another way to do the separation of individual and collective movements would be to fix a reference system by defining the movements of the first and the last disk to be zero. The conditions used here were chosen because they seemed to be the most natural ones and because they simplify further calculations.

Since the collective degrees of freedom cannot be assigned to either disks or beams, they will create an uncertainty in the module positions. This uncertainty is not random but a systematic movement or deformation of the endcap. This means that the tracks, which are spiraling, remain spirals. The only effect is, that they may not point to the vertex any more. In principle this would not affect the pattern recognition which has to assign hits to tracks. However problems may arise if the microstrip tracker becomes misaligned with respect to the pixel detector, since the seeds for the pattern recognition algorithms are provided by the pixel sensors. There is no laser alignment system foreseen for the pixel detector, so that the task of aligning it with respect to the rest of the CMS detector has to be performed with tracks.

\subsubsection{Symmetries}

The 8-fold symmetry of the beam layout leads to some simplification of several terms:

$$
\begin{aligned}
\sum_{i} \sin \theta_{i} & =0 \\
\sum_{i} \cos \theta_{i} & =0 \\
\sum_{i} \sin ^{2} \theta_{i} & =\frac{n}{2} \\
\sum_{i} \cos ^{2} \theta_{i} & =\frac{n}{2} \\
\sum_{i} \sin \theta_{i} \cos \theta_{i} & =0
\end{aligned}
$$

Equations 3.7 and 3.8 hold for any 2-fold symmetry and equations 3.9-3.11 for any 4-fold symmetry. This simplificaton is not strictly needed to solve the system of equations, but it significantly reduces its complexity.

\subsubsection{Error function and minimization}

The next step is to describe the intersection points of disks and laser beams in terms of the parameters defined above. Assuming that all displacements are small, we can linearise the problem and thus obtain for the expected laser spot position $\Phi_{i k}$ of the i-th beam on the $\mathrm{k}$-th disk:

$$
\Phi_{i k}=\theta_{i}-\Delta \phi_{0}+\frac{\sin \theta_{i}}{R_{0}} \Delta x_{0}-\frac{\cos \theta_{i}}{R_{0}} \Delta y_{0}
$$




$$
\begin{aligned}
& -\frac{z_{k}}{L} \Delta \phi_{t}+\frac{z_{k} \sin \theta_{i}}{L \cdot R_{0}} \Delta x_{t}-\frac{z_{k} \cos \theta_{i}}{L \cdot R_{0}} \Delta y_{t} \\
& -\Delta \phi_{k}+\frac{\sin \theta_{i}}{R_{0}} \Delta x_{k}-\frac{\cos \theta_{i}}{R_{0}} \Delta y_{k} \\
& -\Delta \theta_{a i}-\frac{z_{k}}{L} \Delta \theta_{b i}
\end{aligned}
$$

with $L=z_{9}-z_{1}$ being the endcap length, and $R_{i}$ being the nominal radial positions of the laser beams.

We now define the error function $S$ as the sum of the squares of all residuals between the predicted $\left(\Phi_{i k}\right)$ and the measured $\left(Y_{i k}\right)$ values of the intersection points.

$$
S\left(\Delta \phi_{k}, \Delta x_{k}, \Delta y_{k}, \Delta \theta_{a i}, \Delta \theta_{b i}, \Delta \phi_{0}, \Delta x_{0}, \Delta y_{0}, \Delta \phi_{t}, \Delta x_{t}\right)=\sum_{i, k}\left(\Phi_{i k}-Y_{i k}\right)^{2}
$$

$S$ is now minimized with respect to all parameters. To simplify the calculation we introduce the variables $\phi_{i k}=\Phi_{i k}-\theta_{i}$ and $y_{i k}=Y_{i k}-\theta_{i}$, which describe the shift of the laser spot from its nominal position. We obtain a set of 49 linear equations, which can be solved for the degrees of freedom. The equations introduced in 3.3.1 to separate individual movements from correlated ones ensure, that there is a unique solution to this set:

$$
\begin{aligned}
\Delta \phi_{0} & =\frac{\sum_{k} z_{k}^{2} \sum_{i, k} y_{i k}-\sum_{k} z_{k} \sum_{i, k} z_{k} y_{i k}}{R_{0} n\left(\left(\sum_{k} z_{k}\right)^{2}-m \sum_{k} z_{k}^{2}\right)} \\
\Delta \phi_{t} & =\frac{L\left(m \sum_{i, k} z_{k} y_{i k}-\sum_{k} z_{k} \sum_{i, k} y_{i k}\right)}{R_{0} n\left(\left(\sum_{k} z_{k}\right)^{2}-m \sum_{k} z_{k}^{2}\right)} \\
\Delta \phi_{k}= & -\frac{z_{k} \cdot \Delta \phi_{t}}{L}-\Delta \phi_{0}-\frac{\sum_{i} y_{i k}}{R_{0} n} \\
\Delta x_{0}= & \frac{2\left(\sum_{k} z_{k} \sum_{i k} z_{k} \sin \theta_{i} y_{i k}-\sum_{k} z_{k}^{2} \sum_{i k} \sin \theta_{i} y_{i k}\right)}{n\left(\left(\sum_{k} z_{k}\right)^{2}-m \sum_{k} z_{k}^{2}\right)} \\
\Delta x_{t}= & \frac{2 L\left(\sum_{k} z_{k} \sum_{i k} \sin \theta_{i} y_{i k}-m \sum_{i k} z_{k} \sin \theta_{i} y_{i k}\right)}{n\left(\left(\sum_{k} z_{k}\right)^{2}-m \sum_{k} z_{k}^{2}\right)} \\
\Delta x_{k}= & -\frac{z_{k} \Delta x_{t}}{L}-\Delta x_{0}+\frac{2 \sum_{i} \sin \theta_{i} y_{i k}}{n} \\
\Delta y_{0}= & \frac{2\left(\sum_{k} z_{k}^{2} \sum_{i k} \cos \theta_{i} y_{i k}-\sum_{k} z_{k} \sum_{i k} z_{k} \cos \theta_{i} y_{i k}\right)}{n\left(\left(\sum_{k} z_{k}\right)^{2}-m \sum_{k} z_{k}^{2}\right)} \\
\Delta y_{t}= & \frac{2 L\left(m \sum_{i k} z_{k} \cos \theta_{i} y_{i k}-\sum_{k} z_{k} \sum_{i k} \cos \theta_{i} y_{i k}\right)}{n\left(\left(\sum_{k} z_{k}\right)^{2}-m \sum_{k} z_{k}^{2}\right)} \\
\Delta y_{k}= & -\frac{2 \sum_{i} \cos \theta_{i} y_{i k}}{n} \\
L y_{t} & \Delta y_{0}-\frac{2}{n}
\end{aligned}
$$

On the right side of these equations there appear only the measured intersection points $y_{i k}$ and known geometrical constants $\left(\theta_{i}, z_{k}, L, m\right.$ and $\left.n\right)$. Given the measured values $\Delta y_{i k}$, these equations yield the parameters which describe all the motions of the endcap. Once these parameters have been reconstructed, one can correct any 
value $\phi_{\text {meas }}$, measured by a module on the $\mathrm{k}$-th disk at radius $R$, by applying the correction for $\Delta \phi_{k}, \Delta x_{k}$ and $\Delta y_{k}$ :

$$
\phi_{\text {corr }}=\phi_{\text {meas }}+\Delta \phi_{k}-\frac{\sin \phi_{\text {meas }}}{R} \Delta x_{k}+\frac{\cos \phi_{\text {meas }}}{R} \Delta y_{k}
$$

One should not correct for the correlated displacements, unless one can make sure that they were caused by movements of the endcap and not by the laser beams. In this case an additional correction $\Delta \phi_{\text {coll }}$ can be made:

$$
\begin{aligned}
\Delta \phi_{\text {coll }}= & \Delta \phi_{0}-\frac{\sin \phi_{\text {meas }}}{R} \Delta x_{0}+\frac{\cos \phi_{\text {meas }}}{R} \Delta y_{0} \\
& +\frac{z_{k}}{L} \Delta \phi_{t}-\frac{z_{k} \sin \phi_{\text {meas }}}{L \cdot R} \Delta x_{t}+\frac{z_{k} \cos \phi_{\text {meas }}}{L \cdot R} \Delta y_{t}
\end{aligned}
$$

\subsection{Simulation of the Performance}

This section describes a simulation that was performed to assess the accuracy that can be reached with the proposed alignment system. The simulation was performed in detail for the alignment of the endcap disks with rays \#2 and \#3.

\subsubsection{Simulation Principle}

For the simulation, all degrees of freedom were generated randomly. For 9 disks, randomly generated displacements still contain a quite large coherent component i.e. the average rotation, displacements in $\mathrm{x}$ - and $\mathrm{y}$-direction, torsion and shearing. These components were calculated and subtracted from $\Delta \phi_{k}, \Delta x_{k}$ and $\Delta y_{k}$. The same was done for the correlated parts of the beam displacements. Then the $\phi$ coordinates of the intersection points of the beams with the disks were calculated. These values were smeared according to a gaussian with $\sigma=\Delta m$, the accuracy with which the intersection points are found (see sect.3.2.3, in particular table 3.3). The reconstruction procedure described in 3.3 above was then performed, yielding the 49 parameters. The difference between the generated and the reconstructed parameters is the measure for the precision of the alignment system.

\subsubsection{Results of the simulation}

It is obvious, that the accuracy of the laser alignment system is a function of the number of beams that are used, and the precision $\Delta m$ with which the intersection point of the laser beams with the disks is found. In the simulation a ring of laser beams at a given radius was used and it was assumed, that the accuracy $\Delta m$ in determining the intersection point is the same for all modules. In a first step we study the performance of the alignment system as a function of the number of beams on the ring. We then look at the dependence on $\Delta m$, the accuracy with which the intersection points are found. Finally we apply the results on the alignment system proposed for the CMS Tracker by combining two rings of laser beams with the parameters estimated in section 3.2 . 


\section{Accuracy as a function of the number of beams}

For this study a ring of laser beams at a radius of $R_{0}=564 \mathrm{~mm}$ was taken, corresponding to ray $\# 3$ of the laser alignment system (see fig.3.1). Nine disks were used at the nominal z-positions of the CMS tracker endcap. The number of beams was varied from 4 to 12 arranged perfectly symmetrically on the circumference of a circle with radius $R_{0}$. The precision with which the intersecting points are found was taken to be $50 \mu \mathrm{m}$, as estimated in section 3.2.

The residuals obtained with 8 laser beams for the parameters describing the individual disk motions and the collective movements are shown in fig.3.4 For the rotations $\phi \cdot R_{0}$ is taken, in order to compare it with the translations. The parameter that is reconstructed best is $\phi_{0} \cdot R_{0}$, since all the beam spots are sensitive to a collective rotation of the disks with respect to the beams. The residual distributions of the global translations $\Delta x_{0}$ and $\Delta y_{0}$ are wider by a factor of $\sqrt{2}$, since only half of the beam spots effectively sense each of these displacements. The same ratio of $\sqrt{2}$ appears between the individual disk rotation $\left(\Delta \phi_{k}\right)$ and translation $\left(\Delta x_{k}\right.$ or $\left.\Delta y_{k}\right)$, and between torsion $\left(\Delta \phi_{t}\right)$ and shearing of the endcap $\left(\Delta x_{t}\right.$ or $\left.\Delta y_{t}\right)$.

Figure 3.5 shows the r.m.s. width of the distributions as a function of the number of beams. The symbols are the results from the simulation, while the curves show the analytical computation as summarized in appendix B. As can be seen, using 8 beams, all parameters are reconstructed with an accuracy better than $30 \mu \mathrm{m}$. Note that the simplification introduced in 3.3.2 for the 8-fold symmetry does not introduce a significant deterioration of the residuals in the cases where this symmetry is not given (e.g. the results for an odd number of beams do not differ from the analytical curve).

\section{Accuracy as function of $\Delta m$}

For this study we take 8 beams at a radius of $564 \mathrm{~mm}$ as foreseen for ray \#3. Figure 3.6 shows the results with $\Delta m$ ranging from $20 \mu \mathrm{m}$ to $100 \mu \mathrm{m}$. Again the symbols are the results from the simulation, while the lines are the analytical calculation. As one expects, the width of the residual distributions is directly proportional to the measuring precision $\Delta m$. If we take as a worst case for ray $\# 3$ the absolute accuracy without metrology as $60 \mu \mathrm{m}$ (see 3.2.3), we can see that all parameters are reconstructed with a precision better than $35 \mu \mathrm{m}$.

\section{Combining rays 2 and 3}

The results presented so far were for ray \#3, passing through the modules of ring 4 located at $R_{0}=564 \mathrm{~mm}$. The proposed alignment system foresees one more set of beams to monitor the internal alignment of the endcaps. This set constitutes ray \#2 traversing the modules of ring 7 at a radius $R_{0}=1045 \mathrm{~mm}$. The results presented above do not depend on $R_{0}$, since all angles $\phi$ which appear in the equations are multiplied with $R_{0}$. We have seen, that the r.m.s. width of the residuals is directly proportional to $\Delta m$. Thus using the estimates for $\Delta m$ in table 3.3 , we can 


\begin{tabular}{|c|c|c|c|c|c|c|c|}
\hline $\begin{array}{c}\text { absolute } \\
\text { (no metrology) }\end{array}$ & $\Delta m$ & $\sigma_{\phi_{k}}$ & $\sigma_{x_{k} / y_{k}}$ & $\sigma_{\phi_{0}}$ & $\sigma_{x_{0} / y_{0}}$ & $\sigma_{\phi_{t}}$ & $\sigma_{x_{t} / y_{t}}$ \\
\hline \hline ray 3 & 59 & 18 & 26 & 12 & 17 & 21 & 30 \\
\hline ray 2 & 60 & 19 & 26 & 12 & 17 & 22 & 31 \\
\hline combined & & 13 & 18 & 8 & 12 & 15 & 22 \\
\hline \hline $\begin{array}{c}\text { absolute } \\
\text { (with metrology) }\end{array}$ & $\Delta m$ & $\sigma_{\phi_{k}}$ & $\sigma_{x_{k} / y_{k}}$ & $\sigma_{\phi_{0}}$ & $\sigma_{x_{0} / y_{0}}$ & $\sigma_{\phi_{t}}$ & $\sigma_{x_{t} / y_{t}}$ \\
\hline \hline ray 3 & 38 & 12 & 17 & 8 & 11 & 14 & 20 \\
\hline ray 2 & 39 & 12 & 17 & 8 & 11 & 14 & 20 \\
\hline \hline combined & & 8 & 12 & 6 & 8 & 10 & 14 \\
\hline \hline relative & $\Delta m$ & $\sigma_{\phi_{k}}$ & $\sigma_{x_{k} / y_{k}}$ & $\sigma_{\phi_{0}}$ & $\sigma_{x_{0} / y_{0}}$ & $\sigma_{\phi_{t}}$ & $\sigma_{x_{t} / y_{t}}$ \\
\hline ray 3 & 32 & 10 & 14 & 7 & 9 & 12 & 16 \\
\hline ray 2 & 33 & 10 & 15 & 7 & 10 & 12 & 17 \\
\hline combined & & 7 & 10 & 5 & 7 & 8 & 12 \\
\hline \hline
\end{tabular}

Table 3.4: Calculated precisions for the reconstruction of the parameters for the different scenarios. All values are in $\mu \mathrm{m}$

calculate the resulting precision for both sets of rays in the different scenarios (relative measurement, absolute measurement with and without metrology). The results are summarized in table 3.4 for all parameters. They show, that for all scenarios the parameters can be reconstructed better than the $100 \mu \mathrm{m}$ required for pattern recognition to work reliably.

The performance limit of the alignment system is given by the uncertainty of the module position on the disk, which is irreducible. For all the modules, which are not hit by the laser beams, one can correct for the movements and deformations of the mechanical structure. Any displacement of those modules with respect to the disks cannot be assessed. For this reason, it makes no sense to monitor the disk displacements with a much better precision than the mechanical accuracy which is around $20 \mu \mathrm{m}$ or $50 \mu \mathrm{m}$ depending on whether a metrology has been performed or not. 

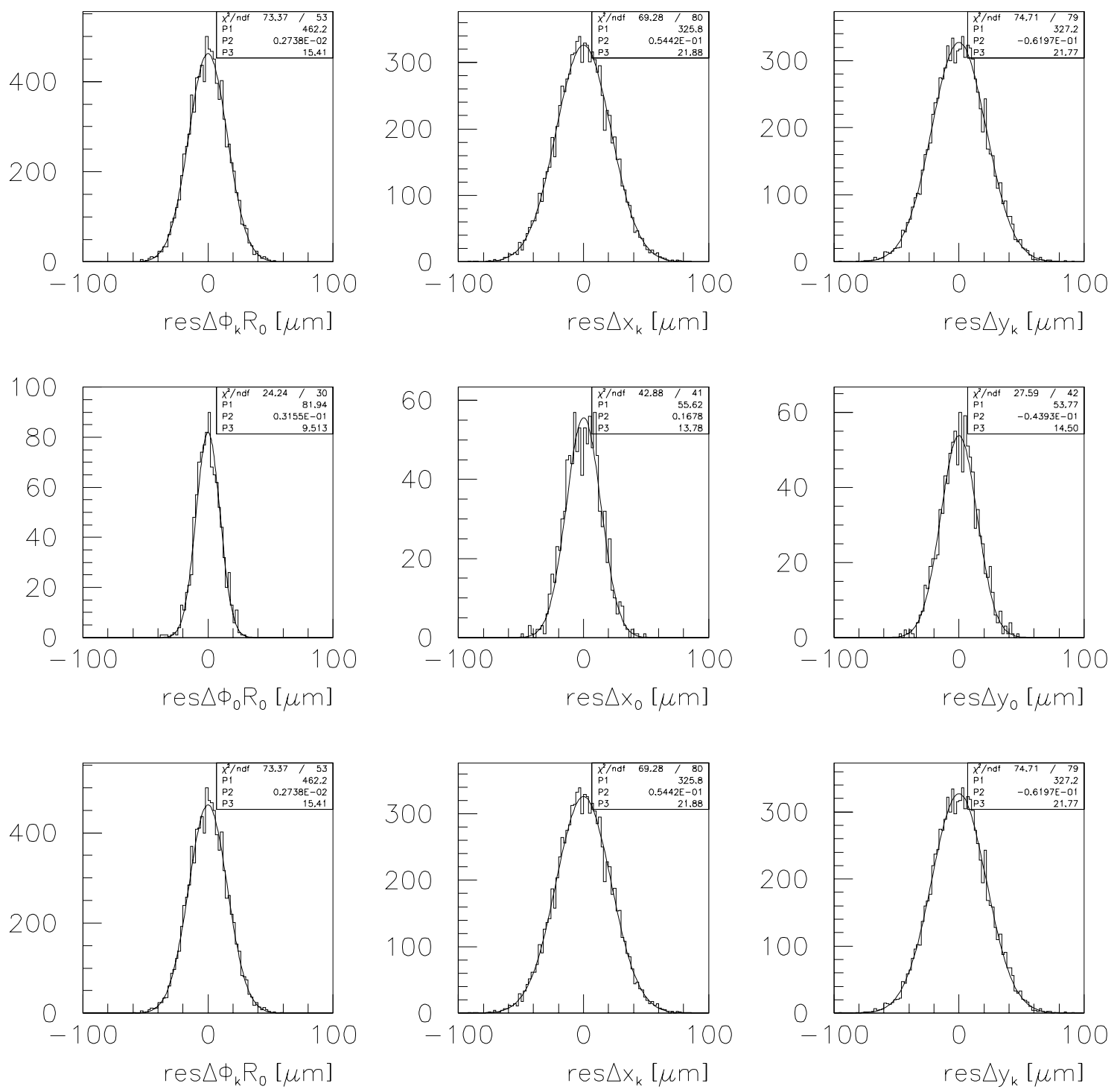

Figure 3.4: Distributions of the residuals for the individual disk displacements and for the collective movements. A ring of 8 beams at radius $R_{0}=564 \mathrm{~mm}$ was used. 


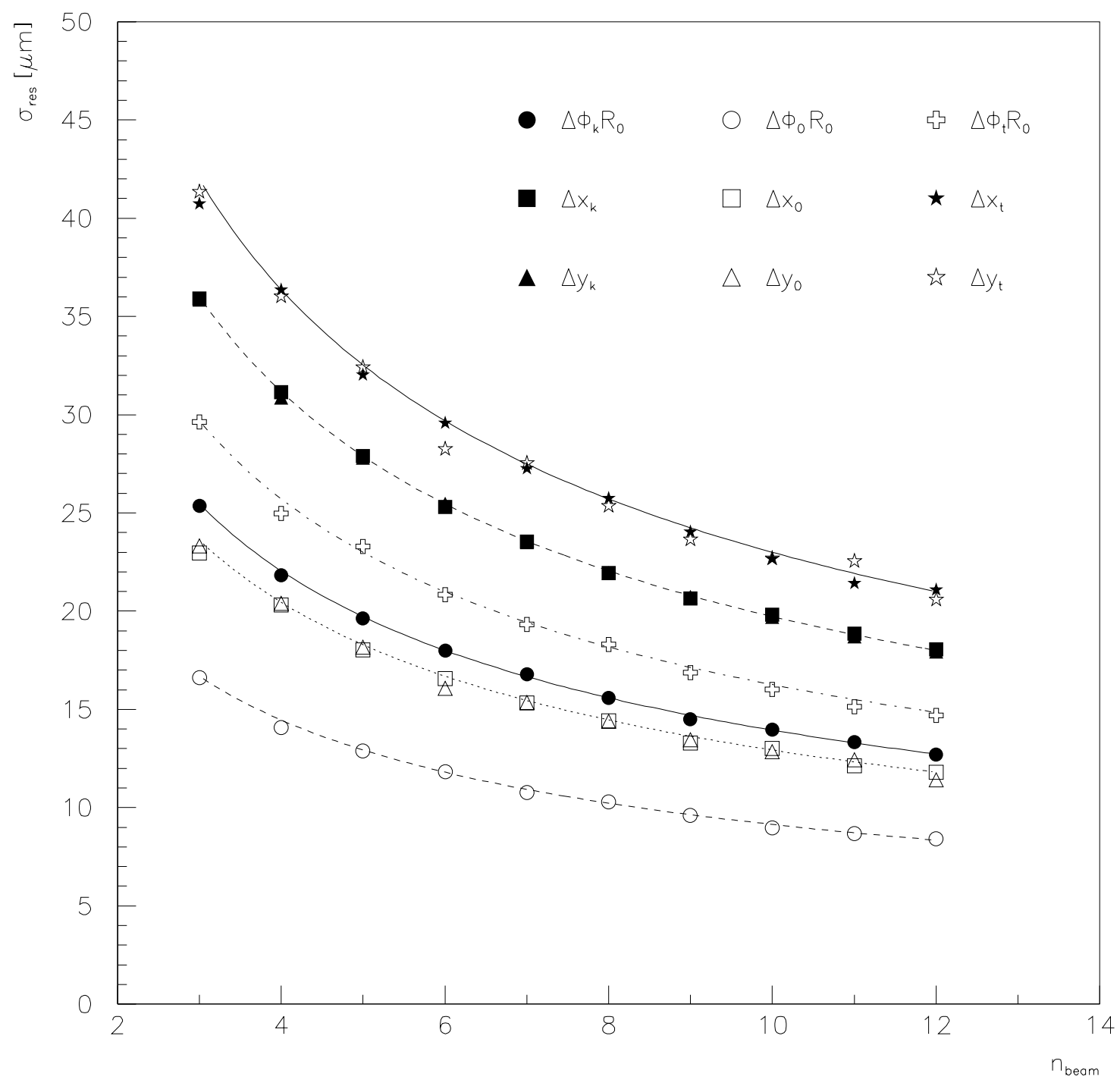

Figure 3.5: Precision of the reconstructed parameters as a function of the number of beams. The beams are at a radius $R_{0}=564 \mathrm{~mm}$ and are equally spaced in $\phi$. 


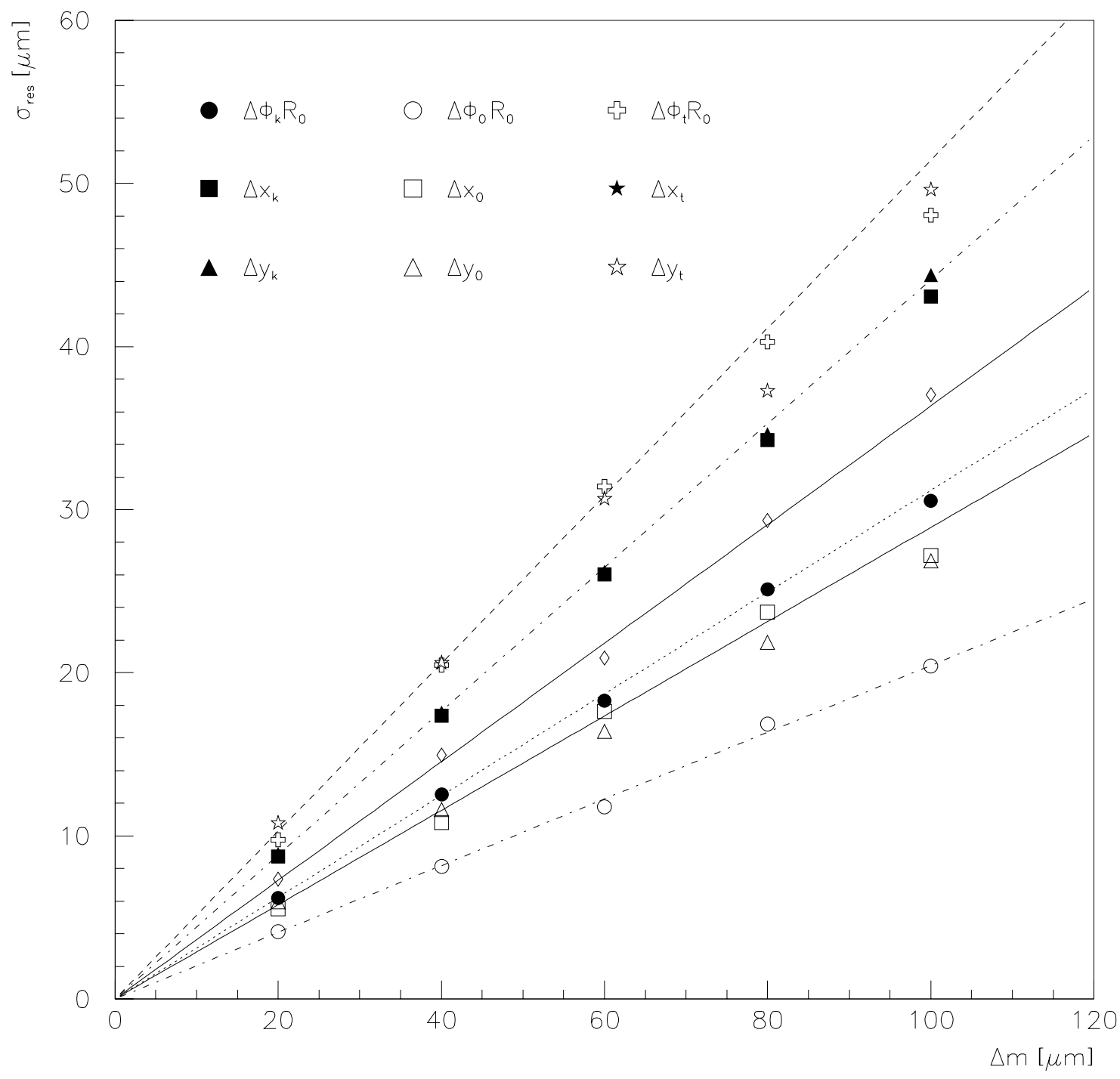

Figure 3.6: Precision of the reconstructed parameters as a function of the accuracy $\Delta m$ with which the intersection point of laser beams and disks is found. There are 8 beams which are equally spaced in $\phi$. The results do not depend on $R_{0}$. 


\section{Chapter 4}

\section{Conclusion}

\subsection{Summary}

In this work we presented a laser alignment system for the CMS silicon microstrip detector. The purpose of the system is to monitor movements of the mechanical structure of the tracker with a precision better than $100 \mu \mathrm{m}$. An alignment of the sensors with this precison is needed to ensure that the online track finding algorithms work reliably. The principle of the laser alignment system is to foresee infrared laser beams in the tracker, which are detected by the microstrip sensors. Since silicon is partially transparent for infrared light these laser beams may cross several layers of sensors. Movements of the mechanical structures can then be inferred from motions of the laser spots on the modules.

In chapter 2 we described how to remove the backplane metalization from the sensor zone where the laser beams pass through the modules. We then studied the transparency and the reflectance of the sensors and found an overall transmission around 20\% for wavelengths between 1060 and $1080 \mathrm{~nm}$. Interference effects were observed coming from reflections at the two silicon-air interfaces of a sensor. Due to these interference effects the signal response to the laser light of different strips can vary up to a factor of 3 , causing a distortion of the beam profile that is detected. The interfernce patterns that were measured allowed us also to reconstruct the changes of the sensor thickness over the area of one sensor. They are in the order of a few $\mu \mathrm{m}$ on detectors with $50 \times 60 \mathrm{~mm}^{2}$ size. With this result we were able to estimate the refraction that a beam suffers when passing through a silicon sensor. It was found that kinks in the beams up to $0.3 \mathrm{mrad}$ can occur.

Another important issue is the precision $\Delta m_{\text {spot }}$ with which the laser spot can be found on the module. In section 2.5 we saw that it is a function of the laser beam width $\sigma_{L}$, the signal-to-noise $(\mathrm{S} / \mathrm{N})$-ratio and the strip pitch. The effective $\mathrm{S} / \mathrm{N}$-ratio can be enhanced by averaging over a few hundred laser pulses, so that it becomes possible to process signals from sensors with a $\mathrm{S} / \mathrm{N}$-ratio $<1$ for a single pulse. $\Delta m$ is also influenced by the distortions of the beam profile introduced by the interference. For typical microstrip modules (pitch 50-200 $\mu \mathrm{m}$, S/N-ratio 10-100) and $\sigma_{L} \approx 0.25-1 \mathrm{~mm}$, the overal spot accuracy $\Delta m$ lies in the range $10-50 \mu \mathrm{m}$. 
In chapter 3 the alignment system which has been proposed for the CMS microstrip tracker was presented. The laser sources are located outside the CMS detector and the light is brought into the tracker with optical fibers. The laser beams are generated by collimators sitting on the end of the fibers and small prisms which send out two back-to-back beams. The internal alignment of the endcap disks is achieved by two sets of 8 beams in each endcap. These beams are arranged in rings running parallel to the z-axis. The alignment of the four subdetectors (inner barrel, outer barrel and the two endcaps) with respect to each other is done with another set of 8 laser beams. These beams run parallel to $\mathrm{z}$ in between inner and outer barrel. Beam splitters reflect part of the light onto the silicon modules in the barrel subdetectors. The beams also penetrate both endcaps generating a signal on 4 silicon layers. Finally two sets of 6 beams are foreseen for the alignment of the whole tracker with respect to the CMS muon system. These beams come from outside the tracker and are routed with periscopes into the endcaps where they are detected by the silicon sensors.

To study the alignment precision that can be reached, the beams performing the internal endcap alignment were chosen. Section 3.3 describes how the motions of the mechanical structure of the endcap can be assesed from the measured laser beam spot positions. To this purpose a parametrization of possible movements of the mechanical structure of the endcaps is described. In this parametrization care is taken to separate collective disk and beam movements from the individual displacements which are zero in their average. For one endcap and 8 laser beams a total of 49 parameters describes all these movements. A linear fit of the parameters to the set of measured beam positions is performed The separation of collective and individual movements ensures that there is a unique solution to this linear regression.

In section 3.4 a simulation of the alignment performance in the endcaps is presented. Random displacements of the endcap disks were performed and the resulting intersection points of the laser beams and the disks were calculated. A gaussian smearing was applied to these values to account for the error which is made when measuring the intersection points. From this set of simulated data the values of the parameters were reconstructed and compared to the generated ones. It turns out, that all the parameters can be reconstructed with a precision better than $40 \mu \mathrm{m}$. This is even the case when less than 8 beams are in one ring, showing that there is redundancy in the system for the case that some laser beam fails. The precisions obtained from the simulation agree very well with the ones obtained from an analytical calculation.

\subsection{Open Issues}

There are a few open issues concerning the laser alignment system for the CMS tracker which could not be adressed in this work and which are worth noting.

The most important is certainly the radiation hardness of the alignment system. This concerns the optical components inside the tracker i.e. the fibers, collimators 
and prisms. Studies have shown, that the materials used are quite radiation hard, but tests on prototypes of the finally used components are still to be done. Radiation hardness is also an issue for the sensors that are illuminated by the laser beams. Radiation tests have to be performed to make sure that there is no additional deterioration of the sensors in the region where the backplane metalization was removed, and that the transparency of the silicon and the dielectric layer does not suffer from the irradiation.

Another step which has to be taken is the construction of a full scale prototype to check whether the accuracies which were estimated can be reached in a real system.

Filnally more work has to be done for the integration into the CMS DAQ and software. 


\section{Acknowledgements}

First of all I would like to thank Prof. K. Lübelsmeyer and Prof. St. Schael for giving me the opportunity to attain a doctorate at the I. Physikalisches Institut, RWTH Aachen and for their support througout this work.

I would also like to thank R. Siedling and the entire staff of the mechanical and electronics workshops of the institute for their help in builduing up the setups which were needed.

I am especially indebted to Dr. D. Pandoulas who kept encouraging me over the past years and gave valuable input to this work through many discussions. He also read the manuscript, making helpful suggestions and improving the english.

Many thanks also to St. König, A. Ostapchouk and F. Raupach for reading the manuscript and giving their comments.

Last but not least I would like to thank all my colleagues at the institute who turned the everyday labour into a nice time worth remembering. 


\section{Appendix A}

\section{Accuracy in determining the laser spot on the module}

In the following, a short derivation for the accuracy in determining the laser spot on the module will be given. This calculation will be done for a perfect gaussian profile. $x_{i}$ is the position of a strip and $y_{i}$ the signal in this strip. $N$ is the number of strips which are used to find the position and we will assume that the strips are arranged symetrically around the mean value $m$. As first approximation we will take the position $m$ of the profile as the center of gravity of the strip signals.

$$
m=\frac{\sum x_{i} y_{i}}{\sum y_{i}}
$$

For the error $\Delta m$ we get:

$$
\Delta m^{2}=\sum_{k}\left|\frac{\partial m}{\partial y_{k}}\right|^{2} \Delta y^{2}
$$

where the noise $\Delta y$ shall be the same for all strips.

$$
\begin{gathered}
\Delta m^{2}=\Delta y^{2} \sum_{k}\left(\frac{m-x_{k}}{\sum y_{i}}\right)^{2} \\
\Delta m^{2}=\left(\frac{\Delta y}{\sum y_{i}}\right)^{2} \cdot\left(N m^{2}-2 m \sum x_{k}+\sum x_{k}^{2}\right) \\
\Delta m=\frac{\Delta y}{\sum y_{i}} \cdot \sqrt{N m^{2}-2 m \sum x_{k}+\sum x_{k}^{2}}
\end{gathered}
$$

For a gaussian profile with width $\sigma_{L}$ and the height $y_{0}$, we have for the area under the curve:

$$
\sqrt{2 \pi} \cdot \sigma_{L} \cdot y_{0} \approx \sum y_{i} \cdot p
$$

the pitch $p$ being the distance between two strips.

$$
\Delta m=\frac{\Delta y}{y_{0}} \cdot \frac{1}{\sigma_{L}} \cdot p \cdot \frac{1}{\sqrt{2 \pi}} \cdot \sqrt{N m^{2}-2 m \sum x_{k}+\sum x_{k}^{2}}
$$


If the strips that are used for the COG calculation are arranged symetrically around $m$ we can write $^{1}$ :

$$
\begin{aligned}
\sum x_{k} & =N \cdot m \\
\sum x_{k}^{2} & =N \cdot m^{2}+\frac{p^{2}}{12} \cdot\left(N^{3}-N\right) \\
& \approx N \cdot m^{2}+\frac{p^{2}}{12} \cdot N^{3}
\end{aligned}
$$

Inserting in A.7:

$$
\Delta m=\frac{\Delta y}{y_{0}} \cdot \frac{1}{\sigma_{L}} \cdot p^{2} \cdot \frac{1}{\sqrt{12} \sqrt{2 \pi}} \cdot \sqrt{N^{3}}
$$

$y_{0} / \Delta y$ is the definition we have been using for the $\mathrm{S} / \mathrm{N}$-ratio of the beam profile. The number of points $N$ used for the calculation should be a function of the laser width $\sigma_{L}$ and the pitch $p$. For the COG algorithm best results are obtained, if the range is $4 \cdot \sigma_{L}$, so that we write:

$$
N=4 \cdot \frac{\sigma_{L}}{p}
$$

Inserting into A.11, we get:

$$
\begin{aligned}
& \Delta m=\left(\frac{S}{N}\right)^{-1} \cdot \sqrt{\frac{8}{3 \pi}} \cdot \sqrt{p \cdot \sigma_{L}} \\
& \Delta m \propto\left(\frac{S}{N}\right)^{-1} \cdot \sqrt{p \cdot \sigma_{L}}
\end{aligned}
$$

\footnotetext{
${ }^{1}$ Note that this is an idealization, since in the general case the center $m$ doesn't coincide with the center of a strip (for odd $N$ ) or the middle in between two strips (for even $N$ ). In practice it can even be harder to match $m$ with the center of the strip range, if the signals are noisy and the laser width $\sigma_{L}$ is large. This uncertainty can be minimized by using an iterative approach where $m$ is calculated repeatedly and the strip range is adapted in every step.
} 


\section{Appendix B}

\section{Equations used for the reconstruction}

This appendix summarizes the equations used to reconstruct the deformations of the endcap with help of the laser alignment system.

\section{Variables describing the ideal geometry}

The endcap geometry and one set of beams are described by the following variables:

n: Number of beams.

$R_{0}$ : Nominal radius of beams.

$\theta_{i}$ : Nominal $\phi$-position of $i$-th beam.

$m$ : Number of disks.

$z_{k}:$ Z-position of $k$-th disk.

$L$ : Length of endcap $\left(z_{m}-z_{1}\right)$.

The index $i$ is used to enumerate the beams, while $k$ is used to enumerate disks.

\section{The parameters describing the movements of the endcap}

$\Delta \phi_{k}$ : Individual disk rotation around z-axis.

$\Delta x_{k}$ : Individual x-displacement of each disk.

$\Delta y_{k}$ : Individual y-displacement of each disk.

$\Delta \theta_{a i}$ : Individual beam displacement in $\phi$ on disk 1.

$\Delta \theta_{b i}$ : Individual beam displacement in $\phi$ between disk 1 and disk 9 . 
$\Delta \phi_{0}$ : Rotation of all disks with respect to the beams.

$\Delta x_{0}$ : Displacement in $\mathrm{x}$ of all disks with respect to the beams.

$\Delta y_{0}$ : Displacement in $\mathrm{y}$ of all disks with respect to the beams.

$\Delta \phi_{t}$ : Collective torsion of disks with respect to the beams.

$\Delta x_{t}$ : Collective shearing in $\mathrm{x}$-direction of disks with respect to the beams.

$\Delta y_{t}$ : Collective shearing in y-direction of disks with respect to the beams.

Here $k$ are disk indices running from 1 to 9 , while $i$ are the beam indices ranging from 1 to 8 . We therefore have a total of 49 parameters.

\section{Separation of correlated parameters}

To separate the collective movements from the individual ones, we require:

$$
\begin{aligned}
\sum_{k} \Delta \phi_{k} & =0 \\
\sum_{k} \Delta x_{k} & =0 \\
\sum_{k} \Delta y_{k} & =0 \\
\sum_{i} \Delta \theta_{a i} & =0 \\
\sum_{i} \Delta \theta_{b i} & =0 \\
\sum_{i} \sin \theta_{i} \Delta \theta_{a i} & =0 \\
\sum_{i} \sin \theta_{i} \Delta \theta_{b i} & =0 \\
\sum_{i} \cos \theta_{i} \Delta \theta_{a i} & =0 \\
\sum_{i} \cos \theta_{i} \Delta \theta_{b i} & =0 \\
\sum_{k} z_{k} \Delta \phi_{k} & =0 \\
\sum_{k} z_{k} \Delta x_{k} & =0 \\
\sum_{k} z_{k} \Delta y_{k} & =0
\end{aligned}
$$

Here $\theta_{i}$ means the nominal $\phi$-angle of the beams and $z_{k}$ are the nominal z-positions of the disks. 


\section{Symmetry considerations}

The 8-fold symmetry of the beam layout leads to some simplification of several terms.

$$
\begin{aligned}
\sum_{i} \sin \theta_{i} & =0 \\
\sum_{i} \cos \theta_{i} & =0 \\
\sum_{i} \sin ^{2} \theta_{i} & =\frac{n}{2} \\
\sum_{i} \cos ^{2} \theta_{i} & =\frac{n}{2} \\
\sum_{i} \sin \theta_{i} \cos \theta_{i} & =0
\end{aligned}
$$

Equations B.1 and B.2 hold for any 2-fold symmetry and the remaining three for any 4-fold symmetry.

\section{Linearised intersection points}

Assuming that all displacements are small, we can linearise the problem and obtain for the laser spot position $\phi_{i k}$ as function of the parameters:

$$
\begin{aligned}
\phi_{i k}= & \theta_{i}-\Delta \phi_{0}+\frac{\sin \theta_{i}}{R_{0}} \Delta x_{0}-\frac{\cos \theta_{i}}{R_{0}} \Delta y_{0} \\
& -\frac{z_{k}}{L} \Delta \phi_{t}+\frac{z_{k} \sin \theta_{i}}{L \cdot R_{0}} \Delta x_{t}-\frac{z_{k} \cos \theta_{i}}{L \cdot R_{0}} \Delta y_{t} \\
& -\Delta \phi_{k}+\frac{\sin \theta_{i}}{R_{0}} \Delta x_{k}-\frac{\cos \theta_{i}}{R_{0}} \Delta y_{k} \\
& -\left(\frac{z_{k}}{L}-1\right) \Delta \theta_{a i}-\frac{z_{k}}{L} \Delta \theta_{b i}
\end{aligned}
$$

with $L=z_{9}-z_{1}$ being the endcap length, and $R_{0}$ being the nominal radius of the laser beams.

\section{Error function and minimazation}

The error function $S$ is given as sum over the squares of the differences between calculated intersection points $\phi_{i k}$ and the measured intersection points $y_{i k}$.

$$
S=\sum_{i, k}\left(\phi_{i k}-y_{i k}\right)^{2}
$$

The error function is minimized with respect to all parameters. We obtain a set of 49 linear equations, which can be solved for the degrees of freedom.

$$
m \cdot n \cdot \Delta \phi_{0}+n \sum_{k} \frac{z_{k}}{L} \Delta \phi_{t}=-\sum_{i k} y_{i k}
$$




$$
\begin{aligned}
\frac{m \cdot n}{2 R_{0}} \Delta x_{0}+\sum_{k} z_{k} \frac{n}{2 L R_{0}} \Delta x_{t} & =\sum_{i k} \sin \theta_{i} y_{i k} \\
\frac{m \cdot n}{2 R_{0}} \Delta y_{0}+\sum_{k} z_{k} \frac{n}{2 L R_{0}} \Delta y_{t} & =-\sum_{i k} \cos \theta_{i} y_{i k} \\
n \sum_{k} z_{k} \Delta \phi_{0}+\frac{n}{L} \sum_{k} z_{k}^{2} \Delta \phi_{t} & =-\sum_{i k} z_{k} y_{i k} \\
\frac{n}{2 R_{0}} \sum_{k} z_{k} \Delta x_{0}+\frac{n}{2 L \cdot R_{0}} \sum_{k} z_{k}^{2} \Delta x_{t} & =\sum_{i k} z_{k} \sin \theta_{i} y_{i k} \\
\frac{n}{2 R_{0}} \sum_{k} z_{k} \Delta y_{0}+\frac{n}{2 L \cdot R_{0}} \sum_{k} z_{k}^{2} \Delta y_{t} & =-\sum_{i k} z_{k} \cos \theta_{i} y_{i k} \\
n \cdot \Delta \phi_{0}+\frac{n \cdot z_{k}}{L} \Delta \phi_{t}+n \cdot \Delta \phi_{k} & =-\sum_{i} y_{i k} \\
\frac{n}{2 R_{0}} \Delta x_{0}+\frac{n}{2 L \cdot R_{0}} z_{k} \Delta x_{t}+\frac{n}{2 R_{0}} \Delta x_{k} & =\sum_{i} \sin \theta_{i} y_{i k} \\
\frac{n}{2 R_{0}} \Delta y_{0}+\frac{n}{2 L \cdot R_{0}} z_{k} \Delta y_{t}+\frac{n}{2 R_{0}} \Delta y_{k} & =-\sum_{i} \cos \theta_{i} y_{i k}
\end{aligned}
$$

\section{Solutions of the equations}

$$
\begin{aligned}
\Delta \phi_{0} & =\frac{\sum_{k} z_{k}^{2} \sum_{i, k} y_{i, k}-\sum_{k} z_{k} \sum_{i, k} z_{k} y_{i, k}}{R_{0} n\left(\left(\sum_{k} z_{k}\right)^{2}-m \sum_{k} z_{k}^{2}\right)} \\
\Delta \phi_{t} & =\frac{L\left(m \sum_{i, k} z_{k} y_{i, k}-\sum_{k} z_{k} \sum_{i, k} y_{i, k}\right)}{R_{0} n\left(\left(\sum_{k} z_{k}\right)^{2}-m \sum_{k} z_{k}^{2}\right)} \\
\Delta \phi_{k} & =-\frac{z_{k} \cdot \Delta \phi_{t}}{L}-\Delta \phi_{0}-\frac{\sum_{i} y_{i, k}}{R_{0} n} \\
\Delta x_{0}= & \frac{2\left(\sum_{k} z_{k} \sum_{i k} z_{k} \sin \theta_{i} y_{i k}-\sum_{k} z_{k}^{2} \sum_{i k} \sin \theta_{i} y_{i k}\right)}{n\left(\left(\sum_{k} z_{k}\right)^{2}-m \sum_{k} z_{k}^{2}\right)} \\
\Delta x_{t}= & \frac{2 L\left(\sum_{k} z_{k} \sum_{i k} \sin \theta_{i} y_{i k}-m \sum_{i k} z_{k} \sin \theta_{i} y_{i k}\right)}{n\left(\left(\sum_{k} z_{k}\right)^{2}-m \sum_{k} z_{k}^{2}\right)} \\
\Delta x_{k}= & -\frac{z_{k} \Delta x_{t}}{L}-\Delta x_{0}+\frac{2 \sum_{i} \sin \theta_{i} y_{i k}}{n} \\
\Delta y_{0}= & \frac{2\left(\sum_{k} z_{k}^{2} \sum_{i k} \cos \theta_{i} y_{i k}-\sum_{k} z_{k} \sum_{i k} z_{k} \cos \theta_{i} y_{i k}\right)}{n\left(\left(\sum_{k} z_{k}\right)^{2}-m \sum_{k} z_{k}^{2}\right)} \\
\Delta y_{t}= & \frac{2 L\left(m \sum_{i k} z_{k} \cos \theta_{i} y_{i k}-\sum_{k} z_{k} \sum_{i k} \cos \theta_{i} y_{i k}\right)}{n\left(\left(\sum_{k} z_{k}\right)^{2}-m \sum_{k} z_{k}^{2}\right)} \\
\Delta y_{k}= & -\frac{2 z_{k} \Delta y_{t}}{L}-\Delta y_{0}-\frac{2 \sum_{i} \cos \theta_{i} y_{i k}}{n}
\end{aligned}
$$

\section{Errors for the reconstructed parameters}

The errors $\sigma_{\text {par }}$ of the reconstructed parameters are a function of the number of beams $n$ and the accuracy $\Delta m$ with which the intersection points of beams and 
disks are measured. Furthermore they depend on the geometry desscribed by $R_{0}, z_{k}$ and $m$. The errors are obtained by performing error propagation on the equations from the preceeding section.

$$
\begin{aligned}
\sigma_{\phi_{0}} & =\frac{\Delta m}{R_{0} \sqrt{n}} \sqrt{\frac{\sum_{k} z_{k}^{2}}{m \sum_{k} z_{k}^{2}-\left(\sum_{k} z_{k}\right)^{2}}} \\
\sigma_{x_{0} / y_{0}} & =\frac{\Delta m}{\sqrt{n}} \sqrt{\frac{2 \cdot \sum_{k} z_{k}^{2}}{m \sum_{k} z_{k}^{2}-\left(\sum_{k} z_{k}\right)^{2}}} \\
\sigma_{\phi_{t}} & =\frac{L \Delta m}{R_{0} \sqrt{n}} \sqrt{\frac{m}{m \sum_{k} z_{k}^{2}-\left(\sum_{k} z_{k}\right)^{2}}} \\
\sigma_{x_{t} / y_{t}} & =\frac{L \Delta m}{\sqrt{n}} \sqrt{\frac{2 m}{m \sum_{k} z_{k}^{2}-\left(\sum_{k} z_{k}\right)^{2}}} \\
\sigma_{\phi_{j}} & =\frac{\Delta m}{R_{0} \sqrt{n}} \sqrt{1-\frac{\sum_{k} z_{k}^{2}+m z_{j}^{2}-2 z_{j} \sum_{k} z_{k}}{m \sum_{k} z_{k}^{2}-\left(\sum_{k} z_{k}\right)^{2}}} \\
\sigma_{x_{j} / y_{j}} & =\frac{\Delta m}{R_{0} \sqrt{n}} \sqrt{2-\frac{2\left(\sum_{k} z_{k}^{2}+m z_{j}^{2}-2 z_{j} \sum_{k} z_{k}\right)}{m \sum_{k} z_{k}^{2}-\left(\sum_{k} z_{k}\right)^{2}}}
\end{aligned}
$$




\section{Bibliography}

[1] http://lephiggs.web.cern.ch/LEPHIGGS/www/Welcome.html.

[2] Search for the Standard Model Higgs Boson at LEP. LHWG-Note-2001-03 (hep-ex/0107029).

[3] The Large Hadron Collider: Conceptual design. CERN-AC-95-05-LHC.

[4] Michael Spira. Higgs boson production and decay at future machines. 1997.

[5] M. Grazzini. Higgs boson production at Tevatron run II and LHC. 2001.

[6] Michael Spira. Higgs boson production at the LHC. 1997.

[7] J. F. Gunion, H. E. Haber, G. Kane, and S. Dawson. The Higgs Hunter's Guide. Addison Wesley, 1990.

[8] CERN/LHCC 94-38. The Compact Muon Solenoid, Technical Proposal. Technical report, CERN, 1994.

[9] G. Acquistapace et al. CMS, the magnet project: Technical design report. CERN-LHCC-97-10.

[10] CMS, the Compact Muon Solenoid. Muon technical design report. CERNLHCC-97-32.

[11] CMS: The hadron calorimeter project. Technical design report. CERN-LHCC97-31.

[12] CMS: The electromagnetic calorimeter. Technical design report. CERN-LHCC97-33.

[13] CMS: The tracker project. Technical design report. CERN-LHCC-98-6.

[14] Addendum to the CMS tracker TDR. CERN-LHCC-2000-016.

[15] D. Abbaneo. The tracker layout working page, http://duccio.home.cern.ch/duccio/layout/lay.html. 
[16] Technical Specification: Supply of Silicon Micro-Strip Sensors for The CMS Silicon Strip Tracker (SST). Invitation to Tender: IT-2777/EP/CMS, October 2000.

[17] K. Gill et al. Radiation damage by neutrons and photons to silicon detectors. Nucl. Instrum. Meth., A322:177-188, 1992.

[18] M. Moll, H. Feick, E. Fretwurst, and G. Lindstrom. Correlation between a deep hole trap and the reverse annealing effect in neutron-irradiated silicon detectors. Nucl. Instrum. Meth., A409:194-197, 1998.

[19] S. Dasu et al. CMS. The TriDAS project. Technical design report, vol. 1: The trigger systems. CERN-LHCC-2000-038.

[20] A. Bondar et al. Influence of misalignment on CMS central tracker performance. CMS Internal Note CMS IN 1997/016, CERN, 1997.

[21] J. Vandenhirtz, W. Wallraff, and M. Weisgerber. Space flight experience with the AMS infrared tracker alignment system. In Proceedings of the 27th International Cosmic Ray Conference (ICRC), 2001.

[22] J. Vandenhirtz. Ein Infrarot Laser Positions-Kontroll-System für das AMS Experiment. PhD thesis, RWTH Aachen, 2001.

[23] Jr. G.E. Jellison and D.H. Lowndes. Optical absorption coefficient of silicon at $1.152 \mu$ at elevated temperatures. Appl. Phys. Lett., 41(7):594-596, 1982.

[24] St. König. Aufbau eines Teststandes für Silizium-Zähler des Spurdetektors im CMS-Experiment. Diploma thesis at RWTH Aachen, 1999.

[25] A. Ostapchouk et al. The Alignment System of the CMS Tracker. CMS Note 2001/053, CERN, 2001.

[26] M. Fernández García. An Alignment System for the CMS Experiment at the Large Hadron Collider. PhD thesis, CSIC Universidad de Cantabria, October 2001. 


\section{List of Figures}

1.1 Log-likelihood ratio as function of the Higgs mass [2]. The shaded regions denominate the $1 \sigma$ and $2 \sigma$ range around the background. . . . 4

1.2 Crossections for the Higgs production at LHC as function of the Higgs mass $m_{H}$ [4]. . . . . . . . . . . . . . . . . . . 5

1.3 Branching ratios for the different decay channels of the Higgs boson [4]. 6

1.4 Transverse view of the CMS detector. . . . . . . . . . . 8

1.5 General layout of the CMS silicon microstrip tracker. The single-sided modules are marked in red, the stereo modules in blue. . . . . . . . 10

1.6 a: Number of points collected by tracks as a function of rapidity. Pixels are not included and stereo points are counted once. b: Material budget excluding pixels and material outside the tracking volume. [15] 11

1.7 a) Working principle of a silicon particle detector. b) Schematic structure of the CMS silicon strip sensors . . . . . . . . . . . . . . 12

1.8 Silicon microstrip modules for the inner barrel and the endcap. . . . . 14

1.9 Exploded 3D-View of an endcap tracker module. . . . . . . . . . . . . 14

1.10 Mechanical support structure of the Tracker Outer barrel (TOB). . . 15

1.11 Left: 3D detail of the mechanical support structure of the inner barrel (TIB). The modules are supported on carbon fiber half cylinders which have openings to save weight and route the services to the modules. Right: Endcap disk with the petal layout. The wedge-shaped sensors are mounted on the petals. . . . . . . . . . . . . . 16

1.12 Endcap petal supporting the modules. . . . . . . . . . . . . . 17

1.13 The CMS trigger is organized into several levels. Level 1 is a hardware and the higher level triggers (HLT) are software triggers. For the HLT data of reconstructed tracks is used. This makes it necessary to do part of the track reconstruction online. . . . . . . . . . . . . 18

1.14 Sketch of the working principle of the alignment system. The laser beam passes through several silicon layers, allowing to measure the displacement of each layer. Beam tilts can be separated from individual layer movements. . . . . . . . . . . . . . . . . . . 20

2.1 Setup for measuring the laser stability . . . . . . . . . . . . 23

2.2 a) Electrical pulse at the end of the fromt-end amplifier from a laser pulse signal. b) Laser profile measured by a silicon microstrip detector. 24 
2.3 The distributions of the height ( $\propto$ laser intensity) and the $\sigma$ of the gaussians fitted to the laser pulses. . . . . . . . . . . . . . 24

2.4 The distributions of the fitted laser spot. . . . . . . . . . . . 25

2.5 Section of a silicon strip sensor. Except for the aluminium, all parts are transparent for infrared light. . . . . . . . . . . . . 26

2.6 Silicon strip sensor with a hole etched into the backplane metallization 27

2.7 Absorption coefficient in silicon after [23]. . . . . . . . . . . . . 28

2.8 Setup for the measurement of the light transmission through a silicon sensor. The Nd-YAG laser operates in pulsed mode, and the $1080 \mathrm{~nm}$ diode in cw mode. The beam profile is measured with a CCD chip in the beam profiler. . . . . . . . . . . . . . . . . . . . . 29

2.9 Beam profiles measured directly and after passing through a silicon layer. The axes in the horizontal plane give the position in $\mathrm{mm}$, the vertical axis the CCD response in ADC counts. The surface formed by the shades is the measurement, the superimposed grid a two-dimensinal gaussian which was fitted to the data. As transmission coefficient we take the ratio of the volumes under the gaussians, which are proportional to $h \cdot \sigma^{2} \ldots \ldots \ldots \ldots$

2.10 The beams reflected at the surfaces may interfere. This will modulate the reflected, transmitted and absorbed intensities. . . . . . . . . 31

2.11 Beam profiles measured with a strip detector. The left profile is distorted due to interference effects. In the right plot the signal of a single strip is plotted while the laser beam is scanned across it. The height of the gaussian is a measure of the signal response of that strip to the laser light. . . . . . . . . . . . . . . . . . . 32

2.12 Signal response of the detector strips when a laser is scanned across the sensor. The modulation is an interference effect. The strips with zero response are bad channels. . . . . . . . . . . . . . 33

2.13 Interference pattern before and after etching a hole into the backplane. 34

2.14 Interference patterns observed on various wafers. Dark regions correspond to absorption maxima, light regions to absorption minima. . . 35

2.15 Interference pattern as measured on a strip detector, and the thickness change $\Delta y$ inferred hereof. Note that the sign of $\Delta y$ is ambiguous and has been arbitrarily chosen to be positive in this plot. . . . . . 37

2.16 Measured accuracy for finding the laser spot as a function of $\mathrm{S} / \mathrm{N}$ ratio. The laser spot was kept at the same position, while the intesity was changed. The solid curve is the calculated precision, the dashed curve is a hyperbola fitted to the results of the gaussian fit. . . . . . 38

2.17 Simulation of the reconstruction of the laser spot position. The accuracy $\Delta m$ and the laser width $\sigma_{L}$ are given in pitch units. Results for a gaussian fit and the center of gravity algorithm are shown. The curve is the calculated precision (eq.2.12). . . . . . . . . . 39

2.18 Comparison of a single pulse with $\mathrm{S} / \mathrm{N}=1$ and the average over 500 pulses. . . . . . . . . . . . . . . . . 40 
2.19 Effective signal to noise ratio versus the number of sampled pulses. The curve follows only in the beginning the expected square root function. . . . . . . . . . . . . . . . . . . . .

2.20 Setup used for determining the position accuracy of the laser spot. The support of the collimator can be tilted and the first detector can be moved with a precision translation stage. . . . . . . . . . . .

2.21 Left: Laser spot position plotted against the precision stage position for one scan. Right: Distribution of the slopes of the fitted straights for several scans.

2.22 Left: Residuals measured on a wafer as function of the position. Light and dark areas correspond to $+50 \mu \mathrm{m}$ and $-50 \mu \mathrm{m}$. The observed structures are similar to the interference patterns. Top right: Distribution of the residuals on the whole wafer. Bottom right: Distribution of the residuals in upper left and lower right quadrant. . . . . . . . . 43

2.23 Residuals measured in the same sensor region before and after the hole in the backplane was etched.

2.24 Residuals measured on the second layer while the first one is displaced. The pitch of the sensors is $50 \mu \mathrm{m}$.

2.25 The beam can be tilted with a precision screw. $\Delta m$ is the screw setting. The plot on the left shows the measured laser spot positions as function of the screw setting. The histogram on the right shows the distributions of the residuals on both modules. . . . . . . . . . . . 46

2.26 Measurement in which a displacement was combined with the beam tilt. The curves on the left are the randomly generated settings while the symbols are the measured positions. On the right the histograms with the residual distributions are shown. . . . . . . . . . . . . . 47

3.1 Layout of the laser alignment system. The arrows indicate the positions where the fibers run radially inwards. . . . . . . . . . . .

3.2 Refraction is considered to be an uncertainty and is treated similar to multiple scattering of a particle track. . . . . . . . . . . . . 51

3.3 Parameters describing the disk translations along the $\mathrm{x}$-axis. The sum of the individual movements $\Delta x_{k}$ is 0 as well as their average slope. . . . . . . . . . . . . . . . . . 56

3.4 Distributions of the residuals for the individual disk displacements and for the collective movements. A ring of 8 beams at radius $R_{0}=564 \mathrm{~mm}$ was used. . . . . . . . . . . . . . . . . . 62

3.5 Precision of the reconstructed parameters as a function of the number of beams. The beams are at a radius $R_{0}=564 \mathrm{~mm}$ and are equally

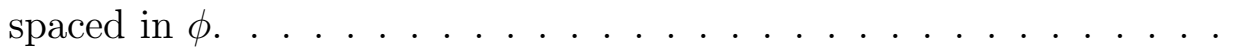

3.6 Precision of the reconstructed parameters as a function of the accuracy $\Delta m$ with which the intersection point of laser beams and disks is found. There are 8 beams which are equally spaced in $\phi$. The results do not depend on $R_{0} \ldots \ldots \ldots \ldots$. . . . . . . . . . . . 6 


\section{List of Tables}

2.1 Transmission of light through a silicon layer of $300 \mu \mathrm{m}$ thickness and $R_{\text {strip }}=0.25$. $R_{\text {strip }}$ has the same value for the modules of the final CMS microstrip sensors. . . . . . . . . . . . . . . . . 29

2.2 Transmission through $300 \mu \mathrm{m}$ thick sensors measured for a laser diode with $\lambda=1080 \mathrm{~nm}$ and a NdYAG laser with $\lambda=1064 \mathrm{~nm}$. The transmission was measured at several different locations on the sensors. . . 31

$3.1 \phi$-position of the 8 laser beams of ray $4 \ldots \ldots \ldots$

3.2 Expected accuracy for finding the position of the laser spot on the modules used in the laser alignment system. A S/N-ratio of 10 was assumed and the values are given for $\sigma_{L}=0.25 \mathrm{~mm}$ and $\sigma_{L}=1 \mathrm{~mm}$. . 52

3.3 Summary of all contributions to the overall precision with which the intersecting point of laser beams and disks can be found for the internal endcap alignment. The mechanical accuracies are estimates. . . 54

3.4 Calculated precisions for the reconstruction of the parameters for the different scenarios. All values are in $\mu \mathrm{m} \ldots \ldots 61$ 


\section{Lebenslauf}

Name:

Geboren:

Staatsangehörigkeit:

September 1974:

Juni 1986:

Wintersemester 1986:

Sommersemester 1994:

Oktober 1995:

Nov. 1995-Nov. 1996:

Dezember 1996:
Bruno Wittmer

26. September 1968 in Lissabon, Portugal

Deutsch / Portugiesisch

Einschulung in die Deutsch Schule Lissabon

Allgemeine Deutsche Hochschulreife an der Deutschen Schule Lissabon

Beginn des Studiums der Physik an der Universität Stuttgart

Wechsel an die Rheinisch-Westfälische Technische Hochschule Aachen und Beginn der Diplomarbeit am I. Physikalischen Institut

Abschluß als Diplom-Physiker an der RWTH Aachen.

Zivildienst in Aachen

Beginn der Promotion am I. Physikalischen Institut der RWTH Aachen 